\title{
Innovative Game-Aided Rehabilitation Platform for Rehabilitation of Balance in Children with Cerebral Palsy
}

\author{
By
}

Kavisha Mehta

A Thesis submitted to Faculty of Graduate Studies of

The University of Manitoba

In the partial fulfillment of the requirements of the degree of

\section{MASTER OF SCIENCE}

\author{
College of Rehabilitation Sciences \\ University of Manitoba \\ Winnipeg
}

Copyright (C 2021 by Kavisha Mehta 


\section{ABSTRACT}

Introduction: Cerebral Palsy (CP) is one of the leading causes of disability globally. Children with CP often develop imbalance and gait abnormality resulting in mobility limitations. Early intervention with intensive, repetitive, task-oriented practice is the key to functional independence. However, often these intensive programs lack motivation and engagement that results in low adherence and discontinuation of physiotherapy. To address this gap, an innovative, computerized game-aided rehabilitation (CGR) platform is designed for dual-task balance training. This platform provides an integrated approach to assess and treat the balance and executive cognitive functions in young children with CP.

Methodology: For the known-group validity, 50 participants (25 TD and 25 CP affected children) were recruited. Comparisons were made between the two groups for DTI and task performances to evaluate the group differences. For the pilot intervention study, 16 children with $\mathrm{CP}$ were recruited who were randomized into two groups viz. Conventional balance training group (CBG) and Experimental group (XG). Both group received their respective therapy thrice a week for 12 weeks. The primary outcome measures were (Peabody Developmental Motor Scale-2, Gross Motor Function Classification-88, and Pediatric Balance Scale) and CGR assessment measures [COP Analysis, Visuo-Motor (VM) tracking, and Cognitive Game (CG)]. Semi-structured interview were conducted for XG participant's parents to explore their views regarding the use of CGR. 
Results: The TD group was engaged in DT whereas the CP group was struggling with balance control. For the intervention study, participants in the XG demonstrated greater improvements in all clinical and CGR outcome measures whereas the CBG demonstrated a decline in VM tracking a CG performance post-intervention. The XG parents conveyed their children's problems, their expectations, their challenges, and the positive effects of CGR balance training.

Conclusion: The CGR assessment was able to differentiate between the TD and CP children's DT functional performance. The CGR balance training was found to be feasible and engaging for balance training in children with CP. The substantial improvement of balance and gross motor functions in the XG more than the CBG illustrates the effectiveness of the CGR balance training and provides baseline evidence for commencing a full-scale RCT. 


\section{ACKNOWLEDGEMENT}

Firstly, I would like to thank my advisor Dr. Tony Szturm for his ample guidance and feedback towards the completion of my thesis work. I will always be appreciative of all his positive criticism that encouraged me to be the best version of myself. I am sincerely thankful to my internal advisor Dr. Sanjay Parmar and external advisor Dr. Rasit Eskicioglu for their expert advice, inputs, support, and attention to detail throughout this process. My sincere thanks to Dr. Ruth Barclay for her guidance and support during the initial stages of my thesis formulation. My special thanks to Anuprita Kanitkar and Vedant Sakhalkar for giving a kick start to my journey in Canada and being my support pillars here. I am appreciative of all the efforts put in by the staff, faculty, students, and colleagues at SDM College of Physiotherapy for all their help and cooperation during the process. Without them, this would have been impossible to accomplish. I want to mention a special thanks to Deepthi Shetty, for being patient and cooperative in different phases of this study. I cannot thank enough to all the study participants and their parents for all their patience and co-operation during the study period. I am grateful for all the support extended by Susan Vanstone during my M.Sc. program and for helping me navigate throughout my journey at the College. I would like to extend my sincere thanks to Dr. Robert Tate for always being understanding and supportive through this journey. I am grateful to the College of Rehabilitation Science, The University of Manitoba for the Jal Tata Award 2019. I cannot conclude this acknowledgment without thanking my family members for their constant support and blessings with all the choices that I made throughout my journey. They have always encouraged me to follow my passion without any hesitance. 


\section{DEDICATION}

I would like to dedicate this thesis to my beloved parents, Mr. Manoj Gunvantrai Mehta and Mrs. Rita Manoj Mehta, without whom I would not be here today. Whole-hearted thanks to my parents for being my strength and inspiration throughout the journey of my life. In addition, this journey would have been impossible without my sister and brother-in-law, Mrs. Pooja Nirav Desai, and Mr. Nirav Ashok Desai, whose endless support and admiration always motivated me. I would also like to show my gratitude to my mentor, Dr. Prabhakar Devanpalli for the constant support and encouragement in this accomplishment. Moreover, this work would have been incomplete without the support and motivation from a very special person, Mr. Akash Pinakin Shah, without whom I would have not been able to overcome the difficulties in the process of completing my thesis. It would be less to mention heartfelt thanks for all the laughter and joy you brought to the difficult times in this journey. The efforts and motivation paid off positively through this work.

Kavisha Mehta. 


\section{Table of Contents}

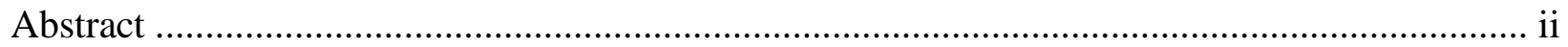

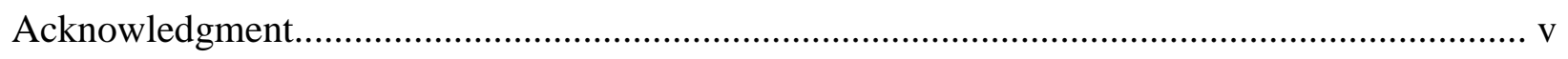

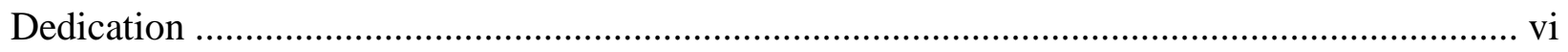

Table of Contents .........................................................................................................

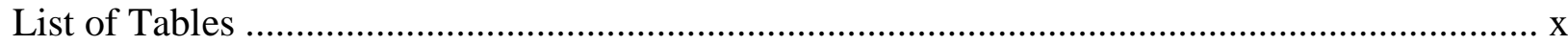

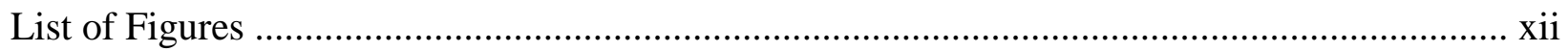

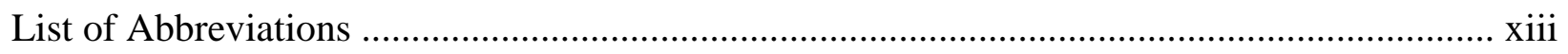

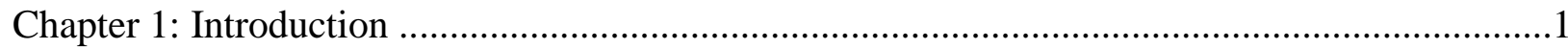

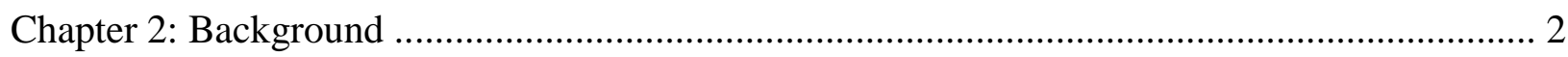

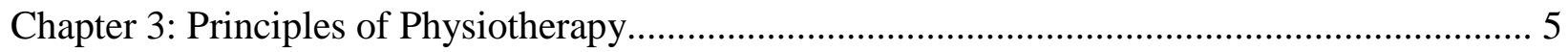

3.1 Task-Specific, Repetitive, Goal-oriented Training...................................................... 5

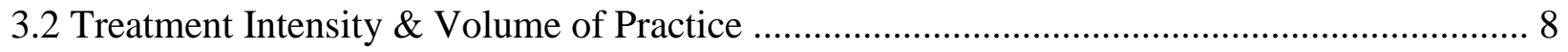

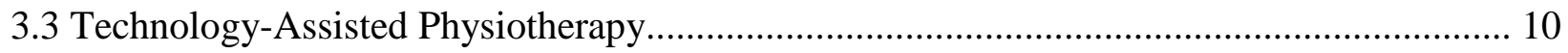

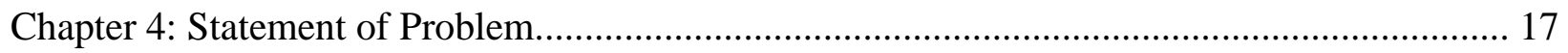

Chapter 5: Development of Computerized Game-aided Rehabilitation (CGR) Platform........... 19

Chapter 6: Purpose \& Objectives of the Study................................................................... 22

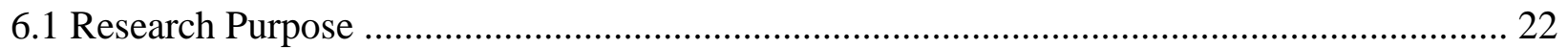

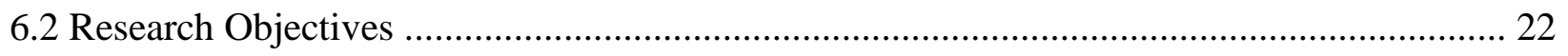

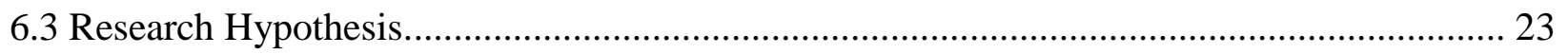

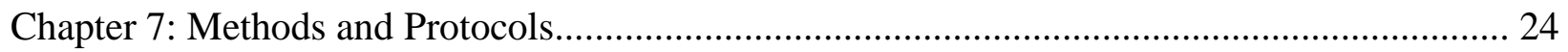

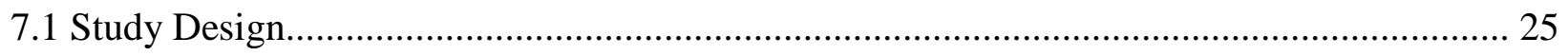

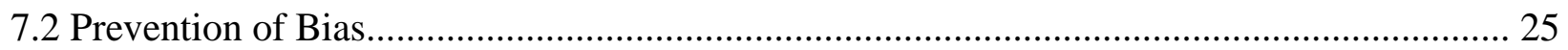


7.3 Sample Size.......

7.4 Ethics Approval \& Informed Consent................................................................... 25

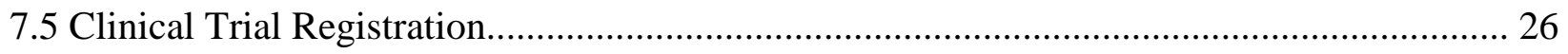

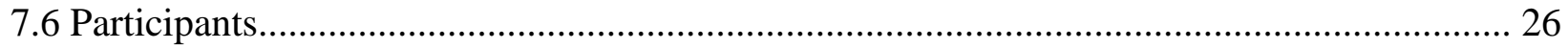

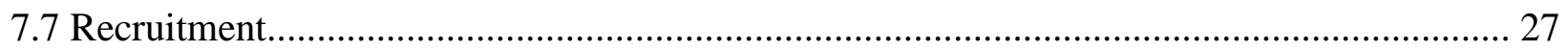

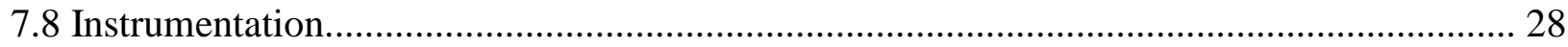




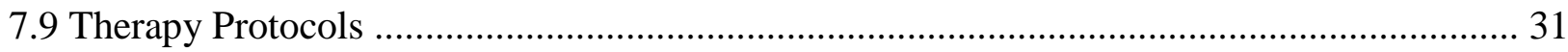

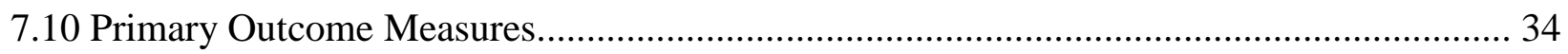

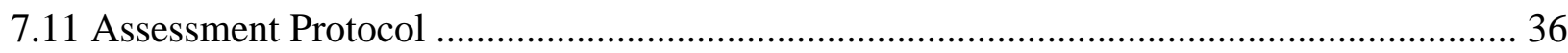

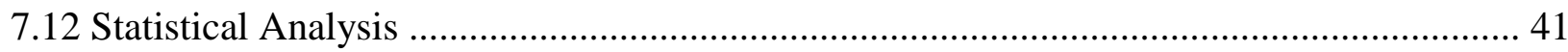

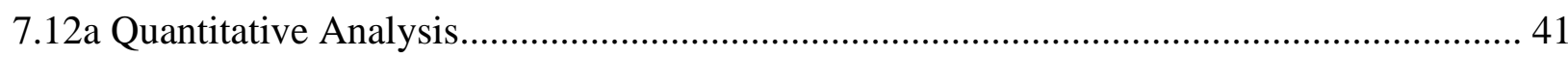

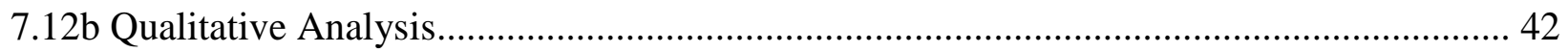

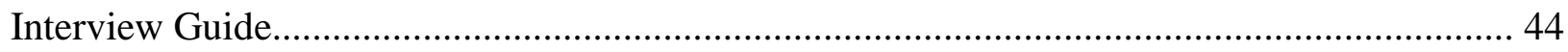

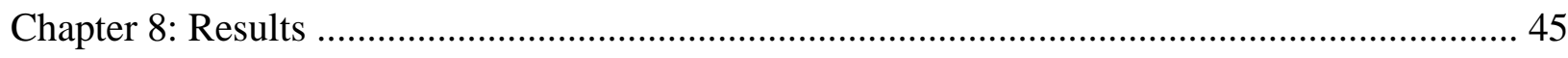

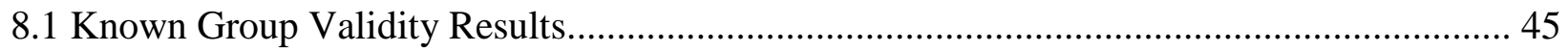

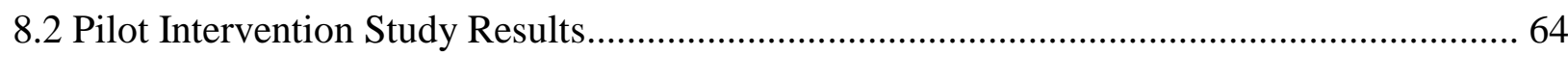

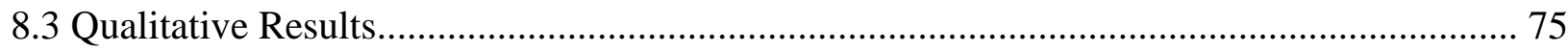

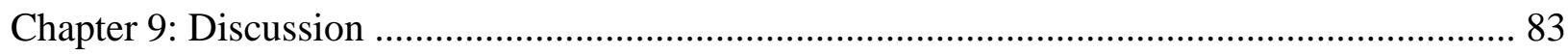

9.1 Known-Group Validity................................................................................................... 83

9.2 Feasibility of CGR platform for Balance Rehabilitation of children with CP....................... 85

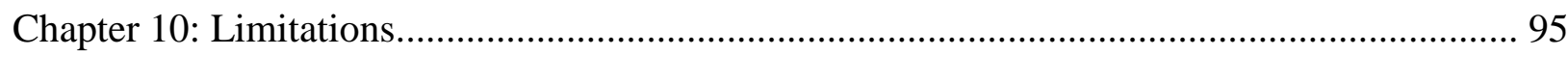

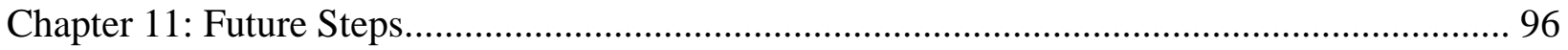

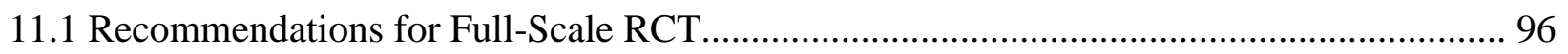

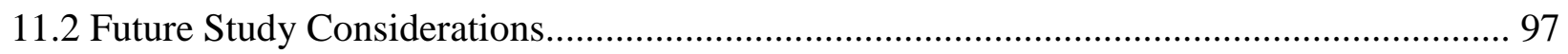

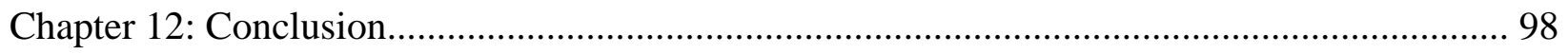

References ……… 


\section{$\underline{\text { List of Tables }}$}

Tables 1: Demographic data for the Known-Group Validity Study.........................45

Table 2: Results of Unpaired T-test for Total Path Length (TPL) .......................46

Table 3: Results of Unpaired T-test for Peak to Peak COP Excursion in Medio-Lateral direction

(P2P_ML)

Table 4: Results of Unpaired T-test for Peak to Peak COP Excursion in Antero-Posterior

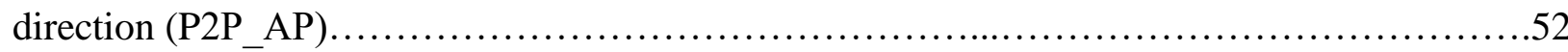

Table 5: Engagement Frequency Analysis for Visuo-Motor Tracking Task..................56

Table 6: Results of Unpaired T-test of Success Rate (SR) for Cognitive Game Tasks..........57

Table 7: Results of Unpaired T-test of Movement Error (ME) for Cognitive Game Tasks.......57

Table 8: Results of Paired Samples T-test for Comparison of task Differences in TD group....60

Table 9: Results of Paired Samples T-test for Comparison of task Differences in CP group ....61

Table 10: Demographic Table for the experimental \& Control group participants for the pilot intervention study

Table 11: Results of Clinical Outcome Measures pre and post-intervention...................64

Table 12: Table for Pre-Post changes of Total Path Length (TPL) on Fixed Surface...........67

Table 13: Table for Pre-Post changes of Total Path Length (TPL) on Compliant Surface.

Table 14: Table for Pre-Post changes of Average Residual Error (ARE) for Visuo-Motor

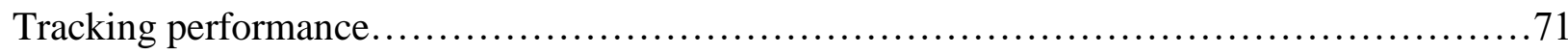

Table 15: Results for Pre-Post changes of Success Rate for Cognitive Target Game............72

Table 16: Results for Pre-Post changes of Movement Error for Cognitive Target Game ........74 
Table 17: Theme 1 - Problem List.............................................. 75

Table 18: Theme 2 - Expectations .....................................................

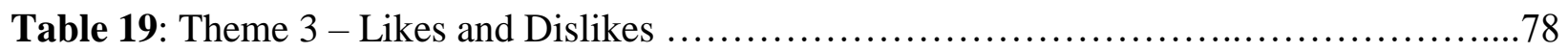

Table 20: Theme 4 - Choices of Games ............................................. 79

Table 21: Theme 5 - Positive Effects of Experimental Intervention .........................80

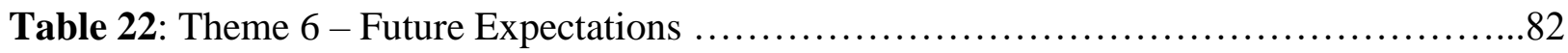




\section{List of Figures}

Figure 1: Bar chart for the Total path length for all tasks performed on Sponge Surface.......47

Figure 2: Bar chart for the Total path length for all tasks performed on Fixed Surface..........48

Figure 3: Bar chart for the ML P2P COP Excursion for all tasks performed on Sponge

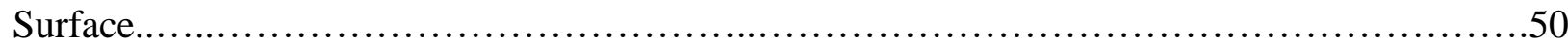

Figure 4: Bar chart for the ML P2P COP Excursion for all tasks performed on Fixed

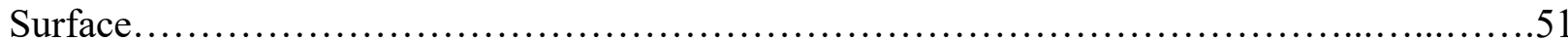

Figure 5: Bar chart for the AP P2P COP Excursion for all tasks performed on Sponge

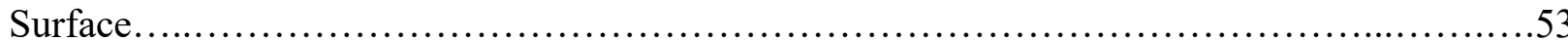

Figure 6: Bar chart for the AP P2P COP Excursion for all tasks performed on Fixed

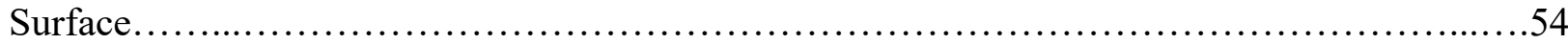

Figures 7: Plots of VM Tracking performance engagement Levels.......................55

Figure 8: Bar Chart for the Success Rate for all game tasks performed on Fixed \& Sponge

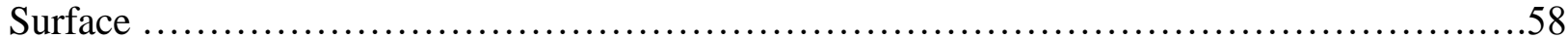

Figure 9: Bar Chart for the Movement Error for all game tasks performed on Fixed \& Sponge

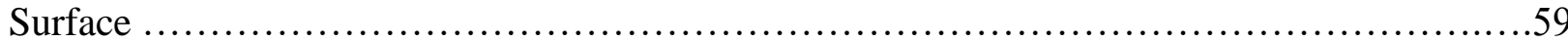




\section{List of Abbreviations:}

CGR - Computerized Game-aided Rehabilitation (Page No. 1)

CP - Cerebral Palsy (Page No. 2)

ABI - Acquired Brain Injury (Page No. 27)

RCT - Randomized Controlled Trial (Page No. 1)

TD - Typically Developing (Page No. 1)

XG - Experimental Group (Page No. 24)

CBG - Conventional Balance Training Group (Page No. 24)

NDD - Neuro-Developmental Disorders (Page No. 2)

RTP - Repetitive Task Practice (Page No. 1)

HABIT - Hand-Arm Bimanual Intensive Therapy (Page No. 3)

CIMT - Constraint-Induced Movement Therapy (Page No. 3)

RT - Reaction Time (Page No. 5)

MT - Movement Time (Page No. 5)

GMFCS - Gross Motor Functional Classification System (Page No. 27)

GMFM-88 - Gross Motor Function Measure-88 (Page No. 35)

TUG - Timed Up and Go Test (Page No. 6)

RM - Repeated Measure (Page No. 8)

COM - Center of Mass (Page No. 11)

FSST - Four Step Squared Test (Page No. 12)

RMS - Root Mean Square (Page No. 12)

PC - Personal Computer (Page No. 12)

COP - Center of Foot Pressure (Page No. 13)

AP - Antero-Posterior (Page No. 13)

BBS - Berg Balance Scale (Page No. 13)

TEE - Total Energy Expenditure (Page No. 13)

MET - Metabolic Equivalent (Page No. 13) 
DT - Dual-Task(ing) (Page No. 19)

USB - Universal Serial Bus (Page No. 19)

FSA - Force Sensor Array (Page No. 20)

DTI - Dual-Task Interference (Page No. 22)

HREB - Health Research Ethics Board (Page No. 25)

S.D.M. - Shree Dharmasthala Manjunatheshwar (Page No. 25)

OPD - Out Patient Department (Page No. 27)

MAS - Modified Ashworth Scale (Page No. 27)

PDMS-2 - Peabody Developmental Motor Scale- Second Edition (Page No. 28)

PBS - Pediatric Balance Scale (Page No. 28)

NFER - Neuro-Function Evaluation Research (Page No. 28)

SPSS - Statistical Package of Social Sciences (Page No. 28)

LOS - Limit of Stability (Page No. 21)

ADL - Activities of Daily Living (Page No. 35)

BOS - Base of Support (Page No. 35)

TPL - Total Sway Path Length (Page No. 35)

ML - Medio-Lateral (Page No. 35)

mCTSIB - Modified Clinical Test of Sensory Integration in Balance (Page No. 36)

C1, C2, ...., C8 Control Group Participants (Page No. 62)

$\mathrm{X} 1, \mathrm{X} 2, \ldots, \mathrm{X} 8$ - Experimental Group Participants (Page No. 62)

OM - Outcome Measure (Page No. 64)

EO - Eyes Open (Page No. 36)

EC - Eyes Closed (Page No. 36)

VM - Visuo-Motor Tracking (Page No. 36)

CG - Cognitive Game (Page No. 36)

CG1- Easy Cognitive Game Target (Page No. 36)

CG2- Difficult Cognitive Game Target (Page No. 36)

_F - On Fixed surface (Page No. 46) 
_C - On Compliant surface (Page No. 46)

SR - Success Rate (Page No. 39)

ME - Movement Error (Page No. 39)

ARE - Average Residual Error (Page No. 37)

F - Female (Page No. 45)

M - Male (Page No. 45)

SEM - Standard Error Measure (Page No. 45)

Diff - Difference (Page No. 64) 


\section{Chapter 1: Introduction:}

The present pilot study project focuses on the evaluation of an innovative, engaging Computerized Game-Aided Rehabilitation (CGR) platform for use in the rehabilitation of balance in young children with Cerebral Palsy $(\mathrm{CP})$. The main purpose of this project is to determine the acceptance, accessibility, and feasibility of using the CGR platform for balance rehabilitation in children with CP. The final purpose is to develop evidence for future full-scale randomized control trial $(\mathrm{RCT})$.

This pilot project will focus on developing known group validity of the CGR platform by comparing the performance of typically developing (TD) children with children affected with $\mathrm{CP}$. The intervention delivery by the means of the CGR platform for balance rehabilitation in the form of this pilot project will help in the examination of the acceptability and compliance of the participants to the CGR program. This study will estimate the treatment effect of repetitive task practice (RTP), game-aided exercise program on dynamic balance skills (i.e. transitions to standing, turning, stepping, and locomotion) in children diagnosed with CP. The preliminary estimation of treatment effects will be determined based on the pre and post-intervention performance scores of the primary outcome measures of this study. This will help us determine if any required adaptations for the assessment or intervention protocols before proceeding with a full-scale RCT. 


\section{Chapter 2: Background:}

Pediatric physical therapists frequently encounter children diagnosed with neurodevelopmental disorders (NDD) such as Cerebral Palsy (CP). Rosenbaum et. al., (2007) defines "Cerebral Palsy (CP) as a group of permanent disorder of the development of movement and posture, causing activity limitation that is attributed to non-progressive disturbances that occurred in the developing fetal or infantile brain. The motor disorders of CP are often accompanied by disturbance of sensation, perception, cognition, communication, and behavior, by epilepsy, and by secondary musculoskeletal problems". CP affects a large number of children and is the leading cause of disability worldwide. (Stavsky et al., 2017, Robertson et al., 2017, Braun et al., 2016) The overall global prevalence of CP is 2 per 1000 live births. (Gulati et al., 2018) Substantial balance impairments are common (Rosenbaum et al., 2006) and this results in limitations in mobility function necessary for activities of daily living and participation in social events such as in school, leisure, and play activities. Weight-bearing and dynamic balance control in standing is an important function and is critical for bone and muscle development.

There are many aspects to consider when developing a therapy program intended to improve balance, optimize the neurodevelopment in children with neurodevelopmental disorders, improve locomotion function, and thus prevent the development of secondary disabilities and non-use sequelae (like spasticity, contractures, muscle wasting, disuse atrophy, etc.).

(Rosenbaum et al., 2006)

According to the current literature, various studies focus on upper limb function rehabilitation but very few studies are available in the literature that focuses primarily on balance rehabilitation in younger children with $\mathrm{CP}$. Children with $\mathrm{CP}$ undoubtedly benefit from intensive, 
repetitive, well-resourced therapy protocols. For example; consider the various forms of repetitive task practice (RTP) such as Hand Arm Bimanual Intensive Therapy (HABIT) or Constraint-Induced Movement therapy (CIMT), a guided and intense exercise program with a focus on hand and arm activities that do produce significant improvements in arm function. (Cao et al., 2015, Wolf et al., 2006, Liepert, 2006, Tarkka et al., 2005, Rijntjes et al., 2005, Martin et al., 2008, and Taub et al., 2004)

When rehabilitation regimens are prescribed for these children, which incorporates RTP; adherence is often low because exercises are tedious and uninteresting to them. Therefore, it is difficult to sustain motivation in intensive RTP to achieve long-term rehabilitation goals. If one is to expect good long-term outcomes for these children with physical and mental disabilities then the training needs to be flexible, motivating, engaging, progressive, and of large volume. (Barreca et al., 2007) Parents and clinicians rate motivation as the most influential personal characteristic to determine motor and functional outcomes in these children. (Malhi \& Singhi, 2002)

With the evidence of improvement in upper extremity function with RTP, we propose to perform an exercise regimen with RTP for balance reaction activation and balance function restoration. The proposed regimen involves game-assisted therapy to address the engagement of these children in long-term therapy involving RTP. The main goal of this research project is to develop a platform for the provision of high-quality rehabilitation services that are cost-effective and engaging for young children with CP. Such a platform will ensure better adherence to longterm rehabilitation approaches.

The current literature provides evidence that task-specific training best facilitates motor development and early physical therapy/rehabilitation intervention is critical to aid this purpose 
in therapy. (Yang et al., 2013) After the deliberate review and analysis of existing literature, Novak et al., (2017) concluded that the early diagnosis for children with CP is most crucial for the better outcome of the therapy. (Novak et al., 2017) The intervention started early in life positively influences the neuro-motor development of children with neurodevelopmental disorders (i.e. intervening before the development of gait abnormalities and other deficits will prevent any deconditioning of the neuro-musculoskeletal system). (Novak et al., 2017) This concept is also reinforced by Reid et al., (2015) that the initial years of life are most crucial for intervention to improve the outcomes of the therapy. (Reid et al., 2015) The primary focus of their review was to summarize the factors that influence the outcomes of therapy. Most of the therapeutic approaches in the current literature were based on the activities that involved the child and their behavior. The most influential factors that yield the best outcomes of the therapy are intensity, frequency, transfer of training to activities of daily living, and the suitability of the treatment to age-appropriate goals. (Reid et al., 2015) This information urges me to develop detailed evidence and a theoretical basis on these important principles of physiotherapy. 


\section{Chapter 3: Principles of Physiotherapy:}

The most important principles of physiotherapy included in this project are detailed below: (Wilkins et al., 2017, Harvey, 2009)

\subsection{Task-specific, Repetitive, Goal-oriented Training:}

Task-specific training is defined as therapy or interventions that are focused on contextspecific tasks. (Hubbard et al., 2009, Teasell et al., 2008, Toovey et al., 2017) The involvement of practicing real-life tasks like walking, writing, etc. to master a specific skill is defined as taskoriented training. For instance, a treadmill training intervention for gait training is superior to any other form of gait training used for rehabilitation. (Harvey 2009, Willoughby et al., 2009, Sullivan et al., 2007) Researchers prove that task-specific training facilitates motor learning and helps in the retention of the motor memory to execute particular tasks during the functional performance of the activities. (Wilkins et al., 2017, Maier et al., 2019, Boyd et al., 2010) A study performed by Boyd et al.(2010) examined the recovery of fine motor functions in 18 participants who suffered a stroke. The control group participants received non-specific general arm exercises like pushing a beanbag, stacking cones, erasing, etc. On the other hand, the experimental group was trained for the serial tasks using a joystick. Both groups were assessed while performing a serial task of holding a joystick and moving it to targets positioned at several points in space. On average, both groups performed 400 repetitions of their respective training movements for 3 days. There was a significant improvement noted in the mean reaction time (RT) and the movement time (MT) in the task-specific group as compared to the general increased practice group. (Boyd et al., 2010) Thus, we can state here that if an improved balance 
is an expected outcome, training of tasks involving the facilitation of balance reactions would be the best intervention.

Salen \& Godwin,(2009) conducted a study to evaluate the effectiveness of task-oriented training to improve mobility functions in children with $\mathrm{CP}$. They recruited 10 children with $\mathrm{CP}$ in the age group of $4-11$ years and GMFCS Level 1-3 and randomized them to control $(n=5)$ and experimental $(\mathrm{n}=5)$ groups. Both groups received two sessions per week for 5 weeks and they were assessed at baseline and post 5 weeks. The control group received conventional PT for walking and balance training without a direct focus on functional task training. The experimental protocol comprised task-specific training including sit to stand; walking up and down the ramp and stairs, overground walking in forward, backward, and left and right directions, standing balance activities including reach outs beyond the arm length, weight shifts in all directions, and activities like kicking a ball. The results of the standing subtest of GMFM- 88 demonstrated an $8.2 \%$ improvement in the experimental group as opposed to $3.8 \%$ in the control group. The walking scores of GMFM- 88 were improved by $9.0 \%$ in the experimental group whereas only a $3.8 \%$ increase was observed for the control group. The results also demonstrated a reduced time taken to complete Timed-Up \& Go Test (TUG), which was an 11.2\% decrease in the experimental group as compared to a 3.7\% decrease in the control group after 5 weeks of respective interventions. (Salem \& Godwin, 2009)

Kumban et. al. (2013), examined the effects of task-specific training on the performance of sit-to-stand in children with mild to moderate CP (GMFCS Level 1-3) aged 6-15 years. The 21 study participants were randomly allocated to the control arm $(n=12)$ or the experimental arm $(\mathrm{n}=11)$. Both groups' participants were receiving routine physiotherapy sessions including stretching, strengthening, balance training, and locomotion training. The experimental program 
focused on specific training for the sit-to-stand activity. For instance, the participants were individually trained for symmetrical weight shifting, forward-leaning, buttock lifting, and standing up. They were also provided a specific goal like reaching forward for leaning forward, etc. The experimental group demonstrated a $21.8 \%$ improvement in Five Times Sit-to-Stand Test results as opposed to only $6.7 \%$ improvement in the control arm post- 6 weeks of respective intervention. This evidently proved that task-specific training is beneficial for training the tasks of functional importance in these children for fruitful results of the therapeutic interventions. (Kumban et al., 2013)

Goal-oriented training, on the other hand, is defined as an intervention with a focused goal. An activity-based approach (Mastos et al., 2016) involving the active participation of participants in the accomplishment of task-specific goals to improve functional performance. Researchers have proven that the learning and performance of motor skills are influenced by the knowledge of the effects of movement. (Maier et al., 2019 and Wulf \& Prinz, 2001) For instance, a goal given to touch an attractive ball while reaching out during balance training would yield better participation of a child as compared to simply asking a child to reach in a particular direction. (Kumban et al., 2013) Or a child would perform better if he/she were made to play a game with a specific target to achieve while using the entire range of joint motion rather than just performing a non-specific range of motion exercise.

As derived from the goal-setting theory by Locke \& Latham, (2002), a goal must be specific to individual needs and at the same time must be difficult enough to push the limits of comfort to expand the room for improvement. This will ensure better performance of an individual, which will steer to the personal satisfaction of the participant and influence their commitment to long-term efforts. (Locke \& Latham, 2002) The other factors that influence the 
goal-setting would include the complexity of the task, self-efficacy of the participant, commitment to continued efforts, and the feedback one receives. (Locke \& Latham, 2002 and Bandura et al., 2001) We must keep in mind these positive influences of the goals while planning a therapeutic approach to keep children engaged and motivated in therapy for the long term and developing functional independence in those participants. Many studies have reported this principle to be one of the most influential while considering the therapeutic benefits of an intervention. (Harvey, 2009, Maier et al., 2019, Locke \& Latham, 2002, Bandura et al., 2001, and Østensjø et al., 2008)

\section{2: Treatment Intensity \& Volume of Practice:}

The effectiveness of the rehabilitation protocol is proportional to the intensity and volume of training and the task-specificity of the exercise program (Bottos et al., 2001, Jetelaar et al., 2001, Trahan \& Malouin, 2002, and Odman \& Oberg, 2006). The treatment intensity is defined as the actual cost of exercise (Peungsuwan et al., 2017). The intensity could be expressed as an absolute value, which is the actual rate of energy expenditure (Qi et al., 2018), or a relative value that is defined as the energy cost of an exercise relative to an individual's maximal capacity (Peungsuwan et al., 2017 and Qi et al., 2018). For instance, the intensity of the strength training could be decided by examining the 1 RM (Repeated Measure) or 10 RM. (Surana et al., 2019). The maximal heart rate could also be a deciding factor for the intensity of cardio-respiratory exercises; intensity for other forms of exercises can be varied by changing the speed like walking at a faster pace or running. (Peungsuwan et al., 2017) These measures are used as intensity variables for strength and endurance training. However, there is limited evidence that examines the intensity of neuro-motor exercises. (Peungsuwan et al., 2017, Nelson et al., 2007, and JAGS, 
2001). The volume of practice, on the other hand, is defined as the actual amount of time spent on the particular type of exercise and the number of sessions received in a certain amount of time. (Hsu et al., 2010) It takes into consideration the duration and frequency of intervention. (Power \& Clifford, 2013) It is an important factor to consider while implementing an intervention. When considering these factors, adherence is an important aspect that influences the effectiveness of an intervention. It is reported that adherence is often influenced by the intensity and the volume of intervention implemented. (Peungsuwan et al., 2017) Thus, one must keep in mind all these factors while prescribing an intervention to ensure an acceptable amount of adherence to the intervention.

Various other studies have proven that task-specific training yields the best results in the expected functional outcomes. (Locke \& Latham, 2002, Bandura et al., 2001, and Østensjø et al., 2008) At the same time, repetition is the key to improvement in function that will reinforce betterment in the performance of the real-life functional tasks. (Cao et al., 2015, Wolf et al., 2006, Liepert, 2006, Tarkka et al., 2005, Rijntjes et al., 2005, Martin et al., 2008, and Taub et al., 2004)

This brings us to the conclusion that task-specificity with goal orientation and repetitive practice are important components that must be given priority while developing a novel therapeutic approach.

Varieties of approaches are used to incorporate these important principles of physiotherapy in practical/clinical use while integrating motivation to reinforce adherence to therapy for rehabilitation in children affected with $\mathrm{CP}$. They are detailed below: 


\subsection{Technology-Assisted Physiotherapy:}

As noted in the previous section, intensity is one of the factors affecting adherence to a longterm commitment to exercises. (Williams, 2008) Engagement and motivation to perform these task-specific repetitive practices regularly is another important factor that influences adherence to the intervention. (Peungsuwan et al., 2017) When developing a protocol requiring long-term compliance to therapy, one of the downsides faced is lack of interest and motivation in most young children with neuro-motor impairments. There is a need for innovation to incorporate engagement in therapy. Digital media, in particular, computer games have received considerable interest as a therapeutic approach from researchers and clinicians across the world. (Wu et al., 2019) This is an emerging model for learning a broad range of complex tasks. Many computer games can be engaging and provide multi-sensory stimuli as the participant has to be involved in visual processing and incorporate the feedback during motor planning for the performance of goal-directed activities. (Sober \& Sabes, 2003)

These emerging rehabilitation technologies have the potential to improve clinical outcomes and enhance the active participation of children with neuro-motor deficits. This is because these platforms are engaging, motivating, and attractive to these young populations with neuro-motor discrepancies. There are varieties of therapeutic gaming and biofeedback platforms used to promote balance and locomotion rehabilitation in young children with CP. (Wu et al., 2019 and Dewar \& Johnston, 2014)

The use of force plates for training balance with visual biofeedback has been studied in the past. (Ledebt et al., 2005) This has shown a positive influence on balance control in children with CP. Developing this technology, the Biodex System is another assessment and treatment tool consisting of a standing platform embedded with forced plate sensors and a support handle 
for participants' safety. A computer display screen is used to provide feedback of the force plate signal especially the Centre of Mass (COM). The motion of COM is used to control the motion of the computer cursor during gameplay. This platform can be stationary or movable (which included random rotational perturbations in multiple directions) for testing and training of standing balance.

Benefits of the Biodex system for standing balance rehabilitation was reported in a study conducted by El-Shamy \& Abd El Kafy (2014) They recruited 30 children with mild (GMFCS Level $1 \& 2$ ) spastic diplegic $\mathrm{CP}$ in the age group of 10-12 years randomized to experimental or control groups. The control group received conventional PT including stretching, strengthening, standing, balance exercises, and gait training for 2 hours. The experimental group received a combination of conventional PT (90 minutes) and Biodex balance training (30 minutes). During training, children were instructed to move the computer cursor by shifting their COM to interact with the visual targets appearing on the screen (such as a flashing ball). This intervention was continued for 3 months at a frequency of 3 sessions per week. The experimental group showed a statistically significant improvement of $23 \%$ in the Pediatric Balance Scale (PBS) as opposed to $12 \%$ in the control group. The results of the Biodex fall risk assessment (while quiet standing for 20 seconds on the static surface with eyes open) demonstrated 3 times decreased fall risk in the experimental group than in the control group. Also, in the experimental group, there was a 5 times better improvement of standing balance assessed by the Biodex dynamic limit of stability testing module in comparison to the control group. (El-Shamy \& Abd El Kafy, 2014) Other studies also reported the benefits of this system in augmenting balance training in children with mild to moderate CP. (El-gohary et al., 2017 and Abd El Kafy \& El-Basatiny, 2014) 
Kozyavkin et al.,(2013) studied the use of dance mat stepping games to improve the balance of 5 participants aged 7 to 11 years diagnosed with moderate CP (GMFCS Level 2). There are eight games developed for this platform. For example, the child had to step on the region of the $3 \times 3$ square mat (represented as a flower on the screen) where the butterfly appeared. The frequency of target appearance could be varied to increase the speed of stepping. The therapy was performed as a home program daily for 30 minutes for 2 weeks. After 2 weeks of intervention implementation, there was a 2\% improvement in PBS scores and a 12\% decreased time taken to complete the TUG. In addition, the four step squared test (FSST) was decreased by $7 \%$ and the root mean square (RMS) on stabilometry was reduced by $12 \%$. The game events being similar in all games and requiring the participants to keep stepping would disinterest the participants quickly. Thus, there is a lack of engagement and motivation factors in this platform for using it clinically for long-term rehabilitation in CP. (Kozyavkin et al., 2013)

Hsieh, (2018) studied the effect of a PC gaming platform to address the rehabilitation of standing balance in children with CP. This PC gaming platform consisted of a joystick manipulandum with variable diameter handles secured on a tabletop. The motion of the handle controlled the motion of the computer cursor/game paddle to play a variety of PC games. The children were instructed to shift their weight while moving the handle of this manipulandum that had a variable load (resistance) ranging from 0.5 to $2.5 \mathrm{lbs}$. This proposed PC gaming platform provided trunk movements in 3 directions. They recruited 40 participants aged 5 to 10 years with GMFCS levels 2, 3, and 4 and randomized them to either control or experimental groups. Both groups played PC games while standing on a fixed surface and holding on to their respective controllers. The experimental group participants used the custom manipulandum and the control group participants used a standard optical mouse, which limits the movement to hand, 
and arm. Both groups continued PC gaming intervention at a frequency of 5 times a week for 12 weeks. The Centre of Foot Pressure (COP) Analysis in the experimental group during quiet standing on a fixed surface with eyes open demonstrated that there was a $4 \%$ decrease in AnteroPosterior (AP) sway, 3\% decrease in sway area, and 6\% decrease in sway velocity. Whereas only $1 \%$ decrease in AP sway, $1 \%$ decrease in sway area, and $<1 \%$ decrease in sway velocity in the control group were noted. Besides, the Berg Balance Scale (BBS) assessment showed significant improvement in the experimental group of $4 \%$ as opposed to $1 \%$ in the control group. The advanced balance skills assessed using the Fullertone Balance Scale showed no significant improvement in both groups. (Hsieh, 2018) These minimal changes might be attributed to lack of the additional balance costs during dependent balance training (as the children were holding on to the mouse/handle while being trained and no compliant surface training was added to therapy).

Sandlund et al., (2011) studied the effect of using a low-cost commercial interactive gaming platform as a home-based intervention to enhance the motivation level in children with $\mathrm{CP}$ and improve the level of physical activity in these children. They used Play Station 2 and Eye Toy Game 3 that comprised 20 games for interactive play at home. Fourteen children in the age group of 6 to 16 years (average 10 years and 11 months) diagnosed with mild to moderate $\mathrm{CP}$ (GMFCS Level 1, $2 \& 3$ ) were recruited for this study for the duration of 4 weeks. The SenseWear Armband was used to track the Total Energy Expenditure (TEE). The data on TEE demonstrated a statistically significant improvement in energy expenditure by a 5\% increase in calories burnt per day, steps taken increased by 13\%, and time over 3 MET - physical activity duration increased by $14 \%$. The review from parents was taken to estimate the qualitative results of this study. This intervention demonstrated a significant improvement in participant adherence 
to rehabilitative use of gaming and showed enhanced physical activity levels. (Sandlund et al., 2011)

Nintendo Wii Fit gaming system with balance board was a popular platform used for standing balance rehabilitation in children with CP. (Wu et al., 2019, Gatica-Rojas et al., 2016, Gatica-Rojas et al., 2017, Gatica-Rojas et al., 2017(2), AlSaif \& Alsenany, 2015, Sajan et al., 2017, Sharan et al., 2012, and Deutsch et al., 2008). Gatica-Rojas et al., (2017) examined the effect of exercise using the Wii Fit gaming system on standing balance performance of children with mild to moderate CP (GMFCS Levels $1 \& 2$ ). They recruited 32 children in the age group of 7 to 14 years and randomly assigned them to Standard Physical Therapy (SPT) and Wii-Fit therapy groups. Children in the SPT group received flexibility, strengthening, and balance exercises for 40 minutes each session. Wii-Fit therapy protocol consisted of 30 minutes of playing several Wii-Fit games, which required the children to perform right and left lateral weight-shifts. Both the groups received their respective sessions 3 times a week for 6 weeks. The magnitude of COP displacement in standing eyes-open was used as the primary outcome measure. Following the intervention, they observed a $26 \%$ decrease in COP displacement standing eyes open task in the Wii-Fit group whereas they observed a 9\% increased COP displacement in the control group. To note, there were no significant changes observed for the testing in the eyes-closed condition in both groups. (Gatica-Rojas et al., 2017)

Tarakci et al., (2016) also studied the effect of Nintendo Wii- Fit video gaming for the rehabilitation of balance in children with $\mathrm{CP}$ in the age group of 5-18 years. They recruited 15 children each in 2 groups. The control group received conventional PT for 50 minutes including strengthening, standing exercises on a balance board with different surfaces, weight shifts, minisquats, activities on the trampoline, and walking activities. The Wii Fit gaming group received a 
similar exercise protocol but the balance exercises were replaced by Wii Fit games for 20 minutes. These games focused on right and left lateral weight shifts. Both groups received their respective therapy twice a week for 12 weeks. The results demonstrated statistically significant improvement in Functional Reach Test, TUG, 10-meter walk test, and 10 stair climbing test in both groups. By considering the percentage of the change in both the groups, it was observed that the Wii-Fit group improved around twice better than the control group. (Taracki et al., 2016)

The Xbox Kinect gaming system is another commercial entertainment system that has gained interest from researchers and therapists for balance training for children (Jun et al., 2018, and Cheung et al., 2013) as well as the elderly. (Ayed et al., 2018 and Chanpimol et al., 2017). Luna-Oliva et al. (2013) performed a case series study using the Kinect gaming system for rehabilitation of standing balance. They recruited 11 children with mild to moderate $\mathrm{CP}$ (GMFCS levels 1 and 2) aged 4-11 years. The children played Xbox Kinect games along with their regular conventional physiotherapy. The children were instructed to use arm movements to catch balloons or to hit a tennis shot, or lower limb movements like jumping, stepping to stamp on objects or stepping in place to simulate walking in the virtual world, trunk movements like twisting and turning in place to avoid the targets shooting at them, etc. The children performed gaming for 30 minutes each session, twice a week for 8 weeks. The results showed a significant improvement of a 9\% increase in the Pediatric Reach Test distance, a significant improvement of $5 \%$ in the gross motor skills. The time taken to complete a 10 -meter walk test was also significantly reduced by $7 \%$ post-treatment using Kinect. (Luna-Oliva et al., 2013)

Camara Machado et al., (2017) conducted a study to investigate the effectiveness of exercise using the Kinect gaming system on balance in children with CP. They recruited 28 children in the age group of 3 to 12 years, diagnosed with mild to moderate CP (GMFCS levels 
1-3). The children were instructed to play several Xbox Kinect games, which involved movements of arms, trunk, and lower limbs. For instance, children were expected to move their arms and step sideways to catch the falling objects from the top or step in place to simulate walking through a virtual environment. The children played these games for 40 minutes, twice a week for 8 weeks. The GMFM scores demonstrated a statistically significant improvement of 5\% in the performance of gross motor skills. (Camara Machado et al., 2017) 


\section{Chapter 4: Statement of Research Problem/Gap:}

Various forms of commercially available digital media platforms include the Nintendo Wii, Microsoft Xbox Kinect, and Play Station. The Wii-based balance rehabilitation protocol improved the participant's level of participation but did not improve neuro-motor balance control function in children with CP. The XBOX Kinect-based avatar games showed mild to moderate improvements in gross motor function but no significant improvements were seen in balance function. The Play Station based protocol improved the children's physical activity level and their adherence to therapy but did not show a significant impact on the balance performance of children with CP. Therefore, most of these digital media-based protocols did not show any significant improvements in neuro-motor control for balance function in children with $\mathrm{CP}$ although the participation levels and therapy compliance were increased significantly due to the addition of game-play to the therapy protocols based on digital media platforms.

These gaming systems were designed for entertainment (Sandlund et al., 2011) and often require advanced balance skills to play games. (Hsieh, 2018, Taracki et al., 2016, and Camara Machado et al., 2017) This is appropriate for children with mild forms of CP (i.e. GMFCS level 1) and makes the use of this platform difficult for moderately or severely affected children. Moreover, the majority of studies evaluated the therapeutic benefits of interventions on children affected with mild forms of CP i.e. GMFCS level 1. It was observed that GMFCS level 1 comprised $45 \%$, GMFCS level 2 comprised $44 \%$ of the study population, GMFCS level 3 comprised 9\%, and GMFCS level 4 comprised only $3 \%$ of the study population. This makes it difficult to generalize the results of these studies to the population affected with moderate and severe forms of $\mathrm{CP}$. 
Most studies focusing on balance rehabilitation in this population, recruited children between $7-12$ years, and only less than $1 \%$ of the study population was aged $<6$ years. The majority of brain development occurs in early childhood. Thus, the rehabilitation initiated earlier than 6 years of age yields the best outcomes of therapeutic interventions. (Novak et al., 2017, Reid et al., 2015, and Grigoriu et al., 2021)

Most studies do not mention the exact amplitude and frequency of movements practiced during the therapy sessions. This makes it difficult to determine the balance cost and the therapeutic value of these interventions. When a child is performing game-assisted exercises, the amount of movement required to achieve game targets, the number of repetition of the tasks performed during game-play, the surface on which the standing balance tasks were performed, etc. has an important role to play in determining the balance cost of these exercises.

In summary, these technology-assisted platforms provide a fun, engaging, motivating medium for balance rehabilitation in children with $\mathrm{CP}$. These platforms have been proven to be effective in improving children's adherence to regular long-term physical therapy. (Bonnechère et al., 2017) Most of the study protocols used commercially available digital media platforms such as the Nintendo Wii and Xbox Kinect. These platforms provide a variety of gross motor exercises for upper extremity movements along with some truck control and balance control exercises. Unfortunately, these platforms are unable to detect, record, and assess the precise balance control movements and do not involve compliant surface balance training. 


\section{Chapter 5: Development of Computerized Game-aided Rehabilitation (CGR) Platform:}

Based on these gaps in the literature, a low-cost, interactive, computer game-aided rehabilitation platform (CGR) has been developed. This platform was initially designed to address the patient population with balance and gait impairments along with a cognitive decline for dual-task training. The platform is developed to be used as a therapeutic medium for indirect balance or gait training with simultaneous cognitive training. Dual-tasking (DT) is defined as the concurrent performance of motor-motor or motor-cognitive tasks that would have interference with each other. (Leland et al., 2017) For instance, reading while walking or simultaneously texting and walking or shopping down the aisle in a store are examples of motor-cognitive dual tasks. This platform uses the same concept of dual-task training. The patients have to control their balance performance while playing cognitively challenging games. Here, maintenance of balance is a sensory organization-motor task that is simultaneously performed with gaming, which is a cognitive task. The performance of these activities simultaneously (i.e. DT) would interfere with the performance of each of these tasks and thus make activities more challenging.

The CGR platform consists of a wireless, miniature, inertial motion mouse connected to a computer/laptop using a wireless USB (Therapy Mouse, Mobility Research, Az, USA). This mouse can be attached to any object or body part using simple Velcro straps to teather the cursor movement of the laptop/computer to the movement of the object or body part.

For this study, the mouse is attached to a cap/headband. The games are controlled by the use of hands-free, head rotation movements. The participants are engaged in commercially available, fun, interactive, cognitive computer games while the performance of challenging 
balance exercises in standing on a variety of compliant surfaces like a sponge, air bladder, wobble board, etc.

The CGR platform also includes a designed rehabilitation assessment game that is capable of monitoring a child's cognitive performance during gameplay. The electronic records from the assessment game quantify the visual-spatial skills and some executive-cognitive functions. When this information is coupled with the knowledge of their balance performance, an estimate of dual-tasking capabilities can be obtained.

The balance performance is evaluated by measuring the displacement of the center of foot pressure (COP) using a commercial pressure mapping system - flexible Force Sensor Array (FSA) Mat (Vista Medical Ltd., Winnipeg Manitoba) with a Sampling frequency of $86 \mathrm{~Hz}$. This FSA mat has 256 piezo-resistive sensors embedded in it. Each sensor can record an area of around $3 \mathrm{~cm}^{2}$. This pressure mat was used to evaluate standing balance for all participants over the ground and on the sponge surface. All participants stood on a 6-inch thick square sponge with a length of 20 inches and a density of approx. $25 \mathrm{~kg} / \mathrm{m}^{3}$ with a wooden plank placed on top of the sponge. (Szturm et al., 2014, Bhatt, 2018, Szturm et al., 2017, and Nankar et al., 2017) With this method balance performance can be determined while standing on a variety of fixed and compliant support surfaces like a sponge, balance disks, air bladders, etc. The change over time in both these outcomes provides timely feedback on the dual-tasking capabilities of the child.

The program is based on the theoretical grounds and current research documenting the benefits of computer-aided learning tools, exercise gaming applications in rehabilitation for influencing engagement and adherence. (Deutsch et al., 2017) The use of this game-aided platform coupled with traditional balance exercises on compliant surfaces may make the 
repetitive balance control exercises fun and engaging for children with CP. This platform uses the commercially available fun, interactive computer games and does not require a limited set of proprietary games of this platform to be designed, as does Kinect or Nintendo. Therefore, therapists and children get to choose from a large variety of existing common and modern computer games.

After a review of literature on the important principles of physiotherapy, the CGR platform was developed with 3 major principles embedded in this platform. The first principle is "engagement" - children are engaged in therapy by the addition of commercial games. The second principle incorporated is "repetition" - children are involved in repetitive task practice during game-play while standing on compliant surfaces by challenging their limit of stability (LOS) to achieve desired targets. Lastly, "target-specific training" - for balance and dual-tasking being another important principle that influenced the outcome of rehabilitation. (Sincero, 2011)

Encouraging results have been observed in community-dwelling older adults, (Szturm et al., 2011 and Betker et al., 2006) and patients with traumatic spinal cord and brain injuries (Betker et al., 2007) while implementing CGR protocol for balance rehabilitation. The successful trials of gait training with CGR in patient populations with neurological conditions like Parkinson's, Stroke, etc., and the hand-function improvement using CGR hand and arm rehabilitation program in children with CP and ABI (Kanitkar et al., 2017) are reported in the past. This has interested us to take this study further and investigate if the CGR platform has a beneficial effect for balance training in children with CP \& ABI. 


\section{Chapter 6: Purpose \& Objectives of the Study:}

\subsection{RESEARCH PURPOSE:}

The purpose of this pilot project is to generate evidence for the feasibility of conducting a full-scale Randomized Controlled Trial (RCT) for evaluation of the effectiveness of the CGR platform for balance rehabilitation in young children with $\mathrm{CP}$.

\subsection{RESEARCH OBJECTIVES:}

RESEARCH OBJECTIVE 1: Development of known group validity

Examination and comparison of the effect of Dual-Task Interference (DTI) on physical demands [standing balance on the fixed surface (floor) v/s standing balance on the compliant surface (sponge)] and on cognitive performance measures under single and dual tasking conditions in children with CP and TD children.

RESEARCH OBJECTIVE 2: Estimation of the feasibility of the use of the CGR platform in the rehabilitation of balance in children with $\mathrm{CP}$.

The recruitment rate, number of dropouts, number/percentage of sessions attended, number/percentage of sessions completed, any adverse events (pain, falls, etc.), number of protocol deviations \& the reasons for the same, etc.

Evaluation of participant experience and acceptability of the CGR platform for balance rehabilitation in children with $\mathrm{CP}$. The parents will be asked for consent before the intervention whether they would like to be interviewed at the end of the intervention to get an insight into 
their experience with the CGR protocol. They would be encouraged to share their views and comments for modifications in the protocol if any required for a better outcome. This would be done to obtain the evidence on acceptability, engagement, the motivation level of the child, and adherence to therapy. The feasibility of the assessment tool embedded in the gameaided rehabilitation platform and the usability and appeal of the protocol will be examined. Perception/acceptance of the parents and children measures the outcomes of the intervention provided. Intended and unintended outcomes as perceived by the parents will be observed and analyzed. The qualitative results of this study would help us reinforce and support the quantitative findings.

\subsection{RESEARCH HYPOTHESIS:}

For the first objective of this study, it is hypothesized that the CP group will show more DTI affection on balance and cognitive performance as compared to the typically developing children.

For the second objective of this study, it is hypothesized that it would be feasible to use CGR in Balance rehabilitation in young children with CP. It is also hypothesized that the CGR application for balance rehabilitation in young children with CP would be acceptable, accessible, engaging, and user-friendly. 


\section{Chapter 7: Methods and Protocols:}

For this study, we have recruited 50 participants - 25 children with CP and 25 typically developing children for the known group validity objective. In addition, 16 participants affected with CP divided into two groups of 8 children each were enrolled for balance training. One group received the experimental game-aided balance DT training program and the other group received the usual conventional balance-training program. Using a process of randomization, each child was assigned to the experimental $(\mathrm{XG})$ or control $(\mathrm{CBG})$ group after the initial assessment and screening.

The participants were randomly assigned to the two groups using the sealed envelope method of randomization. The use of this randomization method allowed us to maintain the Allocation concealment during the process of recruitment of study participants.

These randomly assigned participants in the active control arm $(n=8)$ received a typical conventional physical therapy program and the participants in the experimental arm $(n=8)$ received the computerized game-aided balance training (CGR) program.

Each group received their respective protocol for 12 weeks at a frequency of three therapy sessions per week. Before the treatment, sessions began, and after the end of 12 weeks, a blinded clinician conducted assessment sessions with the participant. In addition, we performed regular assessments to mark the progress to date and make necessary changes to the protocol as required subjectively for each participant in both groups. 


\subsection{Study Design:}

Mixed-Method Pilot Study Design.

\subsection{Prevention of Bias:}

The commonly encountered Risk of bias with this kind of study design includes selection and observation bias. (Mansournia et al., 2017) In this study, the use of the Sealed Envelope Method of randomization and allocation concealment while treatment group allocation prevented Selection Bias during the process of recruitment. In addition, the blinding of the assessor who performed the pre and post-12 weeks' assessment prevented the Observation Bias that could have occurred.

\subsection{Sample Size:}

50 participants in 2 groups - i.e. 25 per group i.e. typically developing (TD) children and children affected with $\mathrm{CP}$ for Objective 1 and 16 participants (children affected with $\mathrm{CP}$ ) $-\mathrm{n}=8$ in each group for other objectives of the study.

\subsection{Ethics Approval \& Informed Consent:}

Ethical clearance for the study has been obtained from the Health Research Ethics Board (HREB), University of Manitoba, Winnipeg, Canada, and the Institutional Ethical Committee of S. D. M. College of Medical Sciences and Hospital, Dharwad, India. Clinicians working in the Outpatient Department of Physiotherapy, Shree Dharmasthala Manjunatheshwar (S.D.M.) College of Medical Sciences and Hospital, Dharwad, Karnataka, India blindly recruited the participants. Parents of the participants were approached for consent to take part in this study. Further, when parents had any queries regarding the study, they were reported to the principal 
investigators. The parents were reassured that information will be kept confidential and will be used only for study purposes.

\subsection{Clinical Trial Registration:}

This study is registered at ClinicalTrial.gov with the Identifier \# NCT03873441.

\subsection{Participants:}

For the known-group validity objective of the study, 50 participants (25 typically developing children and 25 children affected with $\mathrm{CP}$ ) were recruited. These participants were asked for a commitment of 45-60 minutes for one assessment session.

For the second objective of the study, 16 children diagnosed with $\mathrm{CP}$ ( $\mathrm{n}=8$ for experimental and control groups) between the ages of 3-9 years old with the following inclusion and exclusion criteria were recruited for this study. The consent was obtained for a time commitment of 45-60 minutes per session thrice a week for 12 weeks.

The following Inclusion and exclusion criteria were considered during the process of recruitment:

For Typically Developing Children:

\section{Inclusion Criteria:}

(1) Typically developing children with no history of neuro-motor medical conditions,

(2) Age group - 3-9 years

\section{Exclusion criteria:}

(1) Recent fracture or injury affecting the functioning of the participant to perform the assessment tasks. 
For Children affected with CP:

\section{Inclusion Criteria:}

(1) Children with a confirmed medical diagnosis of Cerebral Palsy or Acquired Brain Injury $(\mathrm{ABI})$

(2) Age group - 3-9 years,

(3) Gross Motor Function Classification System level - 1-3, (Bodkin et al., 2003) \&

(4) Modified Ashworth scale level 0 to +1, (Mutlu et al., 2008)

\section{Exclusion criteria:}

(1) Visual or auditory impairment such that they cannot see and interact with the video games,

(2) Secondary orthopedic complications due to NDD,

(3) Recent Botulinum toxin therapy (less than 6 months),

(4) Recent Surgical intervention,

(5) Cognitive impairment,

(6) Seizures, or

(7) Complex communication disorders.

\subsection{Recruitment:}

The study participants were recruited from 2 locations:

1. Pediatric Physiotherapy Out Patient Department (OPD) of Shree Dharmasthala Manjunatheshwar College of Medical Sciences \& Hospital (SDMCMSH), Dharwad, Karnataka, India.

2. Usha's School for Exceptional Children, Hubli, Karnataka, India.

The typically developing children were also recruited from the same locations including the siblings or friends and relatives (that met the inclusion criteria) accompanying the participants to 
OPD for therapy or at school. Additionally, the typically developing children were recruited by contacting the staff and faculty at the OPD and School and their friends and relatives.

\subsection{Instrumentation:}

- Gross Motor Function Classification System (GMFCS)

- Modified Ashworth Scale of Spasticity (MAS)

- Gross Motor Function Measure - 88 (GMFM-88)

- Peabody Developmental Motor Scale - Second Edition (PDMS-2)

- Pediatric Balance Scale (PBS)

- Computer Game-Aided Rehabilitation (CGR) Platform

1. Miniature Motion Mouse (Therapy Mouse, Mobility Research, Az, USA). (Picture 1)

2. Sports Cap/Headband attached with a Velcro strap (for mounting the miniature motion mouse) (Picture 2)

3. Repetitive Task Practice (RTP) Assessment Gaming Software

4. Neuro-Function Evaluation Research (NFER) Game Data Assessment Software

- FSA Pressure Mat (Vista Medical Ltd., Winnipeg Manitoba). (Picture 3)

- Python 2.7.14. Version of the Software for FSA data Analysis

- Microsoft Office - Microsoft Excel for qualitative and quantitative data processing

- IBM Statistical Package of Social Sciences (SPSS) Statistics Version 27 for analysis of the study data

- Wooden plank

- Compliant Surfaces (Picture 4) including:

1. Sponge

2. Air Bladder/Balance Disk 
3. Bolster

4. Wedge

5. Wobble Board

6. Therapy Ball
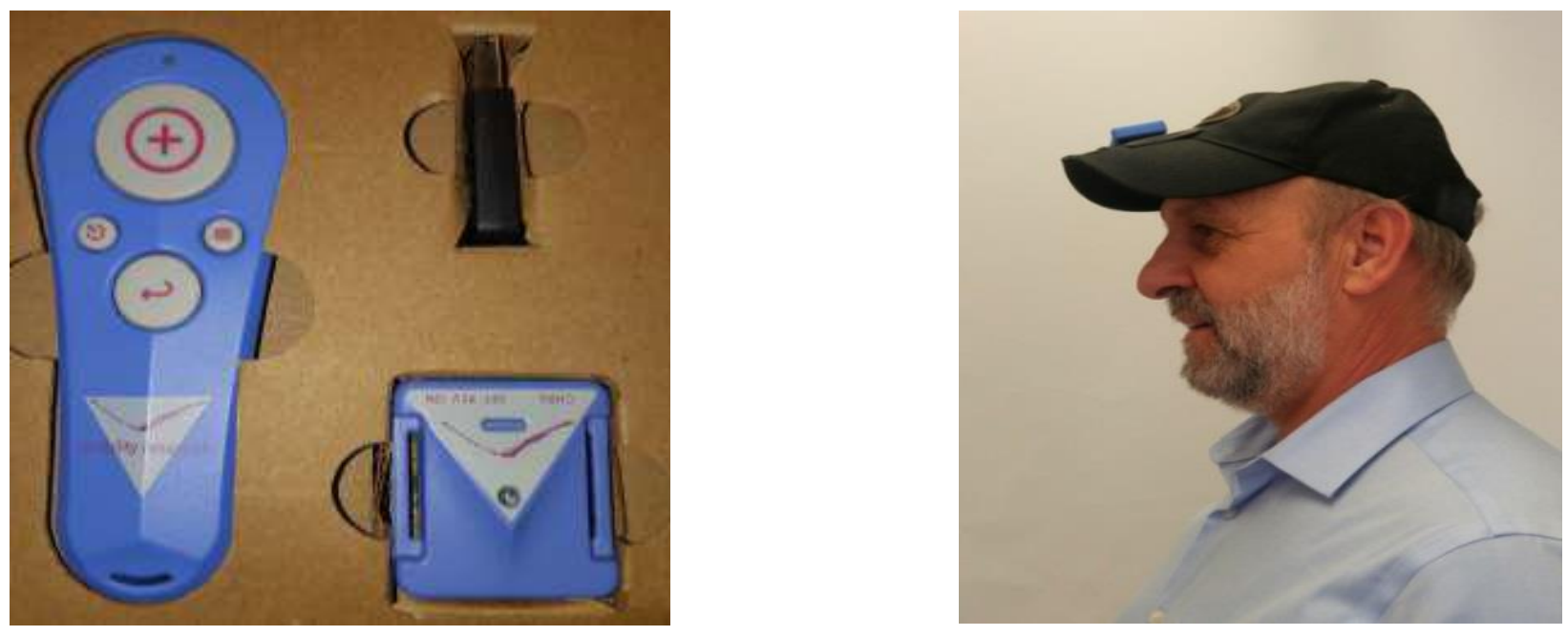

Picture 1 (Left) Miniature Motion Mouse (Therapy Mouse, Mobility Research, Az, USA).

Picture 2 (Right) Sports Cap mounted with Miniature Motion Mouse (attached with a Velcro strap) 

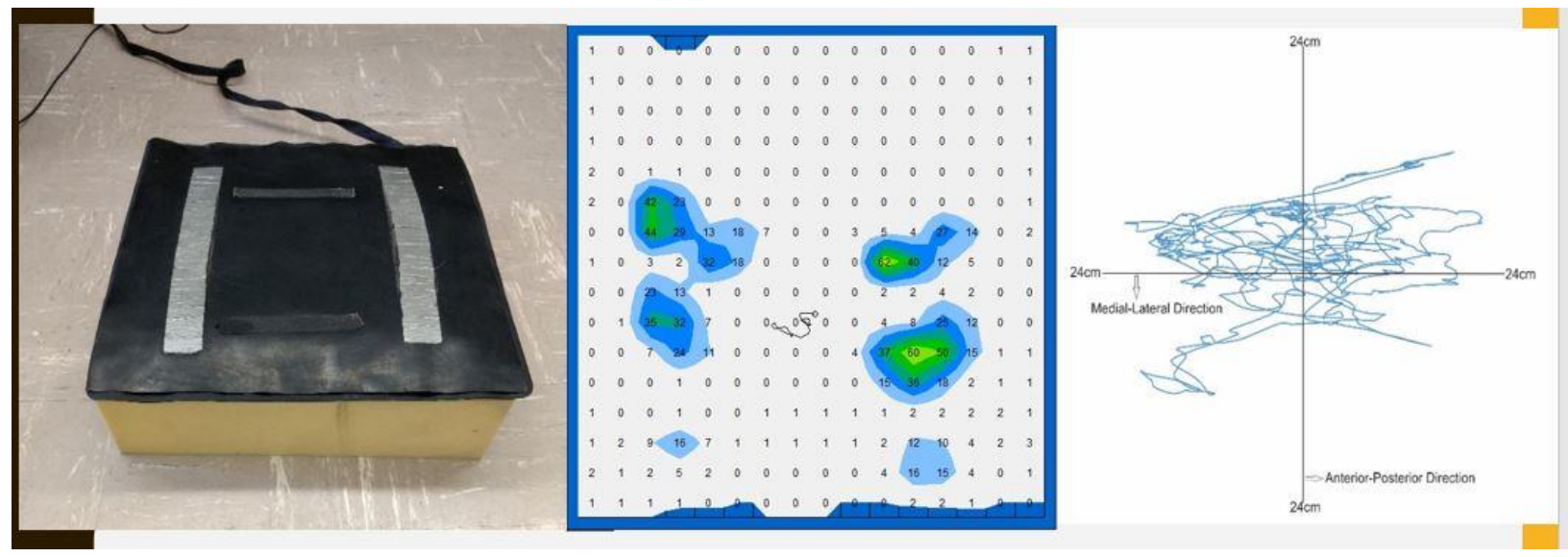

Picture 3 (From left to Right) FSA pressure Mat (Vista Medical Ltd., Winnipeg Manitoba) placed on a sponge surface, Snapshot of Pressure Mat Recording, and Center of Foot Pressure Excursion.

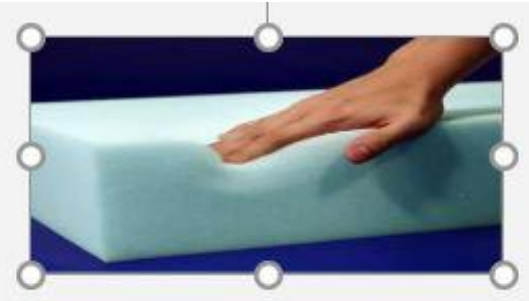

Medium Density Foam

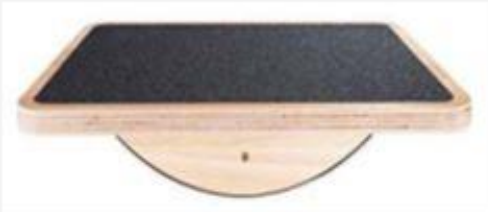

Wobble Board / Balance Board

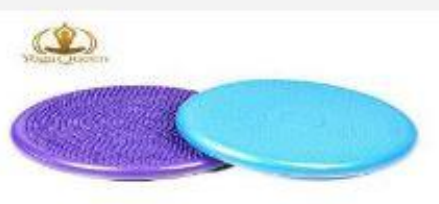

Air Bladder

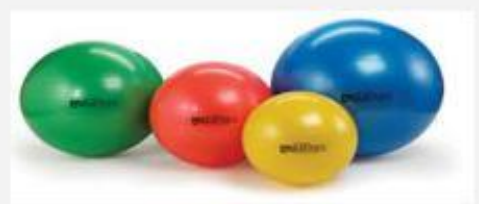

Vestibular Ball

Picture 4 Examples of Compliant Therapy Surfaces 


\subsection{Therapy Protocols:}

Two different blinded therapists delivered the therapy protocols to the experimental and control group participants. Each group received their respective protocol for a duration of 12 weeks at a frequency of three therapy sessions per week. Each session was 45-60 minutes long for both groups.

The starting point of the therapy (in terms of difficulty level) was determined after the baseline evaluation of the participant's standing balance capability. Conventional Physical Therapy Balance Training Protocol:

The control arm received the conventional physiotherapy protocol for balance training. This included:

(1) Active-assisted stretching exercises for postural control and improvement in dynamic stability,

(2) Balance and weight-bearing exercises with reach outs associated with trunk movements on a fixed surface gradually progressing to the unstable movable surface,

(3) Weight transfer exercises with reach outs, and

(4) Stepping exercises in different directions on a variety of surfaces.

\section{Experimental Computer Game-aided Balance Training Protocol:}

The experimental arm received the balance therapy protocol combined with computer gaming to encourage their active participation in the physical therapy program. The study participants in the experimental group were asked to stand on fixed and compliant surfaces and use the head rotation movements (wearing a headband/cap instrumented with the miniature motion mouse) to play various therapeutic yet entertaining games. This was done while rotating 
the head for controlling the game-play by tethering the cursor movements to the miniature motion mouse. The head movements used to control the cursor movements on the screen resulted in increased body sway and hence increased the balance cost of the exercises to result in an improvement in the balance control of participants. The speed, the amount, the number of head movements, and the amount of cognitive interference due to game events were graded by adjusting the difficulty level of game-play. The compliance of the surface on which the participant had to balance (i.e. balance cost) was varied depending on the child's standing balance ability and their limit of stability (LOS). For instance, the balance cost was varied depending on the child's initial assessment by using the progression of compliant surfaces from sponge to air bladder to bolster to wobble board to therapy balls.

Appropriate games that require tracking and various visuomotor cognitive processing or what is referred to as dual-tasking were used for therapeutic purposes. There are many common and modern computer games for this purpose to meet the skill levels and personal preferences of each child. For instance; 'brick buster games' like Action ball, Bricks of Egypt, etc., 'match 3 games' like Birds town, Digby's Donuts, etc. and shooting games like Brave piglet, Chicken invaders, etc. were used to vary the cognitive interference depending on children's preferences while dual-tasking during CGR balance therapy program.

The following is a summary of the experimental exercise protocol:

(1) Standing Balance exercises progressing from a fixed support surface to uneven and compliant support surfaces like a progression from standing over the ground to standing on a sponge and then on an air bladder as the child tolerates, i.e. increased balance cost.

(2) Progression then to balancing while performing the visuomotor and visuospatial game tasks of playing a variety of commercially available fun, interactive games using the head rotation 
movements (with mouse attached on headband/cap worn by the child) to control the games while balancing on a variety of compliant surfaces.

(3) Progression to single-leg stance and stepping activities as tolerated by the child while on compliant surfaces simultaneously playing games.

Challenging balance and cognition at all times during therapy is the key to improvement.

Progressions were made by modifying 3 aspects of the treatment:

1. By changing the mouse placements - like placing it on an object for increasing the moment arm or by placing it on a body part and using the body movement as the game controller. (Picture 5 \& 6)

2. By increasing the balance cost - like the use of different compliant surfaces with more degrees of freedom. For instance, a wobble board, a bolster, a balance disk, or a therapy ball.

3. By increasing the difficulty level of the game - which involved more optokinetic stimulus or faster and large amplitude movements, etc. (Picture 7)
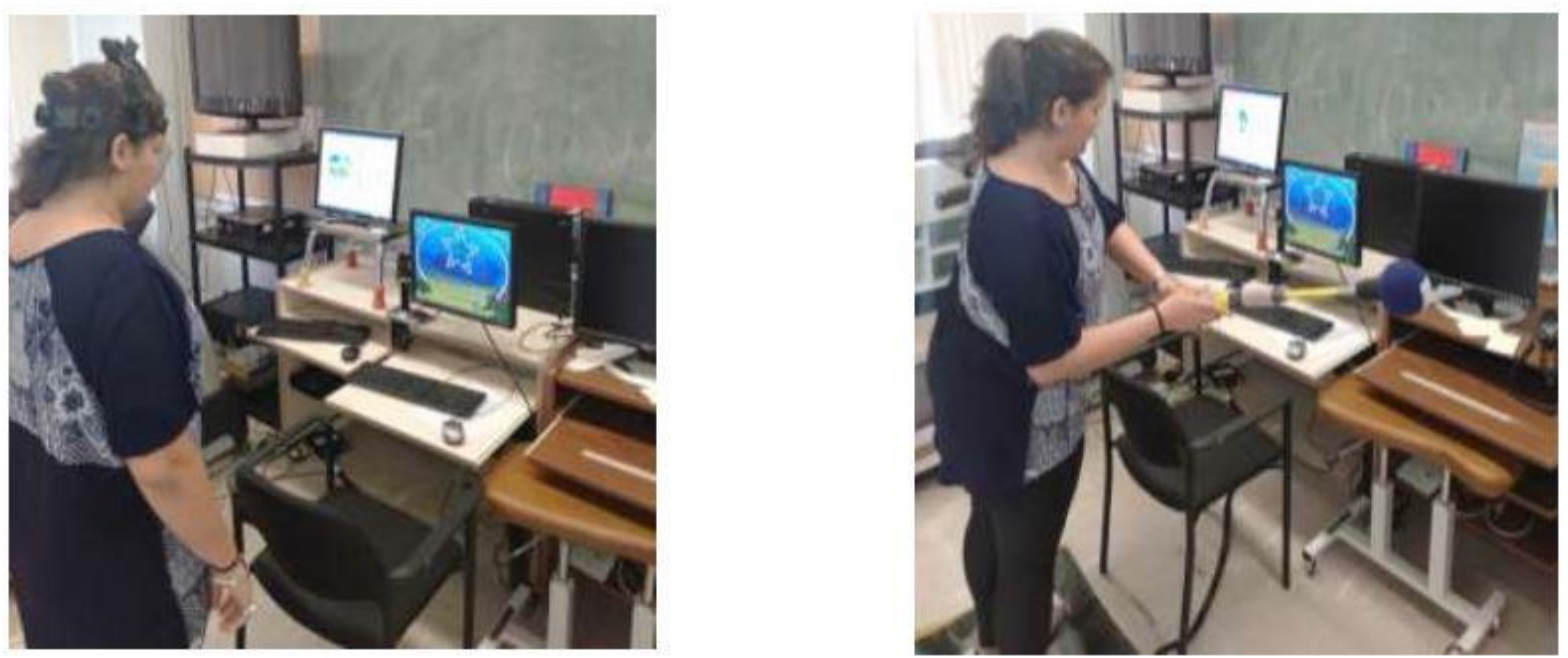

Picture 5 (Left) Dual-tasking with Miniature Motion Mouse Placement on head band. 
Picture 6 (Right) Progression of dual-tasking by use of bimanual handling for game control with mouse placement on object held in hands

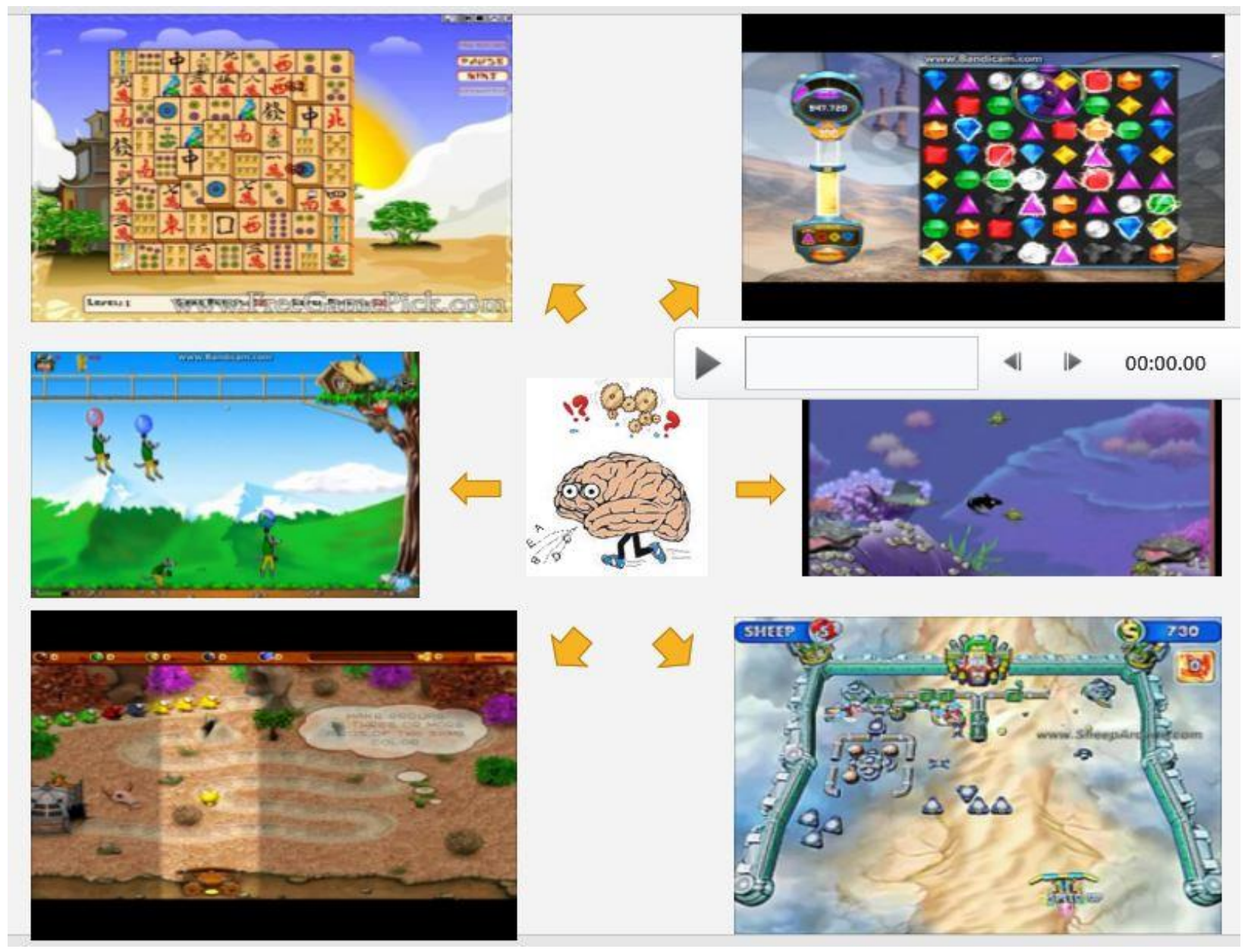

Picture 7 Snapshots of Variety of Therapeutic Games used for Dual-task Training.

\subsection{Primary Outcome measures:}

The primary outcome data for this study were collected by a blinded assessor at the start and after 12 weeks of intervention. The primary outcome measures used for this study were as follows: 
1. Peabody Developmental Motor Scale-Second Edition (PDMS-2): The PDMS-2 is a motor development assessment tool for children aged birth to 5 years of age. We have used the following sub-tests, Stationary and Locomotion. Example tasks include walking 5 meters forward and backward, walking up/down up a flight of four steps, etc. (Wang et al., 2006, van Hartingsveldt et al., 2005, and Zanella et al., 2021)

2. Gross Motor Function Measure-88 (GMFM-88): The GMFM-88 is an observational clinical tool designed to evaluate a change in gross motor function in children with $\mathrm{CP}$ aged between 5 months to 16 years. We have used standing and walking, running, and jumping components of the scale as our primary outcome measures of the study. Example tasks include catching and throwing a small sports ball, kicking a soccer ball in a particular direction. Standing on toes and one leg, walking a straight line for 10 steps. (Alotaibi et al., 2014 and Wei et al., 2006)

3. Pediatric Balance Scale (PBS): The PBS is a modified version of the Berg Balance Scale (BBS) for application of the balance assessment tool in children. It consists of 14 items that examine the functional balance required in ADLs in the pediatric population. Examples of the tasks include standing unsupported, standing with narrow base of support (BOS), tandem standing, turning, forward reach, etc. (Chen et al., 2013)

4. Test of dynamic balance performance: This is based on analysis of the center of foot pressure. The displacement of the Centre of foot pressure (COP) was recorded using a flexible Force Sensor Array (FSA) Mat (Vista Medical, Sampling frequency 86 Hz, 1100 sample frames collected in 30 seconds). Total Sway Path Length (TPL) and the Root Mean Square (RMS) of COP displacement in both the medial-lateral (ML) and anterior-posterior (AP) were computed during the 5 tasks (task duration set at 20 seconds each). FSA pressure mat was placed over the ground or on the sponge to evaluate the COP displacement during all the assessment 
tasks in standing. The tasks included four conditions of the modified Clinical Test of Sensory

Integration in Balance (mCTSIB) and dual-tasking (standing and performing various visuomotor tracking and cognitive games tasks) on a normal fixed surface and then on a compliant sponge surface (i.e. during increasing balance demands)

A total path length was computed for all tasks on both surfaces using this data. Root mean square (RMS) amplitude values of total COP displacement in both AP and ML directions were determined. A lower RMS amplitude value indicated reduced body sway and better standing balance. (Szturm et al., 2014)

\subsection{Assessment Protocol:}

The participant's balance was assessed during the performance of five tasks developed from mCTSIB on Fixed and Compliant surfaces each using the Pressure mapping system. The five tasks were:

1. Standing with Eyes Open

2. Standing with Eyes Closed

3. Standing \& Playing Visuo-Motor Tracking Game (VMT)

4. Standing \& Playing Easy Cognitive Game (CG1)

5. Standing \& Playing Difficult Cognitive Game (CG2)

4. Visuomotor tracking performance and Visuomotor cognitive game performance:

This was tested under different balance conditions (balance demands). A computer application with two assessment modules developed to guide two standardized computer tasks, i.e. visuomotor tracking (VM) and visuomotor cognitive game (CG) task was used for cognitive 
assessment of the participants. An inexpensive, commercial inertial-based motion mouse (Therapy Mouse, Mobility Research, Az, USA) secured to a sports cap or small plastic headband was to be worn by the children. The inertial mouse functions exactly like a standard optical computer mouse. With this simple method hands-free head pointing movements (i.e. head rotation) were used to control the motion of the on-screen computer cursor/game paddle. (Szturm et al., 2014, Bhatt, 2018, Szturm et al., 2017, and Nankar et al., 2017) Visuo-Motor Tracking (VM) task:

The goal here was to overlap the two moving objects on the computer display: as they move horizontally. The computer controlled the circle, which is the Target Object. It was configured to move horizontally (left to right) on the computer display for several cycles at a constant speed and amplitude. And, the participant controlled the rectangle, via the inertial motion mouse using head rotation movements. Continuous foveation was required to determine the separation and alignment of the two moving targets. This information (visual feedback) was used to produce corrective movements and maintain overlap between the two moving objects.

One trace in BLACK represented the computer-generated "Target Object" motion (ideal sinusoidal trajectory). The second trace in RED represented the participant's actual movements using head rotation. We observed each participant's individual tracking performance to analyze his or her engagement in the VM tracking task. All participants found to be involved for more than $25 \%$ of the computer-generated movement, were considered as engaged whereas all the participants failing to meet the $25 \%$ involvement were held as disengaged in the VM tracking task.

The Outcome measures used from the VM Tracking tasks are :

1. Average Residual Error (ARE) \& 
2. Engagement Frequency

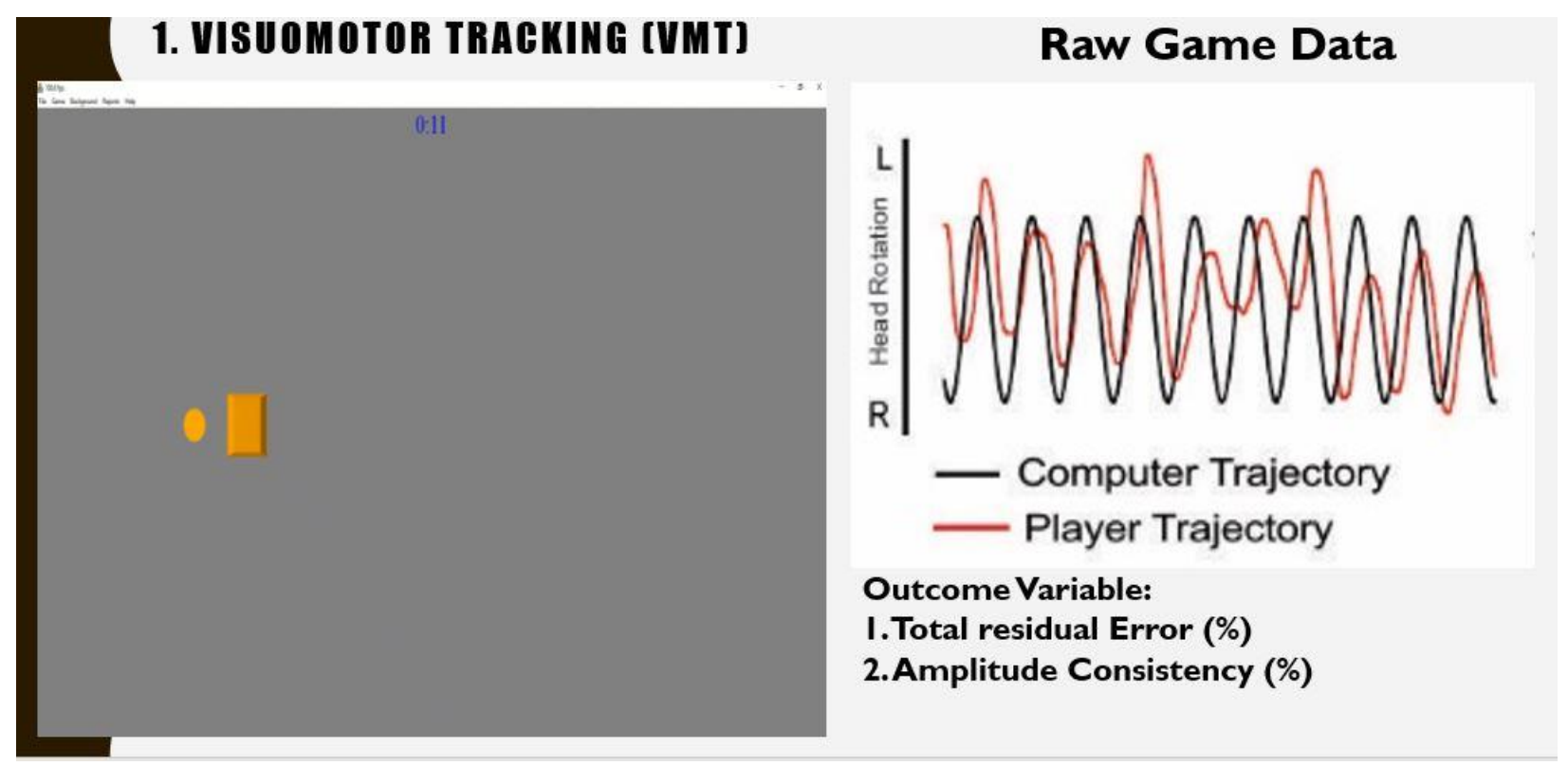

Picture 8 (Left) Snapshot of VM Tracking.

Picture 9 (Right) Visual of VM tracking Raw Data.

Visuo-Motor Cognitive Game (CG) Task:

The objective of this game was to catch the target object, which was the soccer ball, on the orange paddle, and avoid the distractor object, which was the dotted sphere. The orange paddle was enslaved to the head rotation movements of the participants by virtue of our wireless motion mouse. Every $2 \mathrm{sec}$ a target and a distractor fell from the top of the computer screen in a random direction. 60 seconds of game-play resulted in 30 movement responses.

CG was played in two different modes; the CG1 - involved no optokinetic background, and objects dropped with a straight trajectory whereas the CG2- involved a diagonal trajectory of objects falling from the top of the screen. 
The outcomes used from the cognitive target games were:

1. Success rate, which was the percentage of the total number of Target Objects caught in one game session.

2. Movement Error, which was the percentage of events when the game movements were in opposite direction than the actual target direction.

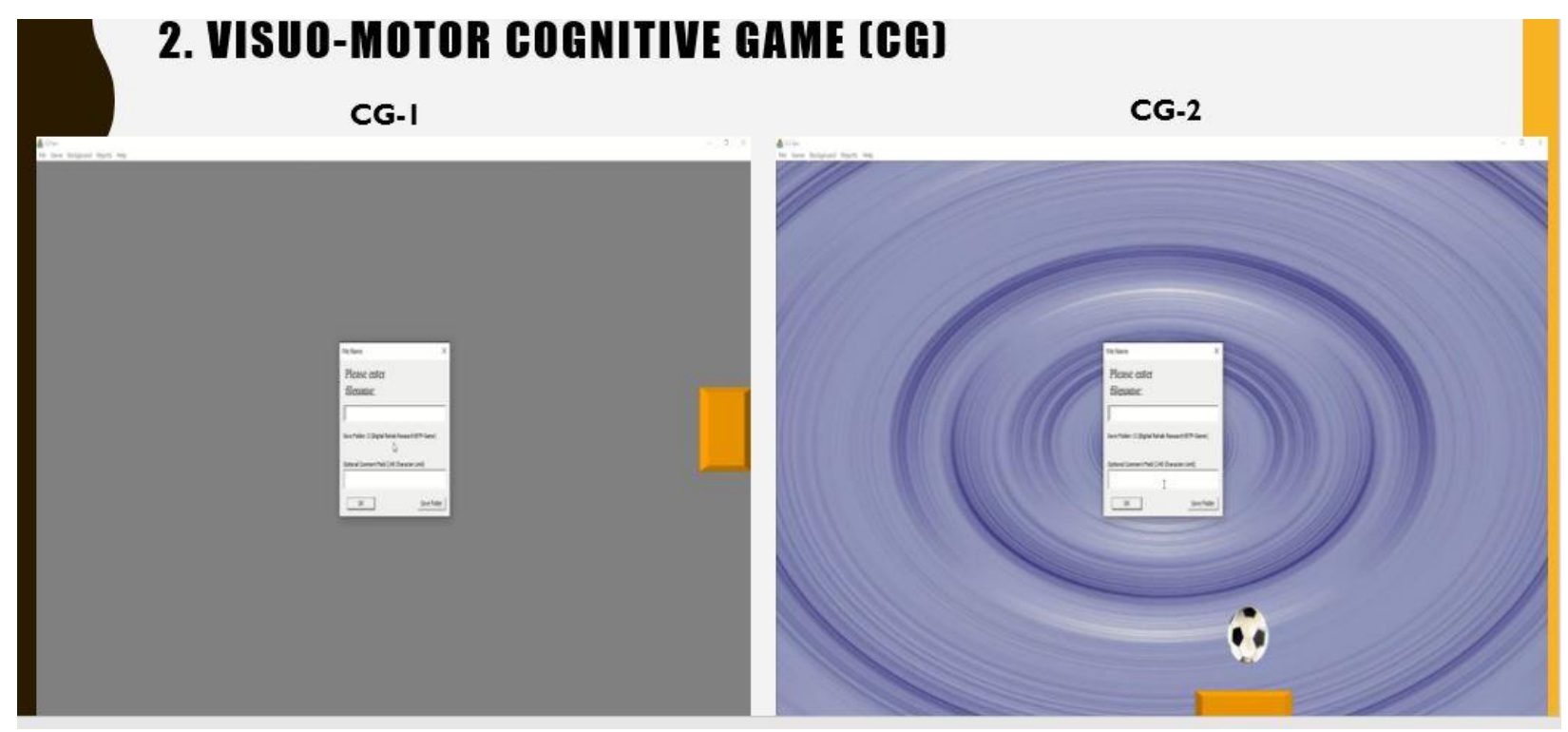

Picture 10 (Left) Snapshot of Easy Cognitive Game.

Picture 11 (Right) Snapshot of Difficult Cognitive Game 

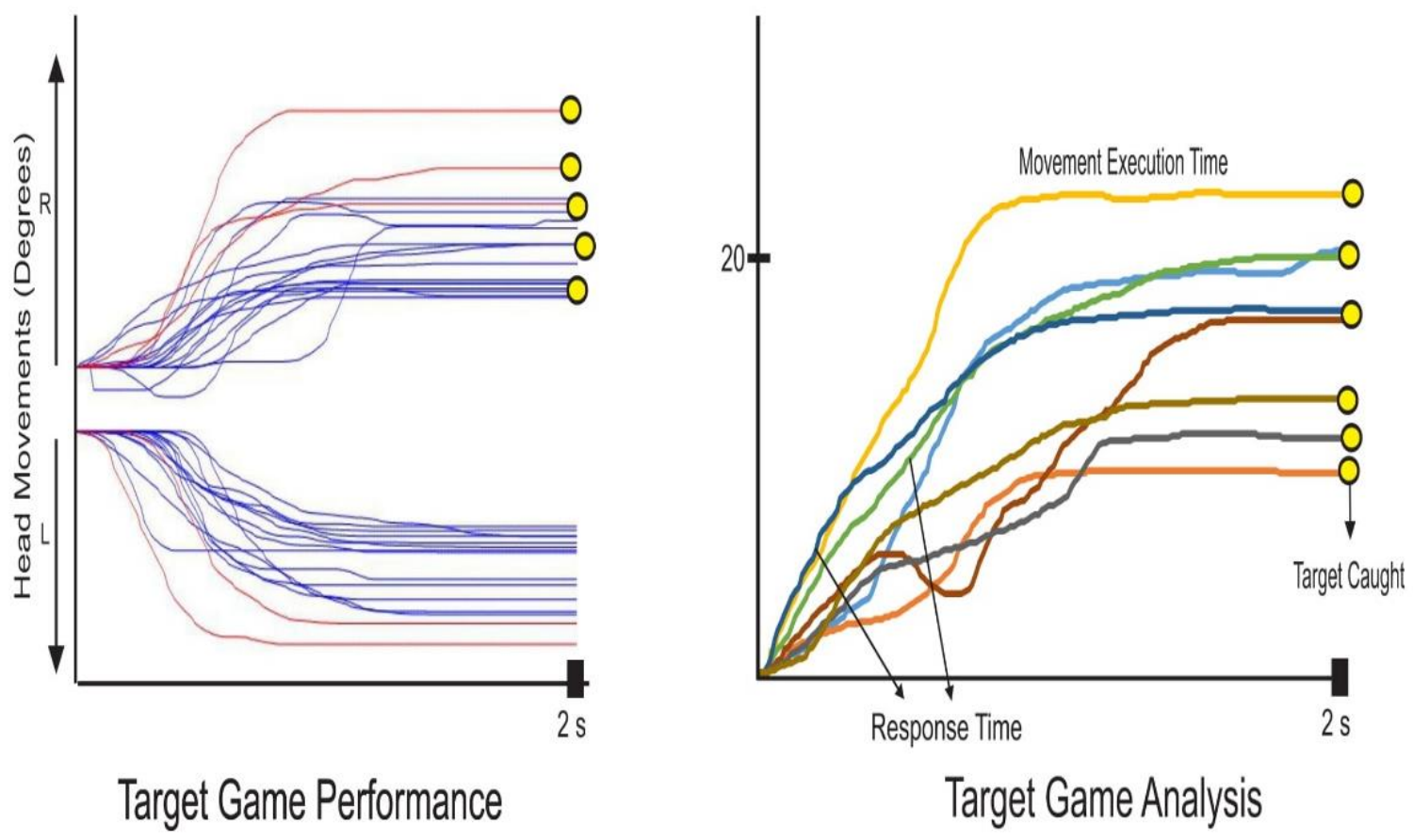

Picture 12 Visual of Cognitive Target Game Raw Data.

The analysis of COP displacement using a Pressure mapping system helped us compute the Total Path Length to evaluate the balance performance of the participants. The outcomes from the RTP game helped us assess the cognitive functioning of these participants. The combined analysis of the balance and cognitive performance data helped us estimate the dual-tasking capabilities of these participants. 


\subsection{Statistical Data Analysis:}

\subsection{2a Quantitative analysis}

For the first research objective of this study, we have examined the DTI effect on the physical performance (COP Displacement during standing balance tasks) and on cognitive performance (Visuomotor Tracking and Cognitive Target game performance) using unpaired ttests. We have used the software IBM Statistical Package of Social Sciences (SPSS) Statistics Version 27 for analysis of the study data. The independent variables here are the use of fixed and compliant surfaces \& the presence of pathology i.e. CP or no pathology i.e. typically developing children. The dependent variables here are the balance performance (i.e. the TPL) and the cognitive performance (i.e. game outcomes including Average Residual error (ARE), SR, and ME)

For the first half of second research objective of this study, we have used the observation analysis of the recruitment rate, compliance, reported adverse events, and the protocol deviations along with the reasons for the same for assessing the feasibility of application CGR for balance training in young children with $\mathrm{CP}$. We have also examined the effect of the intervention in both groups. The independent variables here are the time (pre vs post) and group (experimental vs control). The dependent variables here being the primary outcome scores from PDMS-2, GMFM-88, and PBS and the balance performance assessment measures from COP excursion data (i.e. the TPL) analysis, frequency analysis of the engagement of participants during VM tracking task, and the cognitive performance assessment measure from the CGR assessment gaming platform (i.e. game outcomes including SR and ME). 


\subsection{2b Qualitative Analysis:}

For the final part of the second research objective of this study, we have used an interpretive descriptive analysis using a general inductive approach. (Thomas, 2006) The parents of the participants in the experimental groups were interviewed using a semi-structured interview guide.

It is important to add qualitative methods to provide additional insight into how and why participants liked or did not like the CGR platform. (Tickle-Degnen, 2013, Hadi \& Closs, 2016, Cope, 2015, Almalki, 2016, Tsianakas et al., 2017, Driscoll et al., 2007, and Malina et al., 2011) The aim of the CGR platform and other game-assisted rehabilitation approaches is not only to provide information but also rather to stimulate participants in their process of practice. Therefore, information is also needed regarding how the use of technology can explain the engagement and involvement of the individual user. As per the literature review, having a very large sample size will result in the Saturation of the data. (Varpio et al., 2017) This is not the case in this study as the sample size here is eight participant's parents for the XG. On the other hand, triangulation is a result of having only one source or form of data i.e. homogenous data that is being taken care of by the addition of a second reviewer to review the data after evaluation by the interviewer ( $1^{\text {st }}$ reviewer). (Varpio et al., 2017 and Oleinik, 2011)

A blinded research therapist recorded parent's experiences and views at the end of the treatment period using a semi-structured interview guide (Consent was obtained at the beginning of the study). The interviews were conducted in the local language (Kannada) or the parents' preferred language. The interviews were conducted in a consultation room and were audiorecorded. In some cases due to inconvenience, the interviews were conducted over the telephone and the telephonic conversations were recorded. These interviews then were transcribed and 
translated to English for further Analysis using the Interpretive Descriptive Analysis method of Qualitative data analysis. The data was interpreted and organized into various themes and categories by the interviewer. The second reviewer then reviewed this for the development of unique codes or paraphrases that were not interpreted by the first reviewer. After this, both the reviewer discussed and sorted out the disagreements and came to mutual agreements on the unique codes and phrases. The data was then translated back to the same language in which the interview was conducted. This translated data was sent back to the parents for their review to prevent any misinterpretation of their views and experiences regarding the intervention. This member check was done to prevent any misrepresentation of the data from the interview and maintain its credibility. (Varpio et al., 2017 and Oleinik, 2011) 


\section{Interview Guide}

\section{Semi-structured Interview Questions for Parents of participants receiving computer games-aided balance training protocol:}

This guide focused on generating information on views and experiences of the parents about the benefits, expectations, likes and dislikes, and outcomes of using CGR for rehabilitation of balance in young children with $\mathrm{CP}$.

The following open-ended questions were asked of the children's parents:

(1) When you agreed to participate, how did you hope your child would benefit from the therapy program?

(2) Were there things about the game (or exercise therapy program) for your child you liked and things you did not like?

(3) What did you think about the computer games your child was asked to play? Did your child enjoy the games? Were there games that your child did not seem to enjoy?

(4) Did you feel that this therapy program helped your child?

(5) If you were provided with the right setting, would you want your child to continue with these exercises? 


\section{Chapter 8: Results:}

\subsection{Known-Group Validity Results:}

Table 1 presents the demographic details of all the participants $(n=50)$ for the known group validity part of this study. Each group had 25 participants (Typically Developing (TD) n=25 and Cerebral Palsy (CP) n=25) who participated in one assessment session for the known group validity objective of this study. Both groups did not differ in demographics at baseline as the mean age of participants in the TD group was 7.3 years; the CP group was 6.6 years, with no statistically significant difference (p-value - 0.70). The typically developing children were considered to be the GMFCS Level 1 for this statistical analysis and the participants in the CP group constituted a mix of GMFCS Levels 1 to 3 (GMFCS Level 1 - 10, GMFCS Levels 2 - 11, \& GMFCS Level 3 -4). The groups did not have any significant difference in the genders of the participants (p-value - 0.78).

Table 1: Demographic Table for the Known Group Validity Study:

\begin{tabular}{|c|c|c|c|c|}
\hline $\begin{array}{c}\text { Demographic } \\
\text { Detail }\end{array}$ & $\begin{array}{c}\text { TD Group } \\
\text { Mean (SEM) }\end{array}$ & $\begin{array}{c}\text { CP Group } \\
\text { Mean (SEM) }\end{array}$ & T-statistics & $\begin{array}{c}\text { Significance (2- } \\
\text { tailed) }\end{array}$ \\
\hline Age & $7.3(0.5)$ & $6.6(0.6)$ & 0.96 & 0.70 \\
\hline $\begin{array}{c}\text { GMFCS Levels } \\
\text { of participants }\end{array}$ & Level 1=25 & $\begin{array}{c}\text { Level 1=10, } \\
\text { Level 2=11, } \\
\text { Level 3=4 }\end{array}$ & -5.25 & 0.00 \\
\hline Gender & $11(\mathrm{~F}), 14(\mathrm{M})$ & $10(\mathrm{~F}), 15(\mathrm{M})$ & -0.28 & 0.78 \\
\hline
\end{tabular}


Table 2 presents the results of the unpaired t-test for the pressure mapping data (TPL) for all tasks on fixed and compliant surfaces. No statistically significant results were observed for the TPL data on the fixed surface. The effect size of most tasks was medium except for one task. The effect size was observed to be small for the CG1 task on the fixed surface. However, an increasing trend was seen for the tasks on the fixed surface, with increasing levels of difficulties.

The effect size was observed to be medium for EO, CG1, and CG2 tasks on the compliant surface with statistically insignificant differences between the groups. The effect size was observed to be large for EC and VM tracking tasks on the compliant surface with statistically significant differences between the groups. Here CP group demonstrated lower COP displacement (TPL) as compared to the TD group. Another observation was that the TPL mean for both the groups kept on increasing as the difficulty levels were increased for each task starting from a single task to the performance of dual-task with an increasing level of difficulty.

Table 2: Results of Unpaired T-test for Total Path Length (TPL):

\begin{tabular}{|c|c|c|c|c|c|}
\hline Task & $\begin{array}{c}\text { TD Group } \\
\text { Mean (SEM) }\end{array}$ & $\begin{array}{c}\text { CP Group } \\
\text { Mean (SEM) }\end{array}$ & T-statistics & $\begin{array}{c}\text { Significance } \\
\text { (2-tailed) }\end{array}$ & $\begin{array}{c}\text { Effect Size } \\
\text { (Cohen's d) }\end{array}$ \\
\hline EO_F & $47.7(6.1)$ & $54.5(7.1)$ & -0.73 & 0.47 & Medium (-0.21) \\
\hline EC_F & $48.7(5.6)$ & $65.6(12.5)$ & -1.23 & 0.22 & Medium (-0.35) \\
\hline VM_F & $52.9(6.0)$ & $61.6(8.3)$ & -0.85 & 0.40 & Medium (-0.24) \\
\hline CG1_F & $86.0(9.2)$ & $92.5(12.2)$ & -0.43 & 0.67 & Small (-0.12) \\
\hline CG2_F & $85.0(10.0)$ & $106.3(16.7)$ & -1.10 & 0.28 & Medium (-0.31) \\
\hline EO_C & $42.9(2.5)$ & $42.4(3.5)$ & 0.10 & 0.92 & Medium (0.03) \\
\hline EC_C & $50.4(3.1)$ & $41.7(2.9)$ & 2.03 & $\mathbf{0 . 0 5}$ & Large $(0.58)$ \\
\hline VM_C & $52.4(3.3)$ & $42.5(3.0)$ & 2.20 & $\mathbf{0 . 0 3}$ & Large $(0.62)$ \\
\hline CG1_C & $78.7(4.9)$ & $72.2(6.1)$ & 0.84 & 0.41 & Medium $(0.24)$ \\
\hline CG2_C & $76.2(4.8)$ & $68.3(6.5)$ & 0.98 & 0.33 & Medium $(0.28)$ \\
\hline
\end{tabular}


Figures $1 \& 2$, demonstrate the COP displacement (TPL) for both groups on both surfaces. Figure 1 demonstrates the performance of displacement of COP (TPL) on the compliant surface for both the groups where for EC and VM tasks, the CP group demonstrated less COP displacement (TPL) as compared to the TD group. Figure 2 demonstrated a relationship between the two groups for both the group performance of COP displacement (TPL) for all 5 tasks on the fixed surface.

\section{Figure 1: Bar chart for the Total path length for all tasks performed on Sponge Surface.}

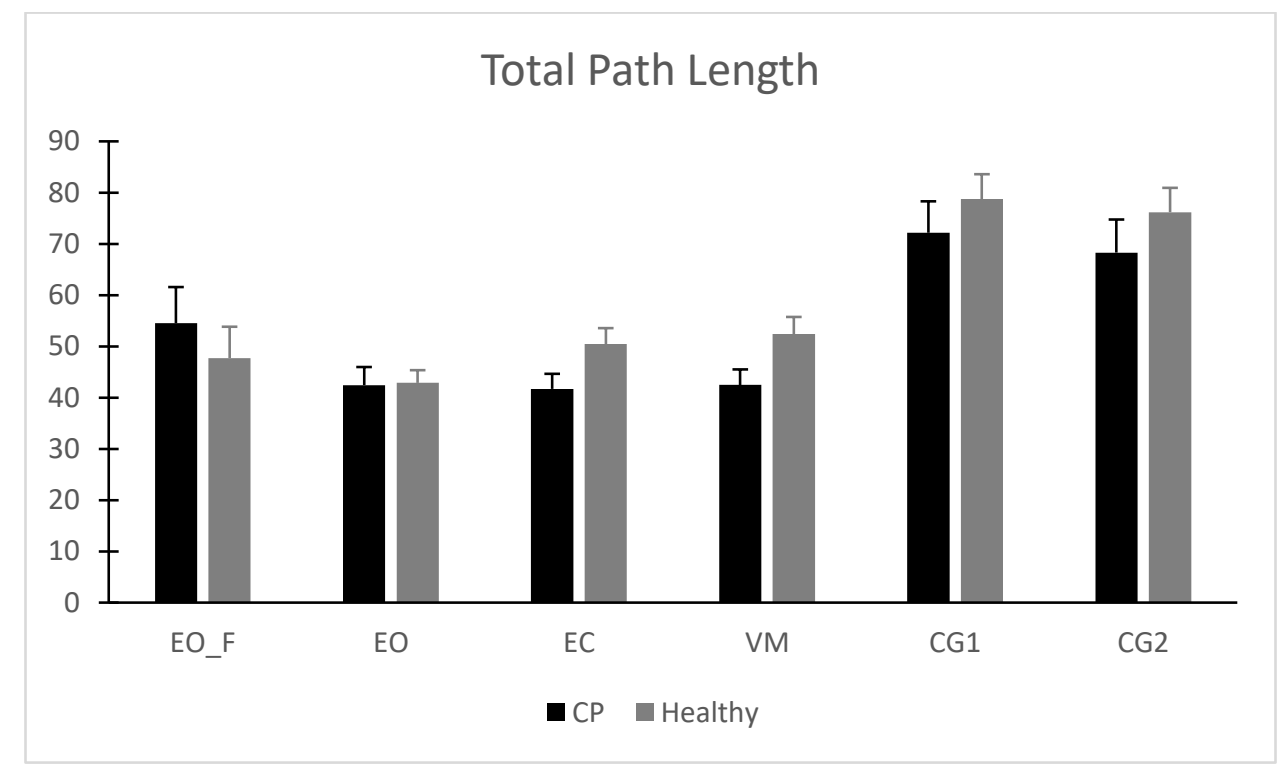

Figure 1 demonstrates the TPL for all tasks performed on the compliant surface for both groups where Series 1 is TPL for CP (Black Color) and Series 2 for TD (Grey Color). The Y-axis consists the TPL values and the $X$-axis demonstrates the 6 tasks i.e. EO_F - Eyes Open on Fixed Surface (for reference), EO - Eyes Open, EC - Eyes Closed, VM - Visuo-Motor Tracking, CG1 - Easy Cognitive Game, CG2 - Difficult Cognitive game. 
Figure 2: Bar chart for the Total path length for all tasks performed on Fixed Surface.

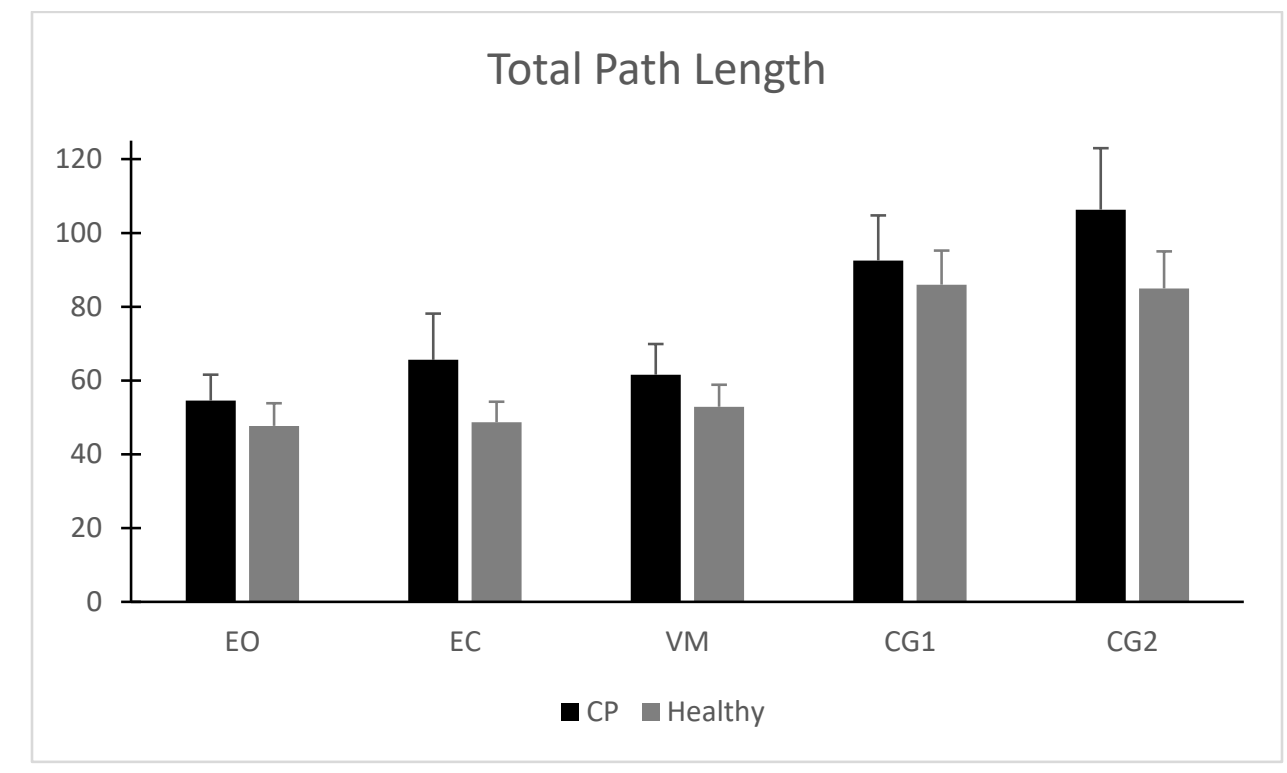

Figure 2 demonstrates the TPL for all tasks performed on the fixed surface for both the groups where Series 1 is TPL for CP (Black Color) and Series 2 for TD (Grey Color). The Y-axis consists the TPL values and the X-axis demonstrates the 5 tasks i.e. EO - Eyes Open, EC - Eyes Closed, VM - Visuo-Motor Tracking, CG1 - Easy Cognitive Game, CG2 - Difficult Cognitive game. 
Table 3 presents the unpaired t-test results for the peak-to-peak (P2P) displacement of the COP in the Medio-lateral (ML) direction. The results provide us an idea that the TD group demonstrated less excursion of COP in ML direction on the fixed surface as compared to the $\mathrm{CP}$ group. The EC, VM, and CG2 tasks results demonstrated statistically significant outcomes with large effect sizes. The EO task result demonstrated a large effect size but statistically insignificant results. The CG1 task result demonstrated a medium effect size with statistically insignificant results as well.

The results for the tasks on the compliant surface were all statistically insignificant with effect sizes being small for all tasks on the compliant surface except the EO task result that demonstrated a medium effect size on the compliant surface.

Table 3: Results of Unpaired T-test Results for Peak to Peak COP Excursion in $\underline{\text { Medio-lateral direction (P2P_ML): }}$

\begin{tabular}{|c|c|c|c|c|c|}
\hline Task & $\begin{array}{c}\text { TD Group } \\
\text { Mean (SEM) }\end{array}$ & $\begin{array}{c}\text { CP Group } \\
\text { Mean } \\
\text { (SEM) }\end{array}$ & T-statistics & $\begin{array}{c}\text { Significance } \\
\text { (2-tailed) }\end{array}$ & $\begin{array}{c}\text { Effect Size } \\
\text { (Cohen's d) }\end{array}$ \\
\hline EO_F & $9.1(0.3)$ & $9.9(0.3)$ & -1.84 & 0.07 & Large (-0.52) \\
\hline EC_F & $9.1(0.3)$ & $10.3(0.4)$ & -2.79 & $\mathbf{0 . 0 1}$ & Large (-0.79) \\
\hline VM_F & $9.2(0.3)$ & $10.1(0.3)$ & -2.07 & $\mathbf{0 . 0 4}$ & Large (-0.59) \\
\hline CG1_F & $9.6(0.3)$ & $10.1(0.3)$ & -1.09 & 0.28 & Medium (-0.31) \\
\hline CG2_F & $9.1(0.3)$ & $10.2(0.4)$ & -2.20 & $\mathbf{0 . 0 3}$ & Large (-0.62) \\
\hline EO_C & $9.6(0.2)$ & $10.0(0.3)$ & -0.93 & 0.36 & Medium (-0.26) \\
\hline EC_C & $10.0(0.3)$ & $10.1(0.4)$ & -0.37 & 0.71 & Small $(-0.10)$ \\
\hline VM_C & $10.1(0.2)$ & $10.0(0.3)$ & 0.25 & 0.80 & Small $(0.07)$ \\
\hline CG1_C & $10.2(0.2)$ & $10.2(0.3)$ & -0.20 & 0.84 & Small $(-0.06)$ \\
\hline CG2_C & $10.2(0.2)$ & $10.2(0.4)$ & -0.02 & 0.98 & Small (-0.01) \\
\hline
\end{tabular}


Figures 3 and 4 give us a better understanding of the values of the P2P_ML COP excursion on the complaint and fixed surfaces respectively. These figures demonstrate the increasing values of COP excursion in ML direction as the tasks get challenging from single task to dual-task with increasing difficulties. Figure 2 demonstrated that the TD group performed the EC, VM and CG2 tasks on the fixed surface with less ML COP excursion as compared to the CP group.

\section{Figure 3: Bar chart for the ML P2P COP Excursion for all tasks performed on Sponge Surface.}

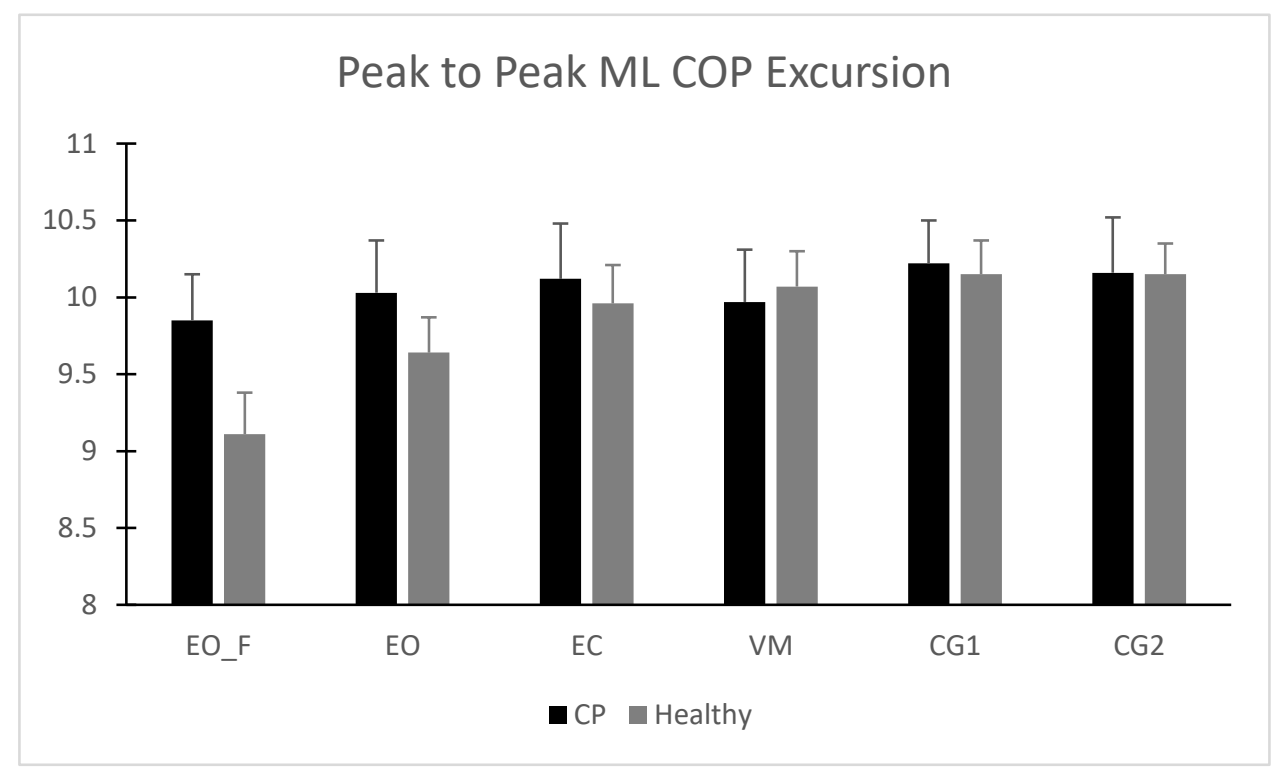

Figure 3 demonstrates the P2P COP displacement in $\mathrm{ML}$ direction for all tasks performed on the compliant surface for both the groups where Series 1 is P2P_ML COP displacement for CP (Black Color) and Series 2 for TD (Grey Color). The Y-axis consists of the P2P_ML COP displacement values and the Xaxis demonstrates the 6 tasks i.e. EO_F - Eyes Open on Fixed Surface (for reference), EO - Eyes Open, EC - Eyes Closed, VM - Visuo-Motor Tracking, CG1 - Easy Cognitive Game, CG2 - Difficult Cognitive game. 
Figure 4: Bar chart for the ML P2P COP Excursion for all tasks performed on Fixed Surface.

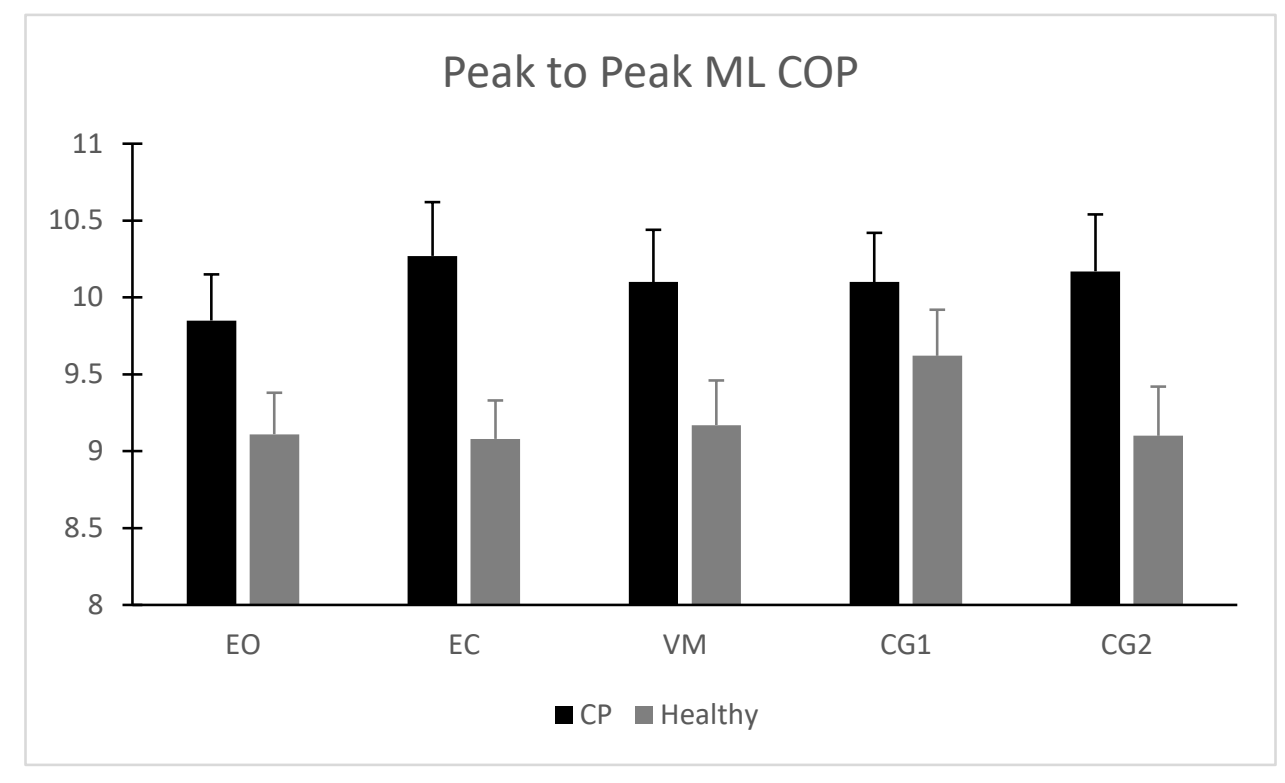

Figure 4 demonstrates the TPL for all tasks performed on the fixed surface for both the groups where Series 1 is P2P_ML COP displacement for CP (Black Color) and Series 2 for TD (Grey Color). The Y-axis consists the P2P_ML COP displacement values and the $\mathrm{X}$-axis demonstrates the 5 tasks i.e. EO - Eyes Open, EC - Eyes Closed, VM - Visuo-Motor Tracking, CG1 - Easy Cognitive Game, CG2 - Difficult Cognitive game. 
Table 4 presents the results of the unpaired t-test of the peak-to-peak $(\mathrm{P} 2 \mathrm{P})$ excursion of the COP in the Antero-posterior (AP) direction. Unexpectedly the results are all statistically insignificant, but as seen with the TPL results, the relationship between the TD and CP groups is maintained in a similar fashion. Whereas on a fixed surface, the TD group demonstrated less COP excursion in AP direction as compared to the CP group and on the contrary, the CP group demonstrated less COP excursion in AP direction on the complaint (sponge) surface. The effect sizes range from small to medium for all the tasks.

Table 4: Results of Unpaired T-test for Peak-to-Peak COP Excursion in Antero-Posterior direction (P2P_AP):

\begin{tabular}{|c|c|c|c|c|c|}
\hline Task & $\begin{array}{c}\text { TD Group } \\
\text { Mean (SEM) }\end{array}$ & $\begin{array}{c}\text { CP Group } \\
\text { Mean (SEM) }\end{array}$ & T-statistics & $\begin{array}{c}\text { Significance } \\
\text { (2-tailed) }\end{array}$ & $\begin{array}{c}\text { Effect Size } \\
\text { (Cohen's d) }\end{array}$ \\
\hline EO_F & $13.6(0.5)$ & $14.0(0.6)$ & -0.55 & 0.59 & Small (-0.16) \\
\hline EC_F & $13.27(0.5)$ & $14.6(0.7)$ & -1.51 & 0.14 & Medium (-0.43) \\
\hline VM_F & $13.2(0.5)$ & $13.5(0.5)$ & -0.43 & 0.67 & Small (-0.12) \\
\hline CG1_F & $13.5(0.5)$ & $14.0(0.6)$ & -0.62 & 0.54 & Small (-0.18) \\
\hline CG2_F & $13.0(0.5)$ & $14.3(0.71)$ & -1.38 & 0.17 & Medium (-0.39) \\
\hline EO_C & $14.4(0.4)$ & $14.7(0.3)$ & -0.66 & 0.51 & Small (-0.19) \\
\hline EC_C & $14.6(0.4)$ & $14.2(0.4)$ & 0.74 & 0.46 & Medium (0.21) \\
\hline VM_C & $15.0(0.4)$ & $14.2(0.5)$ & 1.16 & 0.25 & Medium (0.33) \\
\hline CG1_C & $15.0(0.4)$ & $14.9(0.5)$ & 0.14 & 0.89 & Small (0.04) \\
\hline CG2_C & $14.9(0.4)$ & $14.4(0.4)$ & 0.85 & 0.40 & Medium (0.24) \\
\hline
\end{tabular}


Figures 5 and 6 demonstrate the values of peak-to-peak COP excursion in anteroposterior (AP) direction for all tasks on the complaint and the fixed surfaces respectively. The increasing values of the P2P_AP excursion of the COP as the difficulty level increased with each task on both surfaces is noted for both groups.

Figure 5: Bar chart for the AP P2P COP Excursion for all tasks performed on Sponge Surface.

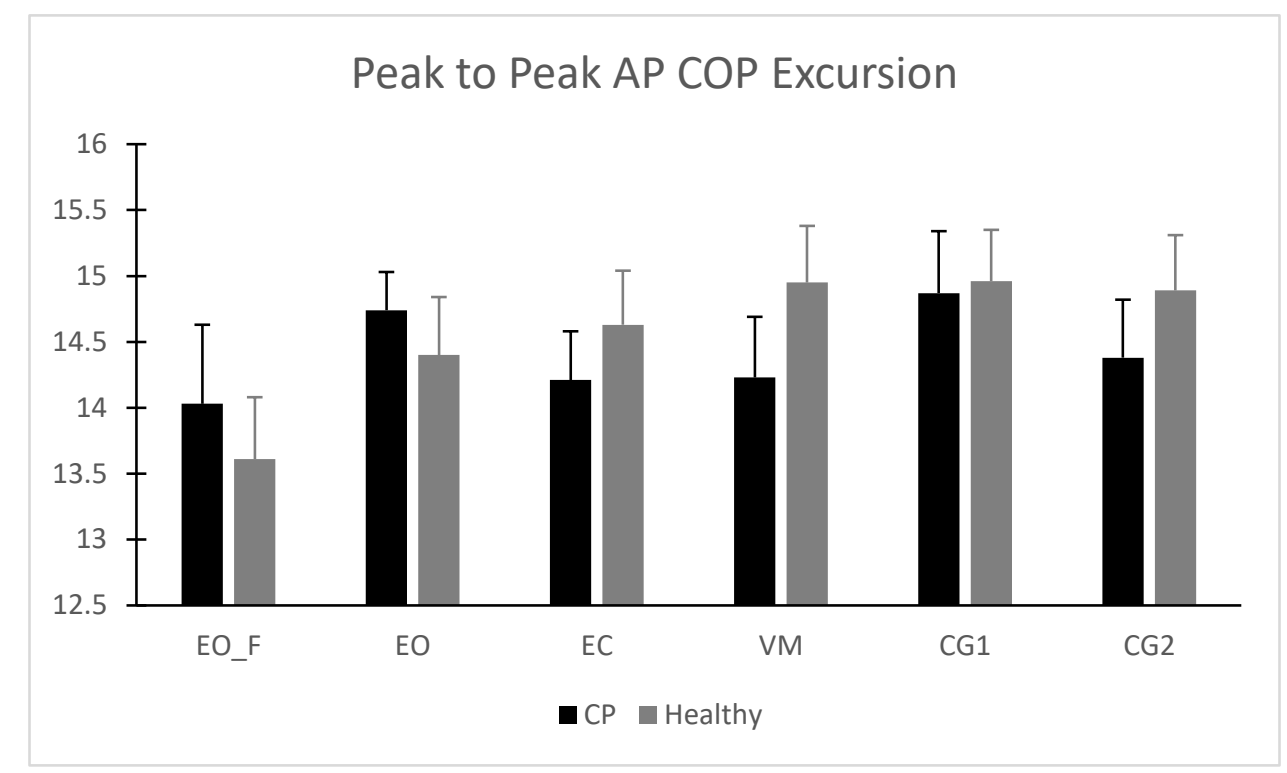

Figure 5 demonstrates the P2P COP displacement in AP direction for all tasks performed on the compliant surface for both the groups where Series 1 is P2P_AP COP displacement for CP (Black Color) and Series 2 for TD (Grey Color). The Y-axis consists of the P2P_AP COP displacement values and the Xaxis demonstrates the 6 tasks i.e. EO_F - Eyes Open on Fixed Surface (for reference), EO - Eyes Open, EC - Eyes Closed, VM - Visuo-Motor Tracking, CG1 - Easy Cognitive Game, CG2 - Difficult Cognitive game. 
Figure 6: Bar chart for the AP P2P COP Excursion for all tasks performed on Fixed Surface.

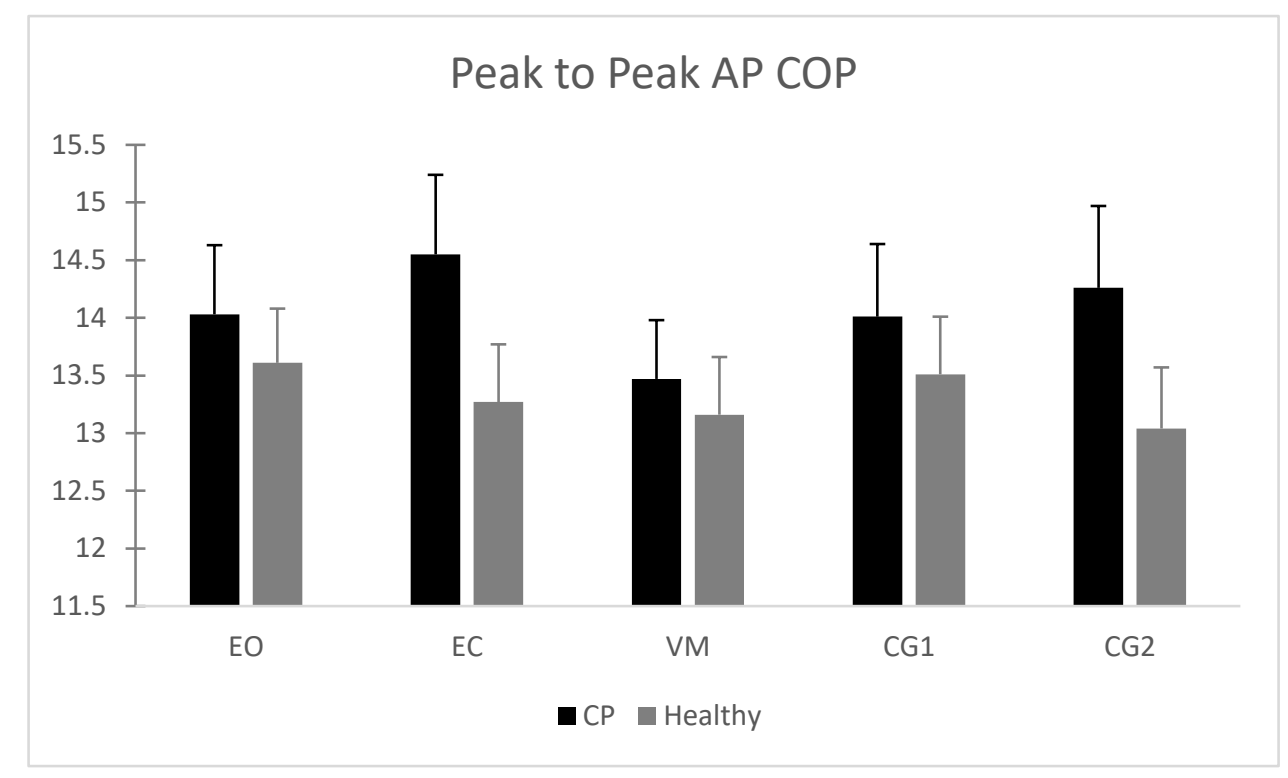

Figure 6 demonstrates the P2P COP displacement in AP direction for all tasks performed on the fixed surface for both the groups where Series 1 is P2P_AP COP displacement for CP (Black Color) and Series 2 for TD (Grey Color). The Y-axis consists the P2P_AP COP displacement values and the $X$-axis demonstrates the 5 tasks i.e. EO - Eyes Open, EC - Eyes Closed, VM - Visuo-Motor Tracking, CG1 - Easy Cognitive Game, CG2 - Difficult Cognitive game. 
Table 5 presents the results of the frequency analysis of the engagement of participants while performing the VM tracking task on both surfaces. The engagement analysis was performed by observing individual tracking game plots of each participant in both groups. If the peak-to-peak amplitude of the tracking response of a participant was less than $25 \%$ of the computer-generated response of horizontal tracking, (see figure 7) then it is considered as not performing the VM tracking task. When all 50 participants' files were observed, the TD group $(n=25)$ participants were all observed to be engaged in VM tracking tasks on both surfaces. On the other hand, it was observed that only 12 out of 25 participants in the CP group were involved in VM tracking task performance on the fixed surface and only 11 out of 25 participants were involved in VM tracking task on the compliant surface. Hence, the TD group was observed to be $100 \%$ engaged as compared to a less than $50 \%$ engagement in CP group.

\section{Figure 7: Plots of VM Tracking performance engagement levels:}

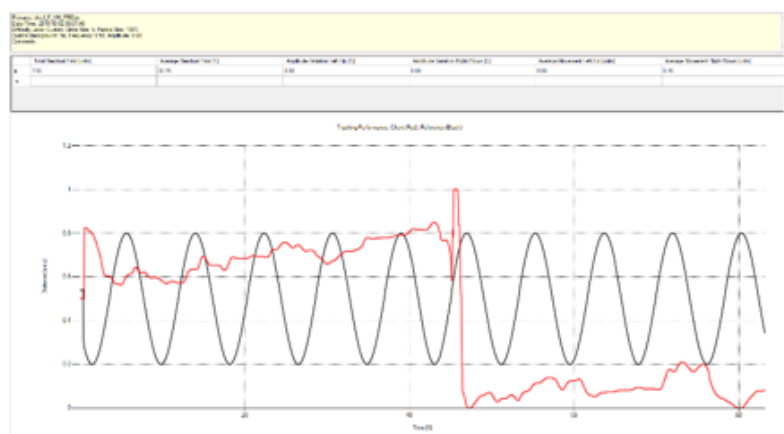

Example of VM Tracking Performance of disengaged participant

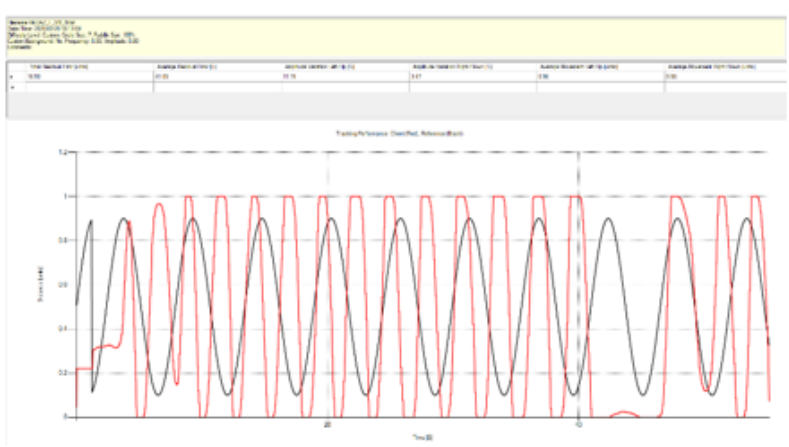

Example of VM Tracking Performance of engaged participant

Figure 7 demonstrates plots of the VM Tracking game performances. The plot on the left-hand side demonstrates the VM tracking performance of a disengaged participant whereas the plot on the righthand side denotes the VM tracking performance of an engaged participant. 
Table 5: Engagement Frequency Analysis for Visuo-Motor Tracking Task:

\begin{tabular}{|c|c|c|c|c|c|}
\hline Task & $\begin{array}{c}\text { \# of } \\
\text { Participants } \\
\text { Engaged in } \\
\text { TD group }\end{array}$ & $\begin{array}{c}\text { \% of Group } \\
\text { engagement } \\
\text { in TD group }\end{array}$ & $\begin{array}{c}\text { \# of } \\
\text { Participants } \\
\text { Engaged in } \\
\text { CP Group }\end{array}$ & $\begin{array}{c}\text { \% of Group } \\
\text { engagement } \\
\text { in CP Group }\end{array}$ & $\begin{array}{c}\text { Group \% } \\
\text { Engagement } \\
\text { Difference }\end{array}$ \\
\hline VM_F & 25 & $100 \%$ & 12 & $48 \%$ & $52 \%$ \\
\hline VM_C & 25 & $100 \%$ & 11 & $44 \%$ & $56 \%$ \\
\hline
\end{tabular}


Tables 6 and 7 present the cognitive target game data results for both groups on both surfaces. The results were all statistically significant for both the success rate and movement error. In addition, the results demonstrated a better performance by the TD group as compared to the $\mathrm{CP}$ group i.e. for $\mathrm{SR}$, the TD group showed higher values than the $\mathrm{CP}$ group and $\mathrm{ME}$ was higher in the CP group than the TD group. All game tasks demonstrated large effect sizes for both the SR and ME on both surfaces, except the ME for the CG2 task on the fixed surface demonstrated a medium effect size.

Table 6: Results of Unpaired T-test of Success Rate (SR) for Cognitive Game Tasks:

\begin{tabular}{|c|c|c|c|c|c|}
\hline Task & $\begin{array}{c}\text { TD Group } \\
\text { Mean (SEM) }\end{array}$ & $\begin{array}{c}\text { CP Group } \\
\text { Mean (SEM) }\end{array}$ & T-statistics & $\begin{array}{c}\text { Significance } \\
\text { (2-tailed) }\end{array}$ & $\begin{array}{c}\text { Effect Size } \\
\text { (Cohen's d) }\end{array}$ \\
\hline CG1_F & $61.6(5.3)$ & $31.3(6.2)$ & 3.71 & $\mathbf{0 . 0 0}$ & Large (1.05) \\
\hline CG1_C & $65.9(5.5)$ & $28.2(6.3)$ & 4.52 & $\mathbf{0 . 0 0}$ & Large (1.28) \\
\hline CG2_F & $49.7(6.8)$ & $20.5(4.4)$ & 3.63 & $\mathbf{0 . 0 0}$ & Large (1.03) \\
\hline CG2_C & $45.5(5.2)$ & $23.8(5.6)$ & 2.83 & $\mathbf{0 . 0 1}$ & Large (0.80) \\
\hline
\end{tabular}

Table 7: Results of Unpaired T-test of Movement Error (ME) for Cognitive Game Tasks:

\begin{tabular}{|c|c|c|c|c|c|}
\hline Task & $\begin{array}{c}\text { TD Group } \\
\text { Mean (SEM) }\end{array}$ & $\begin{array}{c}\text { CP Group } \\
\text { Mean (SEM) }\end{array}$ & T-statistics & $\begin{array}{c}\text { Significance } \\
\text { (2-tailed) }\end{array}$ & $\begin{array}{c}\text { Effect Size } \\
\text { (Cohen's d) }\end{array}$ \\
\hline CG1_F & $0.1(0.02)$ & $0.2(0.03)$ & -4.22 & $\mathbf{0 . 0 0}$ & Large (-1.19) \\
\hline CG1_C & $0.1(0.02)$ & $0.2(0.03)$ & -4.87 & $\mathbf{0 . 0 0}$ & Large $(-1.38)$ \\
\hline CG2_F & $0.2(0.03)$ & $0.3(0.03)$ & -2.37 & $\mathbf{0 . 0 2}$ & Medium $(-0.67)$ \\
\hline CG2_C & $0.2(0.02)$ & $0.3(0.03)$ & -3.13 & $\mathbf{0 . 0 0}$ & Large $(-0.88)$ \\
\hline
\end{tabular}


Figures 8 (SR data representation) and 9 (ME data representation) visually demonstrate the tendency of game performances for both the groups on fixed and compliant (sponge) surfaces. On observation of these figures, one can note that the success rates (SR) were higher in the TD group for all tasks in comparison with the $\mathrm{CP}$ group. Whereas, the movement error (ME) was constantly observed to be higher for the $\mathrm{CP}$ group as compared to the TD group.

\section{Figure 8: Bar chart for the Success Rate for all game tasks performed on Fixed \& Sponge Surface.}

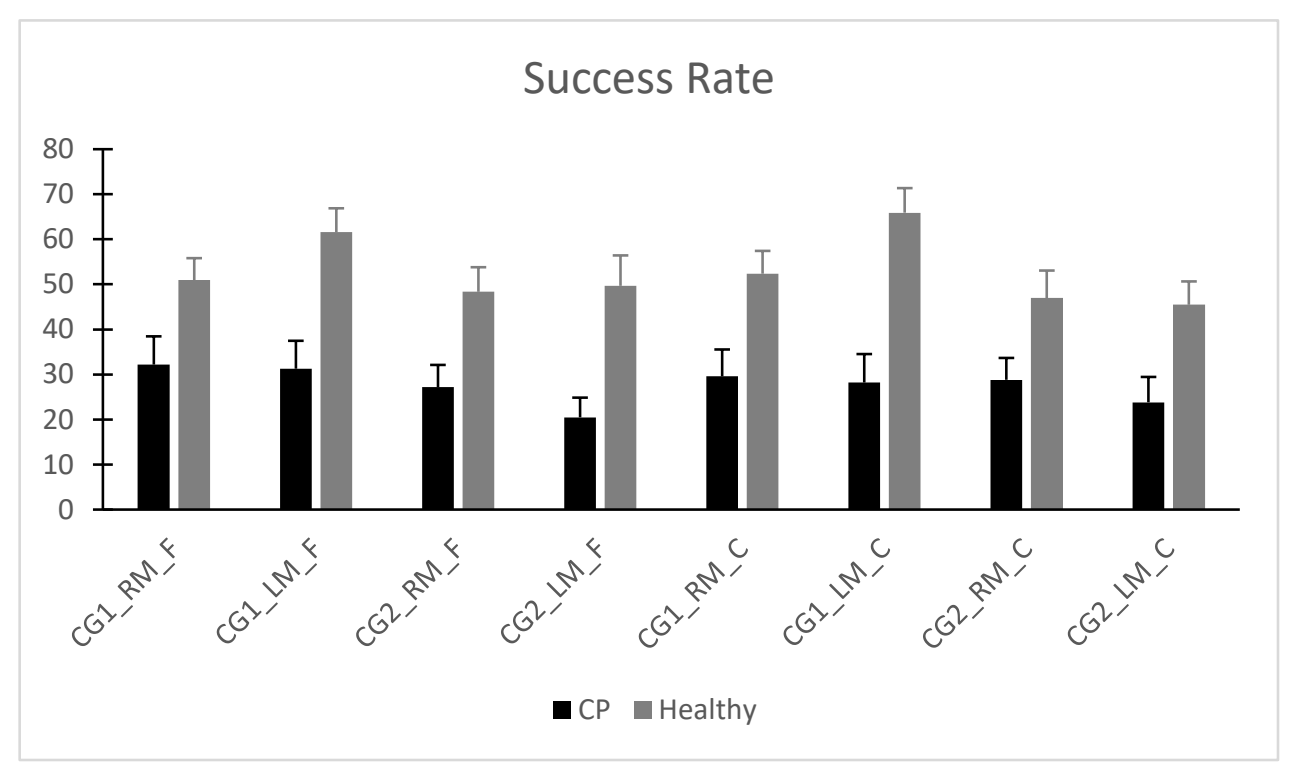

Figure 8 demonstrates the success rates (SR) for all the game tasks performed on the fixed surface for both the groups where Series 1 is SR for CP (Black Color) and Series 2 for TD (Grey Color). The Y-axis consists the $S R$ values in percentage and the $X$-axis demonstrates the success rates for 2 directions i.e. _RM - Right medium and_LM - left medium, for 2 difficulty levels of cognitive game i.e. CG1 - Easy Cognitive Game \& CG2 - Difficult Cognitive game tasks performed on the 2 surfaces i.e._F - fixed and _C - compliant surfaces. 


\section{Figure 9: Bar chart for the Movement Error for all game tasks performed on Fixed \& Sponge Surface.}

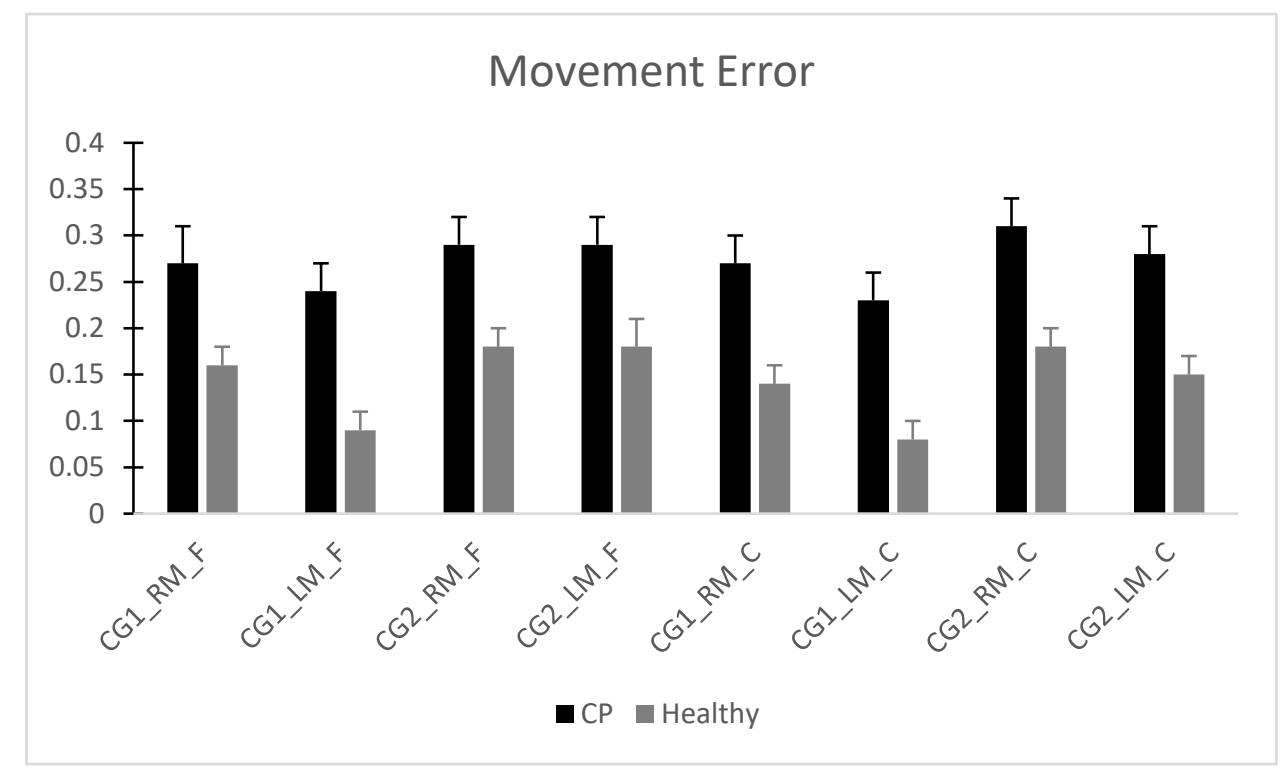

Figure 9 demonstrates the movement error $(\mathrm{ME})$ for all the game tasks performed on the fixed surface for both the groups where Series 1 is SR for CP (Black Color) and Series 2 for TD (Grey Color). The Y-axis consists the ME values and the $\mathrm{X}$-axis demonstrates the success rates for 2 directions i.e._RM - Right medium and_LM - left medium, for 2 difficulty levels of cognitive game i.e. CG1 - Easy Cognitive Game \& CG2 - Difficult Cognitive game tasks performed on the 2 surfaces i.e. _ F - fixed and _C - compliant surfaces. 
Tables 8 and 9 present the paired t-test results for the comparison of task differences on the compliant surface. Table 8 presents the task differences in the TD group. The TD group demonstrated a significant difference in the TPL as the task difficulty increased from EO single task to vision eliminated task. The difference was observed even with the addition of VM tracking and cognitive gaming with increasing difficulty levels i.e. by the addition of dualtasking. All the levels of increasing difficulties resulted in an increased TPL for TD group.

Table 8: Results of Paired Samples T-test for Comparison of task differences in the TD group:

\begin{tabular}{|c|c|c|c|c|}
\hline Pairs & $\begin{array}{c}\text { Paired Differences } \\
\text { Mean (SEM) }\end{array}$ & t-value & $\begin{array}{c}\text { Sig. (2- } \\
\text { tailed) }\end{array}$ & $\begin{array}{c}\text { Effect Size } \\
\text { (Cohen's d) }\end{array}$ \\
\hline EO - VM & $-9.5(2.79)$ & -3.42 & $\mathbf{0 . 0 0 2}$ & Medium (-0.68) \\
\hline EO - CG1 & $-35.9(4.29)$ & -8.35 & $\mathbf{0}$ & Large $(-1.67)$ \\
\hline EO - CG2 & $-33.3(4.97)$ & -6.71 & $\mathbf{0}$ & Large $(-1.34)$ \\
\hline EO - EC & $-7.6(2.03)$ & -3.72 & $\mathbf{0 . 0 0 1}$ & Medium $(-0.75)$ \\
\hline
\end{tabular}


Table 9 presents the effects of task differences in the $\mathrm{CP}$ group. The $\mathrm{CP}$ group demonstrated a statistically significant difference between the EO and CG1 and EO and CG2 tasks. The TPL increased when the difficulty level was increased by the addition of cognitive gaming i.e. dual-tasking. However, no difference was observed between EO and VM tracking tasks or EO and EC tasks performed on the compliant surface.

Table 9: Results of Paired Samples T-test for Comparison of task differences in the CP group

\begin{tabular}{|c|c|c|c|c|}
\hline Pairs & $\begin{array}{c}\text { Paired Differences } \\
\text { Mean (SEM) }\end{array}$ & t-value & $\begin{array}{c}\text { Sig. (2- } \\
\text { tailed) }\end{array}$ & $\begin{array}{c}\text { Effect Size } \\
\text { (Cohen's d) }\end{array}$ \\
\hline EO - VM & $-0.02(2.49)$ & -0.01 & 0.99 & Small $(-0.00)$ \\
\hline EO - CG1 & $-29.7(5.34)$ & -5.57 & $\mathbf{0}$ & Large $(-1.11)$ \\
\hline EO - CG2 & $-25.9(6.67)$ & -3.88 & $\mathbf{0 . 0 0 1}$ & Medium $(-0.78)$ \\
\hline EO - EC & $0.7(2.71)$ & 0.27 & 0.787 & Small $(0.06)$ \\
\hline
\end{tabular}




\subsection{Pilot Intervention Study Results:}

Table 10 presents the demographic details of all the participants $(n=16)$ for the pilot intervention part of this study. Each group had eight participants (Experimental (XG) $n=8$ and Control (CBG) $n=8$ ) who completed the intervention of 12 weeks respectively. Both groups did not differ in demographics at baseline as the mean age of participants in the experimental group was 6.1 years and the control group was 5.8 years. The control group had four participants each who belonged to GMFCS Level 1 and 2 and the experimental group comprised of three participants each who belonged to GMFCS Level $1 \& 2$ and two participants who belonged to the GMFCS Level 3.

Table 10: Demographic Table for experimental and control group participants for the pilot intervention study:

\begin{tabular}{|c|c|c|c|c|c|c|c|}
\hline $\begin{array}{c}\text { Experimental } \\
(\mathrm{XG})\end{array}$ & $\begin{array}{c}\text { Age } \\
\text { (Years })\end{array}$ & Gender & $\begin{array}{c}\text { GMFCS } \\
\text { Level }\end{array}$ & $\begin{array}{c}\text { Control } \\
(\mathrm{CBG})\end{array}$ & $\begin{array}{c}\text { Age } \\
(\text { Years })\end{array}$ & Gender & $\begin{array}{c}\text { GMFCS } \\
\text { Level }\end{array}$ \\
\hline$(\mathrm{X} 1)$ & 7.8 & $\mathrm{M}$ & 1 & $(\mathrm{C} 1)$ & 3.2 & $\mathrm{~F}$ & 2 \\
\hline$(\mathrm{X} 2)$ & 3.2 & $\mathrm{~F}$ & 2 & $(\mathrm{C} 2)$ & 3.5 & $\mathrm{~F}$ & 1 \\
\hline$(\mathrm{X} 3)$ & 9 & $\mathrm{M}$ & 2 & $(\mathrm{C} 3)$ & 9 & $\mathrm{M}$ & 2 \\
\hline$(\mathrm{X} 4)$ & 9.2 & $\mathrm{M}$ & 1 & $(\mathrm{C} 4)$ & 4 & $\mathrm{M}$ & 1 \\
\hline$(\mathrm{X} 5)$ & 3.1 & $\mathrm{M}$ & 3 & $(\mathrm{C} 5)$ & 4.5 & $\mathrm{M}$ & 1 \\
\hline$(\mathrm{X} 6)$ & 4.11 & $\mathrm{~F}$ & 3 & $(\mathrm{C} 6)$ & 9.5 & $\mathrm{~F}$ & 1 \\
\hline$(\mathrm{X} 7)$ & 5 & $\mathrm{M}$ & 2 & $(\mathrm{C} 7)$ & 7.10 & $\mathrm{~F}$ & 2 \\
\hline$(\mathrm{X} 8)$ & 7 & $\mathrm{M}$ & 1 & $(\mathrm{C} 8)$ & 5.9 & $\mathrm{M}$ & 2 \\
\hline Group Avg. & 6.1 & $\mathrm{~F}-2$ & $1-3$ & & 5.8 & $\mathrm{~F}-4$ & $1-4$ \\
& & $\mathrm{M}-6$ & $2-3$ & & & $\mathrm{M}-4$ & $2-4$ \\
& & & $3-2$ & & & & $3-0$ \\
\hline
\end{tabular}


Table 11-16 presents the results for the pilot intervention objective of this study. Table 11 presents the results of the individual pre to post-changes of the clinical outcome measures used in this study for each participant after 12 weeks of their respective interventions. The average differences in both the groups were noted to be an improvement in all the clinical outcome measures. However, the improvement for the experimental group in the Standing subtest of the GMFM-88 was $14.9 \%$, which was 5 times better as compared to $3.2 \%$ in the control group. In addition, for the Walking, Running, \& Jumping subtest of GMFM-88, the experimental group demonstrated a $13.8 \%$ improvement post-intervention, which was 6 times better as compared to a $1.5 \%$ improvement in the control group. Moreover, the improvement in the PBS scores was $10.3 \%$ in the experimental group, which was almost 20 times more as compared to a $0.5 \%$ improvement in the control group. The post-intervention improvement for PDMS-2 outcomes for the control group was $8.4 \%$ and $6.5 \%$ for the standing and locomotion subtests respectively as compared to the $4.8 \%$ and $4.7 \%$ improvement in the experimental group post-intervention. 
Table 11: Results of Clinical Outcome Measures pre and post-intervention:

\begin{tabular}{|c|c|c|c|c|c|c|c|c|c|c|c|c|}
\hline $\mathrm{O}$ & \multicolumn{4}{|c|}{ GMFM-88 } & \multirow{2}{*}{\multicolumn{2}{|c|}{$\begin{array}{l}\text { GMFM \% } \\
\text { Overall \% }\end{array}$}} & \multicolumn{4}{|c|}{ PDMS-2 } & \multicolumn{2}{|c|}{ PBS } \\
\hline $\begin{array}{c}\text { Sub } \\
- \\
\text { test } \\
\end{array}$ & \multicolumn{2}{|c|}{ Standing } & \multicolumn{2}{|c|}{$\begin{array}{l}\text { Walking, } \\
\text { Running \& } \\
\text { Jumping }\end{array}$} & & & \multicolumn{2}{|c|}{ Stationary } & \multicolumn{2}{|c|}{ Locomotion } & \multicolumn{2}{|c|}{ Score } \\
\hline & Pre & $\begin{array}{l}\text { Post } \\
\text { (Diff) }\end{array}$ & Pre & $\begin{array}{l}\text { Post } \\
\text { (Diff) }\end{array}$ & Pre & $\begin{array}{c}\text { Post } \\
\text { (Diff) }\end{array}$ & $\begin{array}{c}\mathrm{Pr} \\
\mathrm{e}\end{array}$ & $\begin{array}{l}\text { Post } \\
\text { (Diff) }\end{array}$ & Pre & $\begin{array}{c}\text { Post } \\
\text { (Diff) }\end{array}$ & Pre & $\begin{array}{l}\text { Post } \\
\text { (Diff) }\end{array}$ \\
\hline $\mathrm{C} 1$ & 0 & $2(0 \%)$ & 0 & $\begin{array}{c}0 \\
(0 \%)\end{array}$ & $18.2 \%$ & $\begin{array}{c}35.5 \% \\
(17.4 \\
\%) \\
\end{array}$ & 26 & $\begin{array}{c}27 \\
(1.9 \%)\end{array}$ & 36 & $\begin{array}{c}48 \\
(14.3 \\
\%) \\
\end{array}$ & $\begin{array}{c}13 / 5 \\
6\end{array}$ & $\begin{array}{l}13 / 56 \\
(0 \%)\end{array}$ \\
\hline $\mathrm{C} 2$ & 20 & $\begin{array}{c}24 \\
(9.1 \%)\end{array}$ & 24 & $\begin{array}{c}27 \\
(5.9 \% \\
)\end{array}$ & $65.9 \%$ & $\begin{array}{l}72.1 \% \\
(6.2 \%)\end{array}$ & 36 & $\begin{array}{c}42 \\
(7.7 \%)\end{array}$ & 73 & $\begin{array}{c}84 \\
(7 \%)\end{array}$ & $\begin{array}{c}28 / 5 \\
6\end{array}$ & $\begin{array}{c}28 / 56 \\
(0 \%)\end{array}$ \\
\hline $\mathrm{C} 3$ & 39 & $\begin{array}{c}39 \\
(0 \%)\end{array}$ & 68 & $\begin{array}{c}68 \\
(0 \%)\end{array}$ & $98.9 \%$ & $\begin{array}{c}98.9 \% \\
(0 \%)\end{array}$ & 60 & $60(0 \%)$ & 164 & $\begin{array}{c}164 \\
(0 \%)\end{array}$ & $\begin{array}{c}39 / 5 \\
6\end{array}$ & $\begin{array}{l}42 / 56 \\
(3.7 \%)\end{array}$ \\
\hline $\mathrm{C} 4$ & 37 & $\begin{array}{c}37 \\
(0 \%) \\
\end{array}$ & 65 & $\begin{array}{c}65 \\
(0 \%) \\
\end{array}$ & $97 \%$ & $\begin{array}{c}97.0 \% \\
(0 \%)\end{array}$ & 52 & $52(0 \%)$ & 129 & $\begin{array}{c}129 \\
(0 \%)\end{array}$ & $\begin{array}{c}40 / 5 \\
6\end{array}$ & $\begin{array}{c}40 / 56 \\
(0 \%)\end{array}$ \\
\hline $\mathrm{C} 5$ & 28 & $\begin{array}{c}39 \\
(16.4 \\
\%) \\
\end{array}$ & 46 & $\begin{array}{c}52 \\
(6.1 \% \\
)\end{array}$ & $85.7 \%$ & $\begin{array}{l}94.4 \% \\
(8.7 \%)\end{array}$ & 22 & $\begin{array}{c}60 \\
(46.3 \%)\end{array}$ & 106 & $\begin{array}{c}113 \\
(3.2 \%)\end{array}$ & $\begin{array}{c}40 / 5 \\
6\end{array}$ & $\begin{array}{c}40 / 56 \\
(0 \%)\end{array}$ \\
\hline C6 & 39 & $\begin{array}{c}39 \\
(0 \%) \\
\end{array}$ & 38 & $\begin{array}{c}38 \\
(0 \%) \\
\end{array}$ & $90.5 \%$ & $\begin{array}{c}90.5 \% \\
(0 \%)\end{array}$ & 46 & $46(0 \%)$ & 135 & $\begin{array}{c}135 \\
(0 \%) \\
\end{array}$ & $\begin{array}{c}39 / 5 \\
6 \\
\end{array}$ & $\begin{array}{c}39 / 56 \\
(0 \%)\end{array}$ \\
\hline $\mathrm{C} 7$ & 6 & $6(0 \%)$ & 18 & $\begin{array}{c}18 \\
(0 \%)\end{array}$ & $61.4 \%$ & $\begin{array}{c}61.4 \% \\
(0 \%)\end{array}$ & 37 & $37(0 \%)$ & 54 & $\begin{array}{c}58 \\
(3.6 \%) \\
\end{array}$ & $\begin{array}{c}18 / 5 \\
6 \\
\end{array}$ & $\begin{array}{l}18 / 56 \\
(0 \%)\end{array}$ \\
\hline $\mathrm{C} 8$ & 39 & $\begin{array}{c}39 \\
(0 \%)\end{array}$ & 41 & $\begin{array}{c}41 \\
(0 \%)\end{array}$ & $91.4 \%$ & $\begin{array}{c}91.4 \% \\
(0 \%)\end{array}$ & 45 & $\begin{array}{c}56 \\
(10.9 \%)\end{array}$ & 114 & $\begin{array}{l}105(- \\
4.1 \%)\end{array}$ & $\begin{array}{c}40 / 5 \\
6\end{array}$ & $\begin{array}{l}40 / 56 \\
(0 \%)\end{array}$ \\
\hline Avg. & Diff & $3.2 \%$ & & $1.5 \%$ & & $4 \%$ & & $8.4 \%$ & & $6.5 \%$ & & $0.5 \%$ \\
\hline $\mathrm{X} 1$ & 39 & $\begin{array}{c}39 \\
(0 \%)\end{array}$ & 68 & $\begin{array}{c}68 \\
(0 \%) \\
\end{array}$ & $98.9 \%$ & $\begin{array}{c}98.9 \% \\
(0 \%)\end{array}$ & 60 & $60(0 \%)$ & 164 & $\begin{array}{c}164 \\
(0 \%)\end{array}$ & $\begin{array}{c}41 / 5 \\
6\end{array}$ & $\begin{array}{c}49 / 56 \\
(8.9 \%)\end{array}$ \\
\hline $\mathrm{X} 2$ & 9 & $9(0 \%)$ & 12 & $\begin{array}{c}12 \\
(0 \%)\end{array}$ & $49.5 \%$ & $\begin{array}{c}49.5 \% \\
(0 \%)\end{array}$ & 36 & $\begin{array}{c}42 \\
(7.7 \%)\end{array}$ & 62 & $\begin{array}{c}74 \\
(8.7 \%)\end{array}$ & $\begin{array}{c}13 / 5 \\
6\end{array}$ & $\begin{array}{c}17 / 56 \\
(13.3 \% \\
) \\
\end{array}$ \\
\hline $\mathrm{X} 3$ & 34 & $\begin{array}{c}37 \\
(4.2 \%)\end{array}$ & 55 & $\begin{array}{c}60 \\
(4.4 \% \\
)\end{array}$ & $92.7 \%$ & $\begin{array}{c}95.6 \% \\
(3 \%)\end{array}$ & 49 & $\begin{array}{c}54 \\
(4.9 \%)\end{array}$ & 121 & $\begin{array}{c}137 \\
(6.2 \%)\end{array}$ & $\begin{array}{c}37 / 5 \\
6\end{array}$ & $\begin{array}{l}39 / 56 \\
(2.6 \%)\end{array}$ \\
\hline $\mathrm{X} 4$ & 39 & $\begin{array}{c}39 \\
(0 \%)\end{array}$ & 68 & $\begin{array}{c}68 \\
(0 \%)\end{array}$ & $98.9 \%$ & $\begin{array}{c}98.9 \% \\
(0 \%)\end{array}$ & 60 & $60(0 \%)$ & 164 & $\begin{array}{c}164 \\
(0 \%)\end{array}$ & $\begin{array}{c}50 / 5 \\
6\end{array}$ & $\begin{array}{l}54 / 56 \\
(3.9 \%)\end{array}$ \\
\hline $\mathrm{X} 5$ & 3 & $\begin{array}{c}36 \\
(84.6 \\
\%) \\
\end{array}$ & 8 & $\begin{array}{c}18 \\
(38.5 \\
\%) \\
\end{array}$ & $60 \%$ & $\begin{array}{c}80.1 \% \\
(20.1 \\
\%) \\
\end{array}$ & 36 & $\begin{array}{c}48 \\
(14.3 \%)\end{array}$ & 64 & $\begin{array}{c}80 \\
(11.1 \\
\%) \\
\end{array}$ & $\begin{array}{c}16 / 5 \\
6\end{array}$ & $\begin{array}{c}27 / 56 \\
(25.6 \% \\
\quad) \\
\end{array}$ \\
\hline X6 & 8 & $\begin{array}{c}15 \\
(30.4 \\
\%)\end{array}$ & 6 & $\begin{array}{c}27 \\
(63.6 \\
\%) \\
\end{array}$ & $58.2 \%$ & $\begin{array}{c}84.2 \% \\
(26.1 \\
\%) \\
\end{array}$ & 38 & $38(0 \%)$ & 71 & $\begin{array}{c}71 \\
(0 \%)\end{array}$ & $\begin{array}{c}16 / 5 \\
6\end{array}$ & $\begin{array}{l}25 / 56 \\
(22 \%)\end{array}$ \\
\hline $\mathrm{X} 7$ & 6 & $6(0 \%)$ & 9 & $\begin{array}{c}9 \\
(0 \%)\end{array}$ & $46.4 \%$ & $\begin{array}{c}46.4 \% \\
(0 \%)\end{array}$ & 36 & $\begin{array}{c}40 \\
(5.3 \%) \\
\end{array}$ & 57 & $\begin{array}{c}64 \\
(5.8 \%) \\
\end{array}$ & $\begin{array}{c}11 / 5 \\
6 \\
\end{array}$ & $\begin{array}{l}11 / 56 \\
(0 \%)\end{array}$ \\
\hline
\end{tabular}




\begin{tabular}{|c|c|c|c|c|c|c|c|c|c|c|c|c|}
\hline X8 & 39 & $\begin{array}{c}39 \\
(0 \%)\end{array}$ & 51 & $\begin{array}{c}55 \\
(3.8 \% \\
)\end{array}$ & $94.2 \%$ & $\begin{array}{c}95.1 \% \\
(0.9 \%)\end{array}$ & 48 & $\begin{array}{c}54 \\
(5.9 \%)\end{array}$ & 124 & $\begin{array}{c}139 \\
(5.7 \%)\end{array}$ & $\begin{array}{c}41 / 5 \\
6\end{array}$ & $\begin{array}{c}46 / 56 \\
(5.8 \%)\end{array}$ \\
\hline Avg. Diff & $14.9 \%$ & & $\begin{array}{c}13.8 \\
\%\end{array}$ & & $6.2 \%$ & & $4.8 \%$ & & $4.7 \%$ & & $10.3 \%$ \\
\hline $\begin{array}{c}\text { CBG V/s } \\
\text { XG }\end{array}$ & $11.7 \%$ & $\begin{array}{c}- \\
12.3 \\
\%\end{array}$ & & $-2.2 \%$ & & $3.6 \%$ & & $1.8 \%$ & & $-9.8 \%$ \\
\hline
\end{tabular}

Tables $12 \& 13$ presents the results of the individual pre to post-change in the balance performances (TPL) on fixed and compliant surfaces respectively. On observing the results of all the tasks on the fixed surface (see table 12), it was noted that the COP displacements were consistently decreased after the respective intervention in both groups. Seven out of eight participants in the experimental group demonstrated a decreased TPL while performance of EO task on the fixed surface as compared to only 4 out of 8 participants in the control group. The experimental group demonstrated a $28.8 \%$ reduction in average TPL post-intervention in EO task on fixed surface, which was 12 times lower as compared to a $2.8 \%$ reduction in TPL of the control group in the same task.

Almost a 10 times lower average TPL values for EC tasks on fixed surface were noted for the experimental group (17\%) as compared to the control group (1.8\%). Moreover, 6 out of 8 participants in experimental group demonstrated a decreased TPL as compared to 5 out of 8 participants in the control group.

In addition, the COP displacement in the experimental group was decreased by $35.9 \%$ during the performance of VM tracking tasks as compared to $20.5 \%$ reduction in TPL for the control group. Seven out of eight participants in both groups demonstrated a decreased TPL postintervention. 
For the CG task on the fixed surface, all eight participants in the experimental group demonstrated a decreased TPL post-intervention as compared to 5 out of 8 participants in the control group. An average decrease of TPL for the experimental group was $36.7 \%$ as compared to a $10.5 \%$ decreased TPL for the control group for CG task performance on fixed surface. 
Table 12: Table for Pre-Post changes of Total Path Length (TPL) for tasks on Fixed Surface:

\begin{tabular}{|c|c|c|c|c|c|c|c|c|}
\hline Task & \multicolumn{2}{|c|}{ EO_F } & \multicolumn{2}{|c|}{ EC_F } & \multicolumn{2}{|r|}{ VM_F } & \multicolumn{2}{|r|}{ CG_F } \\
\hline $\begin{array}{c}\text { Assessme } \\
\text { nt Time }\end{array}$ & Pre & Post (Diff) & Pre & Post (Diff) & Pre & Post (Diff) & Pre & Post (Diff) \\
\hline $\mathrm{C} 1$ & 37.8 & $\begin{array}{c}75.9 \\
(33.5 \%)\end{array}$ & 43.3 & $\begin{array}{c}55.1 \\
(11.9 \%)\end{array}$ & 31.4 & $26.9(-7.7 \%)$ & 37.2 & $\begin{array}{c}74.7 \\
(33.5 \%)\end{array}$ \\
\hline $\mathrm{C} 2$ & 54.5 & $56.5(1.8 \%)$ & 75.6 & $\begin{array}{l}58.9(- \\
12.4 \%)\end{array}$ & 66.4 & $\begin{array}{l}27.3(- \\
41.8 \%)\end{array}$ & 91.9 & $\begin{array}{l}44.8(- \\
34.5 \%)\end{array}$ \\
\hline C3 & 19.2 & $19.2(0 \%)$ & 22.4 & $22.4(0 \%)$ & 39.5 & $\begin{array}{l}30.9(- \\
12.2 \%)\end{array}$ & 57.9 & $\begin{array}{l}31.5(- \\
29.6 \%)\end{array}$ \\
\hline $\mathrm{C} 4$ & 41.8 & $35.6(-8 \%)$ & 23.5 & $\begin{array}{c}23.8 \\
(0.8 \%)\end{array}$ & 38.9 & $\begin{array}{l}24.8(- \\
22.1 \%)\end{array}$ & 79.4 & $88.5(5.4 \%)$ \\
\hline $\mathrm{C} 5$ & 25.4 & $\begin{array}{c}31.5 \\
(10.7 \%) \\
\end{array}$ & 17.4 & $\begin{array}{c}23.6 \\
(15.1 \%) \\
\end{array}$ & 27.1 & $29.4(4.1 \%)$ & 40.8 & $36.3(-5.9 \%)$ \\
\hline C6 & 30.8 & $\begin{array}{l}25.3(- \\
9.8 \%) \\
\end{array}$ & 27.8 & $24(-7.3 \%)$ & 28.5 & $13.7(-35 \%)$ & 31.2 & $31.9(1.2 \%)$ \\
\hline $\mathrm{C} 7$ & 37.9 & $\begin{array}{l}23.6(- \\
23.3 \%) \\
\end{array}$ & 35.6 & $\begin{array}{l}25.5(- \\
16.6 \%) \\
\end{array}$ & 43.9 & $\begin{array}{l}32.6(- \\
14.8 \%) \\
\end{array}$ & 70.6 & $\begin{array}{l}50.9(- \\
16.2 \%) \\
\end{array}$ \\
\hline $\mathrm{C} 8$ & 39.1 & $45.6(7.7 \%)$ & 32 & $\begin{array}{l}28.5(- \\
5.8 \%)\end{array}$ & 78.4 & $\begin{array}{l}38.3(- \\
34.4 \%) \\
\end{array}$ & 99.2 & $\begin{array}{l}44.4(- \\
38.2 \%) \\
\end{array}$ \\
\hline Avg. Diff & & $-2.4 \%$ & & $-1.8 \%$ & & $-20.5 \%$ & & $-10.5 \%$ \\
\hline $\mathrm{X} 1$ & 44.8 & $45.1(0.3 \%)$ & 25.4 & $\begin{array}{c}52.1 \\
(34.5 \%)\end{array}$ & 62.9 & $\begin{array}{l}45.7(- \\
15.9 \%) \\
\end{array}$ & 72.8 & $\begin{array}{l}51.4(- \\
17.2 \%) \\
\end{array}$ \\
\hline $\mathrm{X} 2$ & 144 & $\begin{array}{l}35.9(- \\
60.1 \%)\end{array}$ & 180.8 & $\begin{array}{l}37.6(- \\
65.5 \%) \\
\end{array}$ & $\begin{array}{c}190 . \\
8\end{array}$ & $\begin{array}{l}32.8(- \\
70.7 \%)\end{array}$ & 154 & $\begin{array}{l}30.3(- \\
67.1 \%) \\
\end{array}$ \\
\hline $\mathrm{X} 3$ & 66.2 & $\begin{array}{l}26.8(- \\
42.3 \%) \\
\end{array}$ & 59.2 & $\begin{array}{c}38(- \\
21.8 \%) \\
\end{array}$ & 61.3 & $\begin{array}{l}23.7(- \\
44.3 \%) \\
\end{array}$ & 59.1 & $\begin{array}{l}30.9(- \\
31.4 \%) \\
\end{array}$ \\
\hline $\mathrm{X} 4$ & 19.8 & $\begin{array}{l}19.3(- \\
1.4 \%)\end{array}$ & 16.1 & $\begin{array}{c}19.5 \\
(9.8 \%)\end{array}$ & 14.4 & $18.9(13.3 \%)$ & 25.4 & $24.7(-1.3 \%)$ \\
\hline $\mathrm{X} 5$ & 104.7 & $\begin{array}{l}41.9(- \\
42.8 \%)\end{array}$ & LOB & 35.6 (NA) & $\begin{array}{c}151 . \\
2\end{array}$ & $\begin{array}{l}25.9(- \\
70.8 \%)\end{array}$ & $\begin{array}{c}155 . \\
8\end{array}$ & $\begin{array}{l}50.3(- \\
51.2 \%)\end{array}$ \\
\hline X6 & 78.9 & $\begin{array}{l}32.8(- \\
41.4 \%) \\
\end{array}$ & 79.2 & $\begin{array}{l}24.8(- \\
52.3 \%) \\
\end{array}$ & $\begin{array}{c}101 . \\
2\end{array}$ & $\begin{array}{l}34.8(- \\
48.9 \%) \\
\end{array}$ & $\begin{array}{c}111 . \\
1\end{array}$ & $\begin{array}{l}43.3(- \\
43.9 \%) \\
\end{array}$ \\
\hline $\mathrm{X} 7$ & 143.1 & $\begin{array}{l}74.6(- \\
31.4 \%)\end{array}$ & 101 & $\begin{array}{l}81.9(- \\
10.5 \%)\end{array}$ & $\begin{array}{c}157 . \\
4\end{array}$ & $62.7(-43 \%)$ & $\begin{array}{c}164 . \\
4\end{array}$ & $45.1(-57 \%)$ \\
\hline $\mathrm{X} 8$ & 22.9 & $\begin{array}{l}18.2(- \\
11.5 \%)\end{array}$ & 20.5 & $\begin{array}{l}15.9(- \\
12.9 \%)\end{array}$ & 38.5 & $33.5(-7.1 \%)$ & 49.9 & $\begin{array}{l}30.2(- \\
24.6 \%)\end{array}$ \\
\hline Avg. Diff & & $-28.8 \%$ & & $-17 \%$ & & $-35.9 \%$ & & $-36.7 \%$ \\
\hline $\begin{array}{c}\text { CBG V/s } \\
\text { XG }\end{array}$ & & $26.4 \%$ & & $15.2 \%$ & & $15.4 \%$ & & $26.2 \%$ \\
\hline
\end{tabular}


Table 13 demonstrates the pre to post-change of TPL for all tasks on complaint (sponge) surface for the participants in both groups. The results demonstrate that the experimental group showed a decreased TPL post-intervention as expected whereas the control group showed an increase in the TPL post-intervention in 3 out of 4 tasks.

In the experimental group, 6 out of 8 participants demonstrated a decrease in the TPL on complaint surface during the performance of EO task. Whereas, only 2 out of 8 participants demonstrated a decrease in TPL post intervention while performance of EO task on compliant surface in the control group. The experimental group demonstrated a $13.3 \%$ decrease in TPL for the EO task post-intervention as compared to an $8.5 \%$ increase in the control group.

On the other hand, for the EC task on the complaint surface, 4 out of 8 participants in the experimental group demonstrated a decrease in TPL post intervention as compared to only 1 out of 8 participants in the control group. An average decrease of 5.3\% was noted in the EC task on the compliant surface in the experimental group whereas the control group demonstrated an increase of $6.8 \%$.

During performance of VM Tracking task on the complaint surface, 6 out of 8 participants in the experimental group demonstrated a decrease in TPL post-intervention as compared to only 3 out of 8 participants in the control group. The experimental group demonstrated an average decrease of $9 \%$ in TPL during VM tracking task performance on the compliant surface; on the other hand, an $8.1 \%$ increase was noted in the control group TPL.

Both groups demonstrated a decrease in the TPL during the performance of CG task with the magnitudes of $18.4 \%$ decrease in TPL of the experimental group and a $13.7 \%$ decrease in the 
TPL of the control group. Seven out of eight participants demonstrated a decrease in TPL during the CG task performance on the complaint surface in both groups.

Table 13: Table for Pre-Post changes of Total Path Length (TPL) for tasks on Compliant Surface:

\begin{tabular}{|c|c|c|c|c|c|c|c|c|}
\hline Task & \multicolumn{2}{|c|}{ EO_C } & \multicolumn{2}{|c|}{ EC_C } & \multicolumn{2}{|r|}{ VM_C } & \multicolumn{2}{|c|}{ CG_C } \\
\hline $\begin{array}{c}\text { Assessmen } \\
\text { t Time }\end{array}$ & Pre & $\begin{array}{l}\text { Post } \\
\text { (Diff) }\end{array}$ & Pre & $\begin{array}{l}\text { Post } \\
\text { (Diff) }\end{array}$ & Pre & $\begin{array}{l}\text { Post } \\
\text { (Diff) }\end{array}$ & Pre & $\begin{array}{c}\text { Post } \\
\text { (Diff) }\end{array}$ \\
\hline $\mathrm{C} 1$ & 34.3 & $\begin{array}{c}42.9 \\
(11.2 \%)\end{array}$ & 35.5 & $\begin{array}{c}36 \\
(0.7 \%)\end{array}$ & 27.7 & $35(11.7 \%)$ & 55.9 & $\begin{array}{l}39.2(- \\
17.6 \%)\end{array}$ \\
\hline $\mathrm{C} 2$ & 34.9 & $\begin{array}{c}48.3 \\
(16.1 \%) \\
\end{array}$ & 46.2 & $\begin{array}{c}49.3 \\
(3.3 \%) \\
\end{array}$ & 24.4 & $49.1(33.7 \%)$ & 38.3 & $\begin{array}{c}54.6 \\
(17.5 \%) \\
\end{array}$ \\
\hline $\mathrm{C} 3$ & 22.3 & $\begin{array}{c}25.3 \\
(6.4 \%)\end{array}$ & 34.1 & $\begin{array}{l}29.9(- \\
6.6 \%)\end{array}$ & 28.7 & $23.7(-9.6 \%)$ & 39.4 & $\begin{array}{l}22.5(- \\
27.3 \%)\end{array}$ \\
\hline $\mathrm{C} 4$ & 42.1 & $\begin{array}{c}44.4 \\
(2.7 \%)\end{array}$ & 22.6 & $\begin{array}{c}38.4 \\
(26 \%)\end{array}$ & 32.8 & $40.2(10.1 \%)$ & 51.2 & $\begin{array}{c}41(- \\
11.1 \%)\end{array}$ \\
\hline $\mathrm{C} 5$ & 25.6 & $\begin{array}{c}71.5 \\
(47.3 \%)\end{array}$ & 21.3 & $\begin{array}{c}28.1 \\
(13.6 \%)\end{array}$ & 30.5 & $30.4(-0.2 \%)$ & 40.1 & $\begin{array}{l}32.7(- \\
10.1 \%)\end{array}$ \\
\hline C6 & 43.6 & $\begin{array}{l}33.5(- \\
13.1 \%)\end{array}$ & 31.5 & $\begin{array}{c}32.4 \\
(1.4 \%)\end{array}$ & 41.9 & $\begin{array}{l}22.47(- \\
27.4 \%)\end{array}$ & 57.8 & $\begin{array}{l}42.9(- \\
14.8 \%)\end{array}$ \\
\hline $\mathrm{C} 7$ & 55 & $\begin{array}{c}61.2 \\
(5.4 \%) \\
\end{array}$ & 27.5 & $\begin{array}{c}33.6 \\
(10 \%)\end{array}$ & 52.7 & $61.4(40.2 \%)$ & 69.1 & $\begin{array}{c}43(- \\
23.2 \%) \\
\end{array}$ \\
\hline $\mathrm{C} 8$ & 78.6 & $\begin{array}{l}67.5(- \\
7.6 \%)\end{array}$ & 62.8 & $\begin{array}{c}70.4 \\
(5.7 \%)\end{array}$ & 54.4 & $68.7(6 \%)$ & 112.8 & $\begin{array}{l}70.8(- \\
22.9 \%) \\
\end{array}$ \\
\hline Avg. Diff & & $8.5 \%$ & & $6.8 \%$ & & $8.1 \%$ & & $-13.7 \%$ \\
\hline $\mathrm{X} 1$ & 37.7 & $\begin{array}{c}30.3(- \\
11 \%)\end{array}$ & 28.3 & $\begin{array}{l}43.4 \\
(21 \%)\end{array}$ & 23 & $37.2(23.7 \%)$ & 36.4 & $\begin{array}{l}35.7(- \\
1.1 \%)\end{array}$ \\
\hline $\mathrm{X} 2$ & 79.8 & $\begin{array}{c}31.8(- \\
43 \%)\end{array}$ & 81.9 & $\begin{array}{l}38.1(- \\
36.5 \%)\end{array}$ & 100.4 & $50.9(-32.7 \%)$ & 69.5 & $\begin{array}{l}47.1(- \\
19.2 \%)\end{array}$ \\
\hline $\mathrm{X} 3$ & 42.3 & $\begin{array}{l}27.5(- \\
21.2 \%)\end{array}$ & 48 & $\begin{array}{l}32(- \\
20 \%)\end{array}$ & 53.8 & $27(-33.2 \%)$ & 48.8 & $\begin{array}{l}24.3(- \\
33.6 \%)\end{array}$ \\
\hline $\mathrm{X} 4$ & 31.5 & $\begin{array}{l}26.3(- \\
8.9 \%)\end{array}$ & 28.5 & $\begin{array}{c}29.7 \\
(2.1 \%)\end{array}$ & 27.5 & $26.7(-1.5 \%)$ & 51.3 & $\begin{array}{l}21.8(- \\
40.4 \%)\end{array}$ \\
\hline $\mathrm{X} 5$ & 53.4 & $\begin{array}{l}38.2(- \\
16.6 \%)\end{array}$ & 42.1 & $\begin{array}{c}48.8 \\
(7.3 \%)\end{array}$ & 57.6 & $37.1(21.7 \%)$ & 50.8 & $\begin{array}{l}34.9(- \\
18.6 \%)\end{array}$ \\
\hline X6 & 38.1 & $\begin{array}{c}39.2 \\
(1.4 \%)\end{array}$ & 50.5 & $\begin{array}{l}35.6(- \\
17.3 \%) \\
\end{array}$ & 37 & $36.9(-0.2 \%)$ & 49.7 & $\begin{array}{l}35.9(- \\
16.1 \%) \\
\end{array}$ \\
\hline $\mathrm{X} 7$ & 87.9 & $\begin{array}{c}91.7 \\
(2.1 \%)\end{array}$ & 103.8 & $\begin{array}{l}85.6(- \\
9.6 \%) \\
\end{array}$ & 114.7 & $\begin{array}{l}79.50(- \\
18.1 \%) \\
\end{array}$ & 86.8 & $92.1(3 \%)$ \\
\hline $\mathrm{X} 8$ & 45 & $37.6(-9 \%)$ & 39.8 & $\begin{array}{c}49.4 \\
(10.7 \%) \\
\end{array}$ & 56.6 & $71.5(11.6 \%)$ & 80 & $\begin{array}{l}52.4(- \\
20.9 \%) \\
\end{array}$ \\
\hline Avg. Diff & & $-13.3 \%$ & & $-5.3 \%$ & & $-9 \%$ & & $-18.4 \%$ \\
\hline $\begin{array}{c}\text { CBG V/s } \\
\text { XG }\end{array}$ & & $-21.8 \%$ & & $-12.1 \%$ & & $-17.1 \%$ & & $-4.7 \%$ \\
\hline
\end{tabular}


Table 14 presents the outcome of the Visuomotor Tracking task on both surfaces in each group. The average residual error (ARE) decreased in both groups on the fixed surface. On the fixed surface, 7 out of 8 participants in the experimental group demonstrated a decreased ARE whereas only 5 out of 8 participants in the control group demonstrated a decrease in ARE postintervention. The average ARE decrease in the experimental group was $4.1 \%$ as compared to a $3.3 \%$ in the control group.

On the other hand, 6 out of 8 participants in the experimental group demonstrated a decrease in ARE on the compliant surface and only 3 out of 8 participants in the control group demonstrated a decrease in ARE post-intervention. A 0\% average decrease in ARE on the compliant (sponge) surface was noted in the control group post 12 weeks of intervention, and a 3.6\% average decrease in ARE on the compliant (sponge) surface in the experimental group was observed post 12 weeks. 
Table 14: Table for Pre-Post changes of Average Residual Error (ARE) for Visuo-Motor Tracking performance:

\begin{tabular}{|c|c|c|c|c|}
\hline Outcome Measure & \multicolumn{2}{|c|}{ ARE_F } & \multicolumn{2}{c|}{ ARE_C } \\
\hline Participants & Pre & Post (Diff) & Pre & Post (Diff) \\
\hline C1 & 33.3 & $29.4(-6.1 \%)$ & 35 & $30.9(-6.2 \%)$ \\
\hline C2 & 35 & $35.1(0 \%)$ & 32.9 & $31.9(-1.5 \%)$ \\
\hline C3 & 31.8 & $22.9(-16.2 \%)$ & 38.8 & $39.8(1.3 \%)$ \\
\hline C4 & 28.2 & $27.7(-0.8 \%)$ & 23.1 & $23.1(0 \%)$ \\
\hline C5 & 28.5 & $28.8(0.6 \%)$ & 37.9 & $39.2(1.7 \%)$ \\
\hline C6 & 41.4 & $39(-3 \%)$ & 38.6 & $33.8(-6.6 \%)$ \\
\hline C7 & 42.1 & $50.1(8.7 \%)$ & 33.4 & $34.5(1.6 \%)$ \\
\hline C8 & 37.1 & $30.6(-9.6 \%)$ & 20.3 & $24.8(9.9 \%)$ \\
\hline Avg. Diff. & & $-3.3 \%$ & & $0 \%$ \\
\hline X1 & 23.8 & $17.3(-15.7 \%)$ & 25.4 & $29.4(7.3 \%)$ \\
\hline X2 & 35.2 & $30.2(-7.7 \%)$ & 37.3 & $34.8(-3.5 \%)$ \\
\hline X3 & 19 & $19(-0.1 \%)$ & 28.2 & $23.2(-9.8 \%)$ \\
\hline X4 & 12.8 & $11.9(-3.6 \%)$ & 16.2 & $13.4(-9.6 \%)$ \\
\hline X5 & 22.9 & $22.1(-1.9 \%)$ & 37.4 & $40.8(4.5 \%)$ \\
\hline X6 & 26.4 & $23.2(-6.4 \%)$ & 37.4 & $33.4(-5.7 \%)$ \\
\hline X7 & 20.1 & $22.7(6.1 \%)$ & 38 & $35.1(-4.1 \%)$ \\
\hline X8 & 21.5 & $20.2(-3.2 \%)$ & 31.5 & $27.1(-7.6 \%)$ \\
\hline Avg. Diff. & & $-4.1 \%$ & & $-3.6 \%$ \\
\hline CBG V/s XG & & $-0.8 \%$ & & $-3.6 \%$ \\
\hline
\end{tabular}


Tables $15 \& 16$ summarizes the cognitive game performance changes in both groups on both surfaces. Table 15 presents the results of the SR for the game tasks on fixed and compliant (sponge) surfaces for both groups. All participants demonstrated an increase in post-intervention SR in both groups. An average increase of $27-31 \%$ in SR of both groups were noted for all the cognitive game tasks.

Table 15: Results for Pre-Post changes of Success Rate for Cognitive Target Game:

\begin{tabular}{|c|c|c|c|c|}
\hline CG task & \multicolumn{2}{|c|}{ CG_F } & \multicolumn{2}{c|}{ CG_C } \\
\hline OM & \multicolumn{2}{|c|}{ SR } & \multicolumn{2}{c|}{ SR } \\
\hline & Pre & Post (Diff) & Pre & Post (Diff) \\
\hline C1 & 0 & $75(75 \%)$ & 0 & $0(0 \%)$ \\
\hline C2 & 0 & $60(60 \%)$ & 0 & $80(80 \%)$ \\
\hline C3 & 0 & $33.3(33.3 \%)$ & 75 & $80(5 \%)$ \\
\hline C4 & 0 & $27.3(27.3 \%)$ & 0 & $28.6(28.6 \%)$ \\
\hline C5 & 0 & $14.3(14.3 \%)$ & 0 & $0(0 \%)$ \\
\hline C6 & 25 & $28.6(3.6 \%)$ & 33.3 & $100(66.7 \%)$ \\
\hline C7 & 0 & $0(0 \%)$ & 0 & $0(0 \%)$ \\
\hline C8 & 50 & $60(10 \%)$ & 0 & $71.4(71.4 \%)$ \\
\hline Avg. Diff & & $27.9 \%$ & & $31.5 \%$ \\
\hline X1 & 40 & $66.7(26.7 \%)$ & 100 & NA \\
\hline X2 & 0 & $33.3(33.3 \%)$ & 25 & $50.00(25 \%)$ \\
\hline X3 & 16.7 & $66.7(50 \%)$ & 50 & $60.00(10 \%)$ \\
\hline X4 & 40 & $66.7(26.7 \%)$ & 42.9 & $40.00(-2.9 \%)$ \\
\hline X5 & 0 & $50(50 \%)$ & 33.3 & $75.00(41.7 \%)$ \\
\hline X6 & 0 & $25(25 \%)$ & 16.7 & $40.00(23.3 \%)$ \\
\hline X7 & 0 & $25(25 \%)$ & 0 & $66.67(66.7 \%)$ \\
\hline X8 & 16.7 & $33.3(16.7 \%)$ & 0 & $37.50(37.5 \%)$ \\
\hline Avg. Diff & & $31.7 \%$ & & $28.8 \%$ \\
\hline CBG V/s & & $-3.7 \%$ & & $2.7 \%$ \\
\hline XG & & & & \\
\hline & & & & \\
\hline
\end{tabular}


On the other hand, a $12 \%$ increase (instead of a decrease) in the ME for the control group was noted in the CG performance on the fixed surface. Only 4 out of 8 participants in the control group demonstrated a decreased ME post-intervention on the fixed surface. Whereas 6 out of 8 participants in the experimental group demonstrated a decrease in the ME post-intervention on the fixed surface. An average decrease of $25.3 \%$ was noted in ME of the experimental group during the $\mathrm{CG}$ performance on a fixed surface. Both the groups demonstrated a decreased ME on the compliant surface post-intervention. (see table 16) Only 5 out of 8 participants in the control group demonstrated a decrease in ME post-intervention as compared to all 8 participants in the experimental group demonstrating a post-intervention decrease in ME. 
Table 16: Results for Pre-Post changes of Movement Error (ME) for Target Game:

\begin{tabular}{|c|c|c|c|c|}
\hline CG task & \multicolumn{2}{|c|}{ CG_F } & \multicolumn{2}{c|}{ CG_C } \\
\hline OM & \multicolumn{2}{|c|}{ ME } & \multicolumn{2}{|c|}{ ME } \\
\hline & Pre & Post (Diff) & Pre & Post (Diff) \\
\hline C1 & 0 & $0.2(100 \%)$ & 0.5 & $0.3(-19.5 \%)$ \\
\hline C2 & 0 & $0.1(100 \%)$ & 0.4 & $0.01(-95 \%)$ \\
\hline C3 & 0.4 & $0.1(-78.7 \%)$ & 0.01 & $0.04(60 \%)$ \\
\hline C4 & 0.2 & $0.2(8.6 \%)$ & 0.5 & $0.1(-60.6 \%)$ \\
\hline C5 & 0.6 & $0.4(-24.3 \%)$ & 0 & $0.6(89.7 \%)$ \\
\hline C6 & 0.3 & $0.3(-4.6 \%)$ & 0.2 & $0(-100 \%)$ \\
\hline C7 & 0.3 & $0.6(34.1 \%)$ & 0.4 & $0.7(32.7 \%)$ \\
\hline C8 & 0.2 & $0.1(-39.4 \%)$ & 0.4 & $0.1(-75 \%)$ \\
\hline Avg. Diff & & $12 \%$ & & $-21 \%$ \\
\hline X1 & 0.1 & $0.1(11.1 \%)$ & 0 & $0(0 \%)$ \\
\hline X2 & 0.4 & $0.3(-13.2 \%)$ & 0.2 & $0.2(2.7 \%)$ \\
\hline X3 & 0.2 & $0(-84.6 \%)$ & 0.1 & $0.1(12.5 \%)$ \\
\hline X4 & 0.4 & $0.2(-43.4 \%)$ & 0.2 & $0.2(-4.5 \%)$ \\
\hline X5 & 0.6 & $0.3(-34.2 \%)$ & 0.03 & $0.02(-2 \%)$ \\
\hline X6 & 0.5 & $0.4(-10.8 \%)$ & 0.4 & $0.20(-34.4 \%)$ \\
\hline X7 & 0.3 & $0.4(1.5 \%)$ & 0.5 & $0.2(-48.6 \%)$ \\
\hline X8 & 0.2 & $0.1(-29 \%)$ & 0.3 & $0.2(-9.8 \%)$ \\
\hline Avg. Diff & & $-25.3 \%$ & & $-12.8 \%$ \\
\hline CBG V/s & & $37.5 \%$ & & -8.2 \\
\hline XG & & & & \\
\hline
\end{tabular}




\subsection{Qualitative Results:}

Tables 17 to 22 present the themes developed from the qualitative data from the semistructured interviews performed with the XG participant's parents in the qualitative section of this study. Six themes were identified. The first theme developed was Problem List (table 17) which demonstrates the problems of the children with CP stated by the parents of the participants in the experimental group. It was noted that most participants had the following issues (including but not limited to) frequent falls, imbalance, difficulty crossing obstacles, and lack of confidence in locomotion.

Table 17: Theme 1 - Problem List:

\begin{tabular}{|c|c|c|}
\hline Participant & Quotes from the Interview: & Problems/Categories \\
\hline $\mathrm{X} 1$ & $\begin{array}{l}\text { "He always had frequent falls while, crossing } \\
\text { hurdles/ obstacles." }\end{array}$ & Frequent Falls \\
\hline $\mathrm{X} 1$ & $\begin{array}{l}\text { "He always had frequent falls while crossing } \\
\text { hurdles/ obstacles." }\end{array}$ & Difficulty Crossing Huddles \\
\hline $\mathrm{X} 1$ & "He was not confident running." & Lack of confidence \\
\hline $\mathrm{X} 2$ & $\begin{array}{l}\text { "My daughter could not walk independently } \\
\text { initially," }\end{array}$ & Inability to walk independently \\
\hline $\mathrm{X} 2$ & "She had frequent falls." & Frequent Falls \\
\hline $\mathrm{X} 2$ & "She was scared to leave my hand \& walk" & Lack of confidence \\
\hline $\mathrm{X} 3$ & $\begin{array}{c}\text { "He always had his knees bent while standing } \\
\text { \& walking," }\end{array}$ & $\begin{array}{l}\text { Bent Knees (Knee Flexion } \\
\text { deformity) }\end{array}$ \\
\hline $\mathrm{X} 3$ & "had frequent falls." & Frequent Falls \\
\hline $\mathrm{X} 5$ & "my son did not walk independently;" & Inability to walk independently \\
\hline $\mathrm{X} 5$ & "he was scared to do so." & Lack of confidence \\
\hline X6 & $\begin{array}{l}\text { "My daughter while joining this therapy was } \\
\text { always scared to walk," }\end{array}$ & Lack of confidence \\
\hline
\end{tabular}


The second theme developed was Expectations (Table 18) which presents the expectations of the parents for their children affected with CP during the time of enrolment for this study. The common expectations at the time of enrolment were to improve their child's balance performance, improve their walking ability, enhance their concentration, and increase their confidence.

Table 18: Theme 2 - Expectations:

\begin{tabular}{|c|c|c|}
\hline Participant & Quotes from the Interview: & Expectations/Categories \\
\hline $\mathrm{X} 1$ & $\begin{array}{c}\text { "This therapy, which included both balancing as } \\
\text { well as playing the game simultaneously would } \\
\text { help him to become better at balance \& doing } \\
\text { challenging task" }\end{array}$ & Improve Balance \\
\hline $\mathrm{X} 2$ & $\begin{array}{c}\text { "This therapy where the balance was challenged } \\
\text { would help her improve her independence while } \\
\text { walking." }\end{array}$ & Independent Walking \\
\hline X3 & $\begin{array}{l}\text { "Standing on different surface would help him in } \\
\text { his balance. I thought this treatment would help } \\
\text { him with his balance issues." }\end{array}$ & Improve Balance \\
\hline $\mathrm{X} 4$ & $\begin{array}{c}\text { "Standing on different surfaces along with } \\
\text { playing games was the therapy \& I feel this } \\
\text { would him a lot, as it was very good to thing to } \\
\text { improve his balance" }\end{array}$ & Improve Balance \\
\hline $\mathrm{X} 4$ & "also his concentration." & Improve Concentration \\
\hline X5 & $\begin{array}{l}\text { "Before participating in this program, my son did } \\
\text { not walk independently; he was scared to do so. I } \\
\text { believed he would do so by playing the games } \\
\text { while standing on different platforms." }\end{array}$ & Independent walking \\
\hline $\mathrm{X} 6$ & $\begin{array}{l}\text { "I thought she would finally walk confidently, } \\
\text { thus enrolled her in the therapy. " }\end{array}$ & Confident Walking \\
\hline $\mathrm{X} 7$ & $\begin{array}{l}\text { "Playing the games while standing on different } \\
\text { surfaces would increase his concentration" }\end{array}$ & Improve concentration \\
\hline $\mathrm{X} 7$ & $\begin{array}{c}\text { "\& his balance. } \\
\text { I thought my son would finally concentrate on } \\
\text { one thing the games while standing/ balancing on } \\
\text { the floor, surfaces (bolster, balance disk)" }\end{array}$ & Improve Balance \\
\hline
\end{tabular}


The third theme developed was the Likes and Dislikes (Table 19) that presents the likes and dislikes of the participants about the CGR Balance intervention expressed through the views and opinions of their parents. It was observed through the responses that neither of the participants had any dislikes regarding the intervention. However, the parents did express the experiences of the challenges faced by their children due to the use of different surfaces and their difficulty levels to maintain balance while performing assigned tasks. The various difficulty levels of the games also challenged also the children during simultaneous balance and cognitive performance. Lastly, the parents expressed that they noticed their children adjusting to the activity progressions of the intervention after facing the initial discomfort of various balance and cognitive challenges. Nevertheless, most parents mentioned that participants loved the visual stimuli and their interaction with animated characters/colorful games throughout the duration of the intervention. 
Table 19: Theme 3 - Likes and Dislikes:

\begin{tabular}{|c|c|c|}
\hline Participant & Quotes from the Interview: & Choices/Categories \\
\hline $\mathrm{X} 1$ & "There was nothing I did not like." & No dislikes \\
\hline $\mathrm{X} 2$ & "I liked each and everything about the therapy." & Liked everything \\
\hline $\mathrm{X} 2$ & "Different surfaces was challenging for her." & Challenges- different surfaces \\
\hline $\mathrm{X} 2$ & $\begin{array}{l}\text { "Usually she loves colorful things, so these games } \\
\text { she enjoyed." }\end{array}$ & $\begin{array}{l}\text { Loved interacting with visual } \\
\text { stimuli }\end{array}$ \\
\hline $\mathrm{X} 3$ & $\begin{array}{l}\text { "as games got difficult, he was a little } \\
\text { uncomfortable initially" }\end{array}$ & Initial discomfort \\
\hline $\mathrm{X} 3$ & "but later he used to cope up \& play them" & Gradual Coping up \\
\hline $\mathrm{X} 3$ & $\begin{array}{l}\text { "The games got challenging as well, which he } \\
\text { really enjoyed." }\end{array}$ & Enjoyed Challenges \\
\hline $\mathrm{X} 4$ & $\begin{array}{l}\text { "When the games got difficult, it was challenging } \\
\text { for him to balance," }\end{array}$ & $\begin{array}{l}\text { Challenges- difficulty level of } \\
\text { games resulted in difficulty } \\
\text { balancing }\end{array}$ \\
\hline $\mathrm{X} 4$ & $\begin{array}{c}\text { "but later with practice, he was comfortable doing } \\
\text { it" }\end{array}$ & $\begin{array}{l}\text { Gradual coping up with } \\
\text { practice }\end{array}$ \\
\hline $\mathrm{X} 5$ & $\begin{array}{l}\text { "He loved all the games enjoyed watching the } \\
\text { cartoons \& playing the colorful games." }\end{array}$ & $\begin{array}{c}\text { Loved interacting with visual } \\
\text { stimuli }\end{array}$ \\
\hline $\mathrm{X} 6$ & $\begin{array}{l}\text { "She loved all the games. } \\
\text { Loved colorful cartoons \& thus was interested \& } \\
\text { played mindfully." }\end{array}$ & $\begin{array}{l}\text { Loved interacting with visual } \\
\text { stimuli }\end{array}$ \\
\hline $\mathrm{X} 7$ & "He loved colorful cartoons on the screen." & $\begin{array}{c}\text { Loved interacting with visual } \\
\text { stimuli }\end{array}$ \\
\hline
\end{tabular}


The fourth theme developed was the choices of the games (Table 20). This theme summarizes the most common choices of games for all the participants receiving the CGR program as expressed by their parents. The most favorite games of most participants included Zhu Zhu Pets, Bird's Town, Feeding Frenzy, and Chicken Invaders. Some parents also mentioned that their children loved all the games that were used for the CGR balance training protocol.

\section{Table 20: Theme 4 - Choices of games:}

\begin{tabular}{|c|c|c|}
\hline Participants & Quotes from the Interview: & Choice of games \\
\hline X1 & "He loved Zhu Zhu Pets \& Feeding frenzy" & $\begin{array}{c}\text { Zhu Zhu Pets } \\
\text { Feeding Frenzy }\end{array}$ \\
\hline X2 & "She loved all the games, chicken invaders she \\
liked the most." & Chicken Invaders \\
\hline X3 & "Aditya loved playing feeding frenzy, birds \\
town, zhu zhu pets" & $\begin{array}{c}\text { Feeding Frenzy } \\
\text { Bird's Town } \\
\text { Zhu Zhu Pets }\end{array}$ \\
\hline X4 & "Zhu zhu pets, feeding frenzy he enjoyed." & $\begin{array}{c}\text { Zhu Zhu Pets } \\
\text { Feeding Frenzy }\end{array}$ \\
\hline X5 & "Feeding frenzy, birds' town he enjoyed." & $\begin{array}{c}\text { Feeding Frenzy } \\
\text { Bird's Town }\end{array}$ \\
\hline X6 & "She loved all the games." & All games \\
\hline X7 & "The concentrated on the screen, loved \\
& playing chicken invaders \& birds town." & Bird's Town \\
\hline
\end{tabular}


The fifth theme developed was the Positive Effects of the Experimental Intervention (Table 21). This theme presents the commonly noted positive effects of the experimental intervention for the participants in the experimental group as opined by the parents of the participants in the CGR balance-training group. The effects such as improvement in balance performance, enhanced concentration, increased confidence, improved walking abilities, improvement in stair climbing, ease of obstacle crossing, increased lower limb control, improved knee extension, and better functional activity progression.

Table 21: Theme 5 - Positive Effects of Experimental Intervention:

\begin{tabular}{|c|c|c|}
\hline Participant & Quotes from the Interview: & Effects/Categories \\
\hline $\mathrm{X} 1$ & $\begin{array}{l}\text { "Different surfaces on which he stood improved } \\
\text { his balance I feel" }\end{array}$ & Improved Balance \\
\hline $\mathrm{X} 1$ & "His concentration improved." & Improved Concentration \\
\hline $\mathrm{X} 1$ & $\begin{array}{l}\text { "Yes he now crosses hurdles, climbs stairs } \\
\text { without losing his balance." }\end{array}$ & Improved Obstacle crossing \\
\hline $\mathrm{X} 1$ & $\begin{array}{l}\text { "Yes he now crosses hurdles, climbs stairs } \\
\text { without losing his balance." }\end{array}$ & Improved Stair climbing \\
\hline $\mathrm{X} 2$ & "She now is less fearful to walk." & Improved confidence \\
\hline $\mathrm{X} 2$ & $\begin{array}{l}\text { "Her activities like climbing on the bed / chair } \\
\text { has begun." }\end{array}$ & Activity Progression \\
\hline $\mathrm{X} 3$ & "His concentration also improved." & Improved Concentration \\
\hline $\mathrm{X} 3$ & $\begin{array}{l}\text { "Yes his knees are now more straight, he is } \\
\text { confident while walking, stair climbing, crossing } \\
\text { hurdles." }\end{array}$ & Improved Knee extension \\
\hline $\mathrm{X} 3$ & $\begin{array}{c}\text { "Yes his knees are now more straight, he is } \\
\text { confident while walking, stair climbing, crossing } \\
\text { hurdles." }\end{array}$ & Improved Confidence \\
\hline $\mathrm{X} 3$ & $\begin{array}{c}\text { "Yes his knees are now more straight, he is } \\
\text { confident while walking, stair climbing, crossing } \\
\text { hurdles." }\end{array}$ & Improved Stair Climbing \\
\hline $\mathrm{X} 3$ & $\begin{array}{c}\text { "Yes his knees are now more straight, he is } \\
\text { confident while walking, stair climbing, crossing } \\
\text { hurdles." }\end{array}$ & Improved Obstacle crossing \\
\hline $\mathrm{X} 4$ & $\begin{array}{c}\text { "Yes his balance has surely improved \& is more } \\
\text { confident." }\end{array}$ & Improved Balance \\
\hline
\end{tabular}




\begin{tabular}{|c|c|c|}
\hline X4 & $\begin{array}{c}\text { "Yes his balance has surely improved \& is more } \\
\text { confident." }\end{array}$ & Improved Confidence \\
\hline X5 & $\begin{array}{c}\text { "Yes my son now walks independently after the } \\
12 \text { weeks session." }\end{array}$ & Independent walking \\
\hline X6 & $\begin{array}{c}\text { "As she moves her head while playing the games, } \\
\text { her balance was challenged. This helped her } \\
\text { improve her control over her balance \& feet." }\end{array}$ & Improved Balance \\
\hline X6 & $\begin{array}{c}\text { "As she moves her head while playing the games, } \\
\text { her balance was challenged. This helped her } \\
\text { improve her control over her balance \& feet." }\end{array}$ & $\begin{array}{c}\text { Improved Lower Limb } \\
\text { Control }\end{array}$ \\
\hline X7 & "His concentration has increased" & Improved Concentration \\
\hline X7 & "Now stands \& walks with minimal falls." & Improved Standing and \\
walking
\end{tabular}


The final theme developed was the Future Expectations (Table 22). This theme records the responses to the question asked about their wish to continue the CGR Balance Training Program. As per the review of all the responses received from the parents of all participants in the CGR balance training group, all of them invariably expressed their interest in continuing this therapy if they were provided with the platform.

Table 22: Theme 6 - Future Expectation:

\begin{tabular}{|c|c|c|}
\hline Participant & Quotes from the Interview: & Expectation/Categories \\
\hline X1 & "Yes I would like to continue the therapy." & Continue Therapy \\
\hline X2 & $\begin{array}{c}\text { "Yes, I would love to continue this therapy } \\
\text { as it has worked well for my daughter." }\end{array}$ & Continue Therapy \\
\hline X3 & "Yes" & Continue Therapy \\
\hline X4 & "Yes." & Continue Therapy \\
\hline X5 & "Yes" & Continue Therapy \\
\hline X6 & "Yes" & Continue Therapy \\
\hline X7 & "Yes, I would love to continue the therapy." & \\
\hline
\end{tabular}




\section{Chapter 9: Discussion:}

\subsection{Known-Group Validity:}

The present study examined the magnitude of COP excursion during standing balance activities under single and dual-task conditions (VM tracking and Cognitive target game) for children with cerebral palsy (CP) and compared that with the typically developing (TD) children. No significant differences between the two groups were observed in the total path length of COP displacement during all tasks on the fixed surface or in the eyes open and cognitive game tasks on the compliant surface. Unexpectedly, the magnitude of COP excursion for the CP group was significantly less than the TD group for eyes closed and VM tracking tasks on the compliant surface. However, during the dual-task conditions, the performance of VM tracking and cognitive game tasks were significantly reduced in the $\mathrm{CP}$ group compared to the TD group on both surfaces.

These findings of the fixed surface tasks are consistent with the findings of the study reported by Donker et. al. (2008) They compared the magnitude of COP displacement during the performance of eyes open, eyes closed, and visual COP feedback tasks on the fixed surface between 10 TD children with 10 children affected with CP. The study reported no significant differences between the TD and CP groups during the standing balance tasks on the fixed surface. (Donker et al., 2008) It is also reported in the past that the EO condition on the firm surface does not differ widely between the TD children and ambulant CP children. (Dewar et. al.) Standing still for 30 seconds with eyes open on a firm/fixed surface can be done well (i.e. similar to TD) by children affected with $\mathrm{CP}$ and there is a high ceiling effect for this task.

In the present study, we observed that the children in the $\mathrm{CP}$ group demonstrated low body sway during the eyes closed task on the compliant surface as compared to the TD group. 
Standing on a compliant surface with vision eliminated is a challenging task. Typically, this task causes a significant increase in body sway and an increased threat to balance. (Desai et. al., 2010) It is possible that due to this increased threat to stability, the children with CP produced a stiffening strategy to minimize the amount of body and COP sway to prevent any episodes of loss of balance (LOB). This finding is consistent with the reports presented in the past that the $\mathrm{CP}$ children demonstrated overly constrained COP movement while the performance of difficult tasks such as EC task on the compliant surface. (Dewar et. al., 2021) The study reported no significant differences between the TD children and children affected with CP for the EC task on a compliant surface.

The present study also demonstrated that the $\mathrm{CP}$ group did not have any difference in the COP excursion between the EO and EC tasks on the compliant surface. Whereas, the TD group demonstrated a significantly higher COP excursion for the EC task as compared to the EO task, where there was significantly increased COP excursion with the elimination of vision. These findings also indicate that the $\mathrm{CP}$ group guarded themselves in response to the threat posed to their stability.

The children with CP demonstrated low COP sway during the VM tracking task on the sponge surface as compared to the TD children. However, it should be noted that many children with CP did not perform the tracking task. All the TD group participants (100\%) were engaged during the VM tracking task i.e. they were dual-tasking. On the other hand, more than $50 \%$ of participants in the $\mathrm{CP}$ group were not performing VM tracking tasks. It is likely that the reduced $\mathrm{COP}$ excursion of the $\mathrm{CP}$ group compared to the $\mathrm{TD}$ group occurred because the $\mathrm{CP}$ group participants were not dual-tasking. This finding also consistent with the absence of any significant difference in the TPL of children with CP for sponge EO and sponge VM tracking 
tasks. Whereas for the TD group, there was a significant increase in TPL during the sponge VM tracking dual-task condition as compared to the sponge EO single-task condition.

Although we did not include clinical cognitive assessment tools in our present study, we do have information on the visual-cognitive processing ability of the $\mathrm{CP}$ and TD group participants from the results of the cognitive game performance tasks performed on the fixed surface. These game tasks are considered to be single-task conditions for cognitive performance. The TD group performed better than the $\mathrm{CP}$ group in $\mathrm{CG}$ tasks when tested on the fixed surface i.e. single-task conditions. Thus, if the CP group performance is lower in absence of any physical demands, then this indicates the presence of some cognitive impairment.

\subsection{Feasibility of CGR platform for Balance Rehabilitation of children with CP:}

The present study project also incorporated a pilot intervention study to investigate the feasibility and benefits of the CGR platform use for balance rehabilitation in young children with CP. No adverse events were reported throughout the study period. The therapy compliance of both groups was reported to be $100 \%$.

The Minimal Clinically Important Difference (MCID) reported for standing subtest of GMFM for participants in GMFCS Level 1 is 2.4 units for GMFCS Level 2 is 3.3 units, for GMFCS Level 3 is 1.5 units, and overall MCID is 1.2 units. (Oeffinger et. al., 2008) The present study found a greater improvement pre to post-intervention of an increase of 5.4 units for the standing sub-test of GMFM for the XG group. Whereas for the CBG, the improvement in the standing sub-test of GMFM was observed to be an average increase of 2.1 units. The MCID reported for the walking, running, jumping subtest of GMFM is 4 units for GMFCS Level 1, 2.8 units for GMFCS Level 2, 1.8 units for GMFCS Level 3, and the overall MCID is reported to be 
1.6 units. (Oeffinger et. al., 2010) The present study found a greater improvement pre to postintervention of an increase of 5.0 units for the walking, running, jumping sub-test of GMFM for the $\mathrm{XG}$ group. Whereas for the $\mathrm{CBG}$, the improvement in the walking, running, jumping sub-test of GMFM was observed to be 1.1 units.

A Minimal Detectable Change (MDC) for PDMS-2 scores for the Gross Motor Quotient (including Stationary, Locomotion, and Reflexes subtests) is reported to be 4.98 units for children with CP. (Wang et al., 2006) The present study did not assess the Reflexes sub-test of the PDMS-2 as it was irrelevant for the current study context. The present study recorded an average increase of 6.3 units (including stationary and locomotion subtests) in the XG, which was better as compared to an average increase of 5.1 units (including stationary and locomotion subtests) in the CBG.

The MCID range for the PBS-total is reported to be 1.8 to 3.7 units for children with CP. (Chen et al., 2013) The present study found a greater improvement in the XG for PBS total scores that was an average increase of 5.38 units. Whereas, only 0.38 units of increase was observed in the CBG for the PBS.

The pre-to-post changes for all the clinical outcome measures observed in the present study are greater than the reported MCID values for both groups except for the post-intervention changes for the PBS scores for the CBG. The XG when compared to the CBG, demonstrated a greater improvement in all the gross motor functions and balance performance.

A study conducted by El-gohary et al. (2017) for use of the Biodex system for balance training in $\mathrm{CP}$ evaluated the balance and gross motor function of 48 children with spastic diplegic $\mathrm{CP}$ in the age range of 5-8 years. The control group received traditional PT including quadruped positioning, kneeling, and standing, along with balance exercises like standing on one 
leg, weight shifting, etc. on balance boards, mats, balls, etc. for a duration of 60 minutes thrice a week for 3 months. Whereas the experimental group received the traditional PT along with Biodex Balance Training. They started with a fixed surface and then progressed to different levels of platform movement in multiple directions, the participants were instructed to stand without holding on to the handrails and focus on the visual feedback provided through the Biodex platform (COP trajectory). They reported an increase of 2.2 units in the PBS score in the control group and 4.1 units in the experimental group of their study. The XG in the present study demonstrated a greater pre to post-intervention improvement of 9.8\% in the PBS scores whereas the experimental group of the Biodex study demonstrated a $7.4 \%$ improvement in postintervention. (El-gohary et al., 2017)

A study conducted by El Shamy \& Abd El Kafy (2014) used the Biodex system based on COM signal for balance assessment and rehabilitation in 30 children with $\mathrm{CP}$ randomized to control and study groups. The control group received conventional PT including stretching, strengthening, standing, balance exercises, and gait training for 2 hours. The experimental group received a combination of conventional PT (90 minutes) and Biodex balance training (30 minutes). During training, children were instructed to move the computer cursor by shifting their COM to interact with the visual targets appearing on the screen (such as a flashing ball). This intervention was continued for 3 months at a frequency of 3 sessions per week. They reported a $1.2 \%$ decrease in body sway during the EO standing balance performance in the control group and $2.6 \%$ in the experimental group. The present study observed a greater decline for the COP excursion in the XG that was a $28.8 \%$ post-intervention decrease in the TPL for the EO task on the fixed surface. Whereas only a $2.4 \%$ post-intervention decrease in the TPL for the CBG was observed. (Table 12) The XG performed $26.4 \%$ better in the EO task on the fixed surface and 
$15.2 \%$ better in the EC task on the fixed surface as compared to the CBG. The present study also assessed the participants' balance performance while dual-tasking on the fixed surface. The XG demonstrated a $36 \%$ and $37 \%$ decrease in the TPL while the performance of VM tracking and cognitive gaming tasks on the fixed surface respectively. Whereas the CBG demonstrated a $21 \%$ and $11 \%$ decrease in the TPL while the performance of the VM tracking and cognitive gaming tasks on the fixed surface. The studies in the literature have used the fixed surface for balance assessment and training, which does not challenge the balance for most ambulatory children with CP. (Dewar et al., 2021)

The present study also observed the post-intervention changes for both groups during the performance of mCTSIB tasks and dual-tasks on the compliant surface. The XG demonstrated a better improvement in all tasks on the compliant surface. The decrease in TPL was found to be $13 \%$ for the EO task, $5 \%$ for the EC task, $9 \%$ for the VM tracking task, and $18 \%$ for the cognitive gaming task on the compliant surface. On the other hand, the CBG demonstrated an increase in TPL post-intervention in 3 of the 4 assessment tasks on the compliant surface. The TPL was observed to be increased by $8.5 \%$ for the EO task, by $6.8 \%$ for the EC task, by $8.1 \%$ for the VM tracking task, and a decrease of $14 \%$ was observed for the cognitive gaming task on the compliant surface. Unfortunately, no study in the literature reported the standing balance assessments on complaint surface or while the performance of dual-tasks as this study does; thus, no comparisons could be made for the COP assessment for these aspects of advanced balance skills.

These findings of the quantitative results for the $\mathrm{XG}$ are also consistent with the qualitative findings of the present study as expressed by the parents of the XG participants. (Table 21) The parents of all XG participants mentioned an improved balance after the 
completion of 12 weeks of CGR balance training. (Table 21) Some parents mentioned that their child had decreased episodes of falls and better standing and locomotion post-intervention (Table 21) The XG participants' parents recognized that their expectations at the time of enrolment to this study related to balance performance, locomotion capabilities, and activity progression from standing to walking were addressed during the 12-week CGR balance-training program. (Table $18,19,21)$ As per the literature, participant's concentration and adherence to therapy determine the extent of the therapy effect that transfers to the functional activities. (Taub et al., 2004) Parents acknowledged that their children presented an increased level of concentration and motivation to perform their exercises. (Table 21) They also mentioned that their children demonstrated better independence in their gross functions of daily living like walking, obstacle crossing, etc. after the 12-weeks of CGR balance rehabilitation therapy. (Table 21)

It is reported in the past that long-term therapy regimens with intensive, repetitive, taskoriented practice-based exercises often fail to remain engaging for young children with CP. Low compliance to such therapy regimens is often reported, as it is very difficult to maintain a high level of motivation among these children and keep them engaged in challenging repetitive exercises. (Reid 2002) Thus, the CGR platform was developed with these shortcomings in mind. (Szturm et al., 2014, Bhatt, 2018, Szturm et al., 2017, and Nankar et al., 2017) The component of engagement and motivation was taken into consideration while planning the CGR based balance rehabilitation program along with keeping the tasks challenging at all times for improvement in balance and cognition. Thus, the DT assessments included a cognitive assessment to evaluate the visual-motor tracking and cognitive processing abilities of the study participants while the performance of challenging balance tasks. 
The visuomotor tracking performance results demonstrated a decline in the average residual error in the $\mathrm{XG}$ post-intervention. (Table 14) The XG demonstrated a $3.6 \%$ decline in the ARE on the compliant surface and the CBG had no change post-intervention. In fact, some participants in the $\mathrm{CBG}$ demonstrated an increase in ARE post-intervention during the performance of VM tracking task on the compliant surface. (Table 14) The cognitive game performance demonstrated a similar result for both groups. (Table 15, 16) The XG demonstrated a decline of $25 \%$ in the movement error post-intervention on the fixed surface whereas the CBG demonstrated an increase of $12 \%$ post-intervention. (Table 16) This likely is due to the fact that the therapy program for the CBG did not include cognitive loading with game tasks or similar cognitive activities.

The XG participants were performing balance activities along with multiple game events at a varying level of difficulty (i.e. dual-tasking) during their therapy sessions. Participants' head rotation movements used to interact with the game would challenge their balance by increasing the amount of body sway and especially when standing on a compliant surface. The performance of game tasks using head rotation movements while maintaining balance to prevent LOB is an additional level of difficulty. The parents' of the XG participants also expressed the challenges faced by their children due to the various levels of difficulty caused by the use of increased balance cost through the means of a variety of compliant surfaces like air bladder, bolster, therapy ball, etc., as well as increasing difficulty levels of games. (Table 19) The increased game difficulty with the speed of movement, optokinetic stimuli, increased visual distractors, etc. added another level of challenge for the participants for maintaining their concentration on game performance, use head rotation for gameplay, and simultaneously use strategies to prevent any episodes of LOB/falls. However, parents noted that regular practice of these difficult tasks made 
it comfortable for their children to cope with the different levels of activity progressions. (Table $19,21)$ There was a better performance improvement noted in the XG in all the functional domains as they were engaged in therapy and were challenged at all times for motor as well as cognitive processing functions. On the other hand, the CBG did not demonstrate a huge effect of therapy as it is also reported in the past that the repetition of intensive exercises is considered tedious and difficult to accomplish due to the same uninteresting exercises. Studies in the past comparing the use of digital media in rehabilitation with traditional physiotherapy have reported similar findings. (Peungsuwan et al., 2017 and Wu et al., 2019)

The CGR program was made engaging and fun by the addition of interactive commercial games of varying difficulty levels. This component of cognitive gaming added a cognitive load to therapy, likely causing Dual-Task Interference (DTI) for the standing balance performance. A study conducted by Elhindi et al., (2016) evaluating the effects of DT training on balance performance of children with infantile hemiparesis also reported that dual-task training leads to cortical plasticity resulting in improved motor plasticity thus improving the functional outcome of therapy. Many children with $\mathrm{CP}$ require greater cognitive effort to accomplish challenging functional tasks. Thus, when these participants are challenged with the simultaneous performance of balance and cognitive tasks, it affects their task performance. Eventually, this may result in a state of competition in the use of the available cognitive resources between the two tasks. Consequently, these factors result in DTI i.e. disturbed balance or cognitive performance or both causing an increased challenge for DT performance. (Roostaei et.al., 2021) Dual-tasking of specific DT Balance Training while performing visuomotor tracking and cognitive computer game tasks requires visual search and tracking, information processing, and organization of multiple sensory systems actions due to the determined state of balance. During 
the performance of visual-spatial processing tasks of VM tracking and CG while maintaining balance on the compliant surface, the participants would be required to use the existing attentional resources for processing the visual-spatial information. For instance, the performance of the cognitive tasks requires executive cognitive functions of visual attention, visual searching, cognitive inhibition, spatial processing of the randomly moving targets, and motor planning of the head rotations to move the game paddle. The balance activities also require executive cognitive functions in particular processing of spatial information from visual, vestibular, and somatosensory systems to determine the spatial orientation and the state of body balance. This would result in increased demand on processing in the parietal cortex and the prefrontal cortex because of the interference between the motor and cognitive demands, i.e., DTI. (Li et. al., 2018 and Yu et. al., 2021) This divided attention and processing overlap of spatial processing requirements between the cognitive game tasks and corrective balance responses to prevent loss of balance likely would result in dual-task interference of one or both of the motor and cognitive tasks. The participant is required to maintain their balance within the base of support (fixed or sponge surface) and generate timely corrective responses to prevent any episodes of falls. Along with this motor control, participants are simultaneously required to overlap (in the VM tracking task) or achieve the target (in the CG task) by continuous foveation. There is repeated use of the anticipatory or feedforward balance control for maintenance of balance while performing the dual-task balance training. As the dual-task balance training on the complaint surface involves more sudden or unpredictable balance costs as compared to conventional balance training, the participants require more reactive balance control strategies while performing CGR DT balance training. The neural circuits are burdened, as this executive cognitive processing is required 
simultaneously with motor control, to organize, coordinate, and execute the smooth performance of balance tasks along with cognitive gaming i.e., DT.

As observed in the known-group validity outcomes of the present study, the CP children had reduced balance and cognitive performance during the DT condition as compared to the TD children. Thus, when the CP participants were specifically trained for balance with respective protocols, an improvement was noted in the balance performance of both groups. As the XG group was also involved in intensive DT i.e., balancing along with the performance of cognitive gaming, there was an improved performance noted on the visual tracking and cognitive gaming post-intervention.

In the present study, the assessment tasks were standardized for both groups with the use of the same compliant surface and cognitive loading for all participants. This provided standardized repeatable balance, computer tracking, and cognitive game activities on both test days, and for all test conditions (fixed and complaint surface). Imperious performance levels were observed in the present study for both VM tracking and CG tasks during the complaint surface balance task in the XG along with a better improvement in the reactive balance control. This likely indicates that the participants were attending to and processing the information they saw on the display. This is because the compliant surface dual-task balance training involves reactive balance and dynamic stability training along with a good amount of cognitive loading. However, the CBG demonstrated modest performance levels for reactive balance responses and CG tasks. This demonstrates that conventional balance training lacks the focus on cognitive loading and dual-task training.

The parents' of the XG participants recognized that the use of difficult complaint surfaces for balance training along with dual-task training with the addition of fun, interactive computer 
gaming increased the challenges posed in front of their children. (Table 19) The XG demonstrated a larger pre-to-post change as compared to the $\mathrm{CBG}$, as the $\mathrm{XG}$ participants were found to be engaged and motivated throughout the study duration by the means of the therapeutic games as expressed by their parents during the interviews. This benefit of gaming and digital media is also reported in the past. (Wu et al., 2019, Miller et.al, 2004, Kelders et al., 2018, and Sober \& Sabes, 2003) As per parent's views, the ability to experience the performance of game tasks with colorful backgrounds and attractive characters enhanced their children's concentration and motivation to perform the intensive balance gaming tasks repeatedly. (Table 19) The XG protocol was designed to have changes in games with increasing difficulty levels i.e. challenging dual-task training as tolerated by each participant. Whereas, the CBG had limited opportunities for changes or novelties in their protocol.

Upon enquiring about the desire to continue the CGR balance training program for the XG after the study duration, the XG participants' parents invariably expressed their interest in continuing the protocol if provided with the platform in the right setting. Similar interest for the continued use of this platform for balance and gait training in a population with Parkinson's Disease was reported by Mahana et. al. in 2019. (Mahana et. al., 2019) The study conducted by Kanitkar et. al., using CGR for hand function rehabilitation also reported the desire of the parents to have this platform available for therapeutic use for their children affected with CP. (Kanitkar et. al. 2021) 


\section{Chapter 10: Limitations:}

There are few limitations of this study. A limitation noted at the stage of recruitment was a restricted inclusion criterion limited to children affected with $\mathrm{CP}$. In addition, as the inclusion criteria did not allow the inclusion of children affected with CP in GMFCS levels 4 and 5, the results of this study cannot be generalized to the entire $\mathrm{CP}$ population.

The use of a screening tool to address the cognitive impairment levels at the beginning of the study to choose the appropriate games and levels of difficulty for visuomotor interaction would be beneficial to plan the treatment sessions in a better capacity and evaluate the cognitive changes.

A few drawbacks in the assessment protocol were also noticed after a close evaluation of the study data. The difficulty level for the balance task performance (surface) and cognitive task performance (difficult game task) was not challenging enough to create more distinction between the easy and difficult tasks. The difficult cognitive game task was not difficult enough as just a change of the trajectory of the object from straight to diagonal did not create a great impact of the task difficulty on the game performance.

The qualitative section of this study lacked the involvement of the parents of the children in the CBG. No comparisons of the experiences of the XG parents was possible to compare the impact of CGR on the engagement and motivation levels of the participants. The use of a participation level assessment tool was not incorporated in the study, which could have justified the observation and expressions of the participant's parents.

The present study did not assess the carry over effect of the CGR Balance training as there was no follow-up assessment in the present study. 


\section{Chapter 11: Future Steps:}

\section{1: Recommendations for Full-Scale RCT:}

The addition of a screening tool for neuropsych testing and participation level assessment tool in children affected with $\mathrm{CP}$ or $\mathrm{ABI}$ would help examine the baseline cognitive functions and participation levels of all the study participants. For instance, a Mini-Mental Scale Evaluation for children (MMSE) (Jain \& Passi, 2005) for cognitive screening before recruitment for intervention study. The Canadian Occupation Performance Measure (COPM) (Kang et. al., 2020) could be used as an outcome measure for the assessment of participation levels of the study participants before and after respective interventions.

The CGR assessment protocol could involve the use of a more challenging compliant surface like different sponge surface or a balance disk to increase the level of difficulty/challenge between the two CGR balance assessment tasks. Besides, the difficulty level of the CG2 could be increased to have more distinction between the two game tasks (i.e. CG1 and CG2). For instance, addition of an optokinetic background for visual processing difficulty or an increased speed of the target objects could result in a more challenging cognitive assessment game task.

It is important to develop evidence on the actual dosage of an intervention to be delivered. Most of the studies in the literature do not provide the amplitude, speed, and frequency of movements performed during therapy. This makes it difficult to understand the relationship between the actual volume of practice and the outcomes of therapy. Thus, the dosage of balance exercise should be determined by a full-scale RCT. This can be done by recording the actual amount of time spent on playing different types of games while performing balance tasks, the 
difficulty level at which the games were played, and the average amount of displacement of COP while performing DT Balance training. This information can be used to quantify the intensity and duration of each exercise (i.e. therapy dosage).

As per the MCID \& MDC studies for GMFM-88 (Oeffinger et. al., 2010), PDMS-2

(Wang et al., 2006), and PBS (Chen et. al., 2013), the effects were evaluated after 6-18 months. To incorporate that intensity and duration of treatment, a follow-up assessment to investigate the carry-over effect of the interventions would be helpful. A long-term intervention/ follow-up assessment after 6-18 months could be incorporated to look at the sustained treatment effects as seen in the MCID studies of the clinical outcome measures. (Oeffinger et. al., 2010, Wang et al., 2006, and Chen et. al., 2013)

\section{2: Future Study Considerations:}

This research project is a paradigm shift that will lead to efficient and pragmatic interventions and will transfer new knowledge into clinical practice that will advance treatments of chronic neurodevelopmental disorders for young children. The near future goals are to proceed with a full-scale RCT with all the proposed changes in the protocol of assessment. The next step would be to evaluate the effectiveness of the CGR platform for locomotor training in young children with neurodevelopmental disorders. A gradual introduction of this platform at a wide range of community centers and physiotherapy rehabilitation centers will enhance the balance training regimens at various levels. This program will build capacity in the emerging area of rehabilitation using computer-aided and game-assisted strategies aimed at assessing and treating CP. Gradually this platform can be moved to home rehabilitation with proper safety training as it is proven to be user-friendly and affordable. 
This pilot study of balance training for children with moderate to severe CP demonstrates a potential to be a successful logistically, cost-efficient, and effective form of treatment producing positive neuro-motor and functional outcomes. As a result, serious consideration should be given to its further progression to a full-scale RCT. This pilot study was successful in creating the protocol of treatment and assessment/performance monitoring that could be applied to a randomized control trial of the efficacy of this intervention. The most important principle noted was that careful planning is required to provide a sufficient amount of difficulties in balance and dual-task training while simultaneously giving participants multiple opportunities to engage while challenging themselves through being out of balance, with and without dual task demands. 


\section{References}

Rosenbaum, P., Paneth, N., Leviton, A., Goldstein, M., Bax, M., Damiano, D., Dan, B., \& Jacobsson, B. (2007). A report: the definition and classification of cerebral palsy April 2006. Developmental Medicine and Child Neurology, 49(109), 8-14.

https://doi.org/10.1111/j.1469-8749.2007.tb12610.x

Stavsky, M., Mor, O., Mastrolia, S. A., Greenbaum, S., Than, N. G., \& Erez, O. (2017).

Cerebral palsy-trends in epidemiology and recent development in prenatal mechanisms of disease, treatment, and prevention. Frontiers in Pediatrics, 5, 21-21. https://doi.org/10.3389/fped.2017.00021

Robertson, C. M. ., Ricci, M. F., O’Grady, K., Oskoui, M., Goez, H., Yager, J. Y., \& Andersen, J. C. (2017). Prevalence Estimate of Cerebral Palsy in Northern Alberta: Births, 2008-2010. Canadian Journal of Neurological Sciences, 44(4), 366-374.

https://doi.org/10.1017/cjn.2017.33

Van Naarden BK, Doernberg N, Schieve L, Christensen D, Goodman A, Yeargin-Allsopp M. Birth prevalence of cerebral palsy: a population-based study. Pediatrics.

2016;137. https://doi.org/10.1542/peds.2015-2872.

Gulati, S., \& Sondhi, V. (2018). Cerebral Palsy: An Overview. Indian Journal of Pediatrics, 85(11), 1006-1016. https://doi.org/10.1007/s12098-017-2475-1 
Peter R, Nigel P, Alan L, Murray G MB. A report: the definition and classification of cerebral palsy. Dev Med Child Neurol [Internet]. 2007;47: 510.(1):3-7. Available from: https://www.researchgate.net/publication/285476231\%0

Cao, J., Khan, B., Hervey, N., Tian, F., Delgado, M. R., Clegg, N. J., Smith, L., Roberts, H., Tulchin-Francis, K., Shierk, A., Shagman, L., MacFarlane, D., Liu, H., \& Alexandrakis, G. (2015). Evaluation of cortical plasticity in children with cerebral palsy undergoing constraintinduced movement therapy based on functional near-infrared spectroscopy. Journal of Biomedical Optics, 20(4), 046009-046009. https://doi.org/10.1117/1.JBO.20.4.046009

Wolf, S. L., Winstein, C. J., Miller, J. P., Taub, E., Uswatte, G., Morris, D., Giuliani, C., Light, K. E., Nichols-Larsen, D., \& EXCITE Investigators, for the. (2006). Effect of Constraint-Induced Movement Therapy on Upper Extremity Function 3 to 9 Months After Stroke: The EXCITE Randomized Clinical Trial. JAMA : the Journal of the American Medical Association, 296(17), 2095-2104. https://doi.org/10.1001/jama.296.17.2095

Liepert, J. (2006). Motor cortex excitability in stroke before and after constraint-induced movement therapy. Cognitive and Behavioral Neurology, 19(1), 41-47. https://doi.org/10.1097/00146965-200603000-00005 
Tarkka, I. M., Pitkänen, K., \& Sivenius, J. (2005). Paretic hand rehabilitation with constraintinduced movement therapy after stroke. American Journal of Physical Medicine \& Rehabilitation, 84(7), 501-505. https://doi.org/10.1097/01.phm.0000166881.71097.9d

Rijntjes, M., Hobbeling, V., Hamzei, F., Dohse, S., Ketels, G., Liepert, J., \& Weiller, C. (2005). Individual Factors in Constraint-Induced Movement Therapy after Stroke. Neurorehabilitation and Neural Repair, 19(3), 238-249. https://doi.org/10.1177/1545968305279205

Martin, A., Burtner, P. A., Poole, J., \& Phillips, J. (2008). Case report: ICF-level changes in a preschooler after constraint-induced movement therapy. The American Journal of Occupational Therapy, 62(3), 282-288. https://doi.org/10.5014/ajot.62.3.282

Taub, E., Ramey, S. L., DeLuca, S., \& Echols, K. (2004). Efficacy of Constraint-Induced Movement Therapy for Children With Cerebral Palsy With Asymmetric Motor Impairment. Pediatrics (Evanston), 113(2), 305-312. https://doi.org/10.1542/peds.113.2.305

Barreca, S., Wolf, S. L., Fasoli, S., \& Bohannon, R. (2003). Treatment Interventions for the Paretic Upper Limb of Stroke Survivors: A Critical Review. Neurorehabilitation and Neural Repair, 17(4), 220-226. https://doi.org/10.1177/0888439003259415 
Malhi, P., \& Singhi, P. (2002). Role of parents evaluation of developmental status in detecting developmental delay in young children. Indian pediatrics, 39(3), 271-275. http://www.ncbi.nlm.nih.gov/pubmed/11910137

Yang, J. F., Livingstone, D., Brunton, K., Kim, D., Lopetinsky, B., Roy, F., Zewdie, E., Patrick, S. K., Andersen, J., Kirton, A., Watt, J.-M., Yager, J., \& Gorassini, M. (2013). Training to Enhance Walking in Children With Cerebral Palsy: Are We Missing the Window of Opportunity? Seminars in Pediatric Neurology, 20(2), 106-115. https://doi.org/10.1016/j.spen.2013.06.011

Novak, I., Morgan, C., Adde, L., Blackman, J., Boyd, R. N., Brunstrom-Hernandez, J., Cioni, G., Damiano, D., Darrah, J., Eliasson, A.-C., de Vries, L. S., Einspieler, C., Fahey, M., Fehlings, D., Ferriero, D. M., Fetters, L., Fiori, S., Forssberg, H., Gordon, A. M., ... Badawi, N. (2017). Early, Accurate Diagnosis and Early Intervention in Cerebral Palsy: Advances in Diagnosis and Treatment. JAMA Pediatrics, 171(9), 897-907. https://doi.org/10.1001/jamapediatrics.2017.1689

Reid, L. B., Rose, S. E., \& Boyd, R. N. (2015). Rehabilitation and neuroplasticity in children with unilateral cerebral palsy. Nature Reviews. Neurology, 11(7), 390-400. https://doi.org/10.1038/nrneurol.2015.97

Wilkins, K. B., Owen, M., Ingo, C., Carmona, C., Dewald, J. P. ., \& Yao, J. (2017). Neural plasticity in moderate to severe chronic stroke following a device-assisted task-specific 
arm/hand intervention. Frontiers in Neurology, 8, 284-284.

https://doi.org/10.3389/fneur.2017.00284

Harvey, R. L. (2009). Improving poststroke recovery: Neuroplasticity and task-oriented training. Current Treatment Options in Cardiovascular Medicine, 11(3), 251-259. https://doi.org/10.1007/s11936-009-0026-4

Hubbard, I. J., Parsons, M. W., Neilson, C., \& Carey, L. M. (2009). Task-specific training: evidence for and translation to clinical practice. Occupational Therapy International, 16(3-4), 175-189. https://doi.org/10.1002/oti.275

Teasell, R. W., Foley, N. C., Salter, K. L., \& Jutai, J. W. (2008). A Blueprint for Transforming Stroke Rehabilitation Care in Canada: The Case for Change. Archives of Physical Medicine and Rehabilitation, 89(3), 575-578.

https://doi.org/10.1016/j.apmr.2007.08.164

Toovey, R., Bernie, C., Harvey, A., Mcginley, J., Spittle, A., (2017) Task-specific gross motor skills training for ambulant school aged children with cerebral palsy: a systematic review. Developmental Medicine and Child Neurology, 59, 108-109. https://doi.org/10.1111/dmcn.49_13512 
Willoughby, K. L., Dodd, K. J., \& Shields, N. (2009). A systematic review of the effectiveness of treadmill training for children with cerebral palsy. Disability and Rehabilitation, 31(24), 1971-1979. https://doi.org/10.3109/09638280902874204

Sullivan, K. J., Brown, D. A., Klassen, T., Mulroy, S., Ge, T., Azen, S. P., \& Winstein, C. J. (2007). Effects of Task-Specific Locomotor and Strength Training in Adults Who Were Ambulatory After Stroke: Results of the STEPS Randomized Clinical Trial. Physical Therapy, 87(12), 1580-1602. https://doi.org/10.2522/ptj.20060310

Maier, M., Ballester, B. R., \& Verschure, P. F. M. J. (2019). Principles of Neurorehabilitation After Stroke Based on Motor Learning and Brain Plasticity Mechanisms. Frontiers in Systems Neuroscience, 13, 74-74. https://doi.org/10.3389/fnsys.2019.00074

Boyd, L. A., Vidoni, E. D., \& Wessel, B. D. (2010). Motor learning after stroke: Is skill acquisition a prerequisite for contralesional neuroplastic change? Neuroscience Letters, 482(1), 21-25. https://doi.org/10.1016/j.neulet.2010.06.082

Salem, Y., \& Godwin, E. M. (2009). Effects of task-oriented training on mobility function in children with cerebral palsy. NeuroRehabilitation (Reading, Mass.), 24(4), 307-313. https://doi.org/10.3233/NRE-2009-0483

Kumban, W., Amatachaya, S., Emasithi, A., \& Siritaratiwat, W. (2013). Effects of taskspecific training on functional ability in children with mild to moderate cerebral palsy. 
Developmental Neurorehabilitation, 16(6), 410-417.

https://doi.org/10.3109/17518423.2013.772672

Mastos, M., Miller, K., Eliasson, A. C., \& Imms, C. (2007). Goal-directed training: linking theories of treatment to clinical practice for improved functional activities in daily life. Clinical Rehabilitation, 21(1), 47-55.https://doi.org/10.1177/0269215506073494

Wulf, G., \& Prinz, W. (2001). Directing attention to movement effects enhances learning: A review. Psychonomic Bulletin \& Review, 8(4), 648-660. https://doi.org/10.3758/BF03196201

Locke, E. A., \& Latham, G. P. (2002). Building a practically useful theory of goal setting and task motivation. A 35-year odyssey. The American Psychologist, 57(9), 705-717. https://doi.org/10.1037//0003-066X.57.9.705

Bandura, A., Barbaranelli, C., Caprara, G. V., \& Pastorelli, C. (2001). Self-Efficacy Beliefs as Shapers of Children's Aspirations and Career Trajectories. Child Development, 72(1), 187-206.https://doi.org/10.1111/1467-8624.00273

ØstensjØ, S., Øien, I., \& Fallang, B. (2008). Goal-oriented rehabilitation of preschoolers with cerebral palsy-a multi-case study of combined use of the Canadian Occupational Performance Measure (COPM) and the Goal Attainment Scaling (GAS). Developmental Neurorehabilitation, 11(4), 252-259. https://doi.org/10.1080/17518420802525500 
Bottos, M., Feliciangeli, A., Sciuto, L., Gericke, C., \& Vianello, A. (2001). Functional status of adults with cerebral palsy and implications for treatment of children. Developmental Medicine and Child Neurology, 43(8), 516-528. https://doi.org/10.1111/j.1469$\underline{8749.2001 . t b 00755 . x}$

Ketelaar, M., Vermeer, A., Hart, H., van Petegem-van Beek, E., \& Helders, P. J. (2001). Effects of a Functional Therapy Program on Motor Abilities of Children With Cerebral Palsy. Physical Therapy, 81(9), 1534-1545. https://doi.org/10.1093/ptj/81.9.1534

Trahan, J., \& Malouin, F. (2002). Intermittent intensive physiotherapy in children with cerebral palsy: a pilot study. Developmental Medicine and Child Neurology, 44(4), 233-239. https://doi.org/10.1017/S0012162201002006

Odman, P., \& Oberg, B. (2006). Effectiveness and expectations of intensive training: A comparison between child and youth rehabilitation and conductive education. Disability and Rehabilitation, 28(9), 561-570. https://doi.:?mnorg/10.1080/00222930500218821

Peungsuwan, P., Parasin, P., Siritaratiwat, W., Prasertnu, J., \& Yamauchi, J. (2017). Effects of Combined Exercise Training on Functional Performance in Children With Cerebral Palsy: A Randomized-Controlled Study. Pediatric Physical Therapy, 29(1), 39-46. https://doi.org/10.1097/PEP.0000000000000338 
Qi, Y.-C., Niu, X.-L., Gao, Y.-R., Wang, H., Hu, M., Dong, L.-P., \& Li, Y.-Z. (2018). Therapeutic Effect Evaluation of Neuromuscular Electrical Stimulation With or Without Strengthening Exercise on Spastic Cerebral Palsy. Clinical Pediatrics, 57(5), 580-583. https://doi.org/10.1177/0009922817732619

Surana, B. K., Ferre, C. L., Dew, A. P., Brandao, M., Gordon, A. M., \& Moreau, N. G. (2019). Effectiveness of Lower-Extremity Functional Training (LIFT) in Young Children With Unilateral Spastic Cerebral Palsy: A Randomized Controlled Trial. Neurorehabilitation and Neural Repair, 33(10), 862-872. https://doi.org/10.1177/1545968319868719

Nelson, M. E., Rejeski, W. J., Blair, S. N., Duncan, P. W., Judge, J. O., King, A. C., Macera, C. A., \& Castaneda-Sceppa, C. (2007). Physical activity and public health in older adults: Recommendation from the American College of Sports Medicine and the American Heart Association. Circulation (New York, N.Y.), 116(9), 1094-1105.

https://doi.org/10.1161/CIRCULATIONAHA.107.185650

Society, A. G., Society, G., Of, A. A., \& On Falls Prevention, O. S. P. (2001). Guideline for the Prevention of Falls in Older Persons. Journal of the American Geriatrics Society (JAGS), 49(5), 664-672.https://doi.org/10.1046/j.1532-5415.2001.49115.x

Hsu, S.-S., Hu, M.-H., Wang, Y.-H., Yip, P.-K., Chiu, J.-W., \& Hsieh, C.-L. (2010). Doseresponse relation between neuromuscular electrical stimulation and upper-extremity function 
in patients with stroke. Stroke (1970), 41(4), 821-824.

https://doi.org/10.1161/STROKEAHA.109.574160

Power, V., \& Clifford, A. M. (2013). Characteristics of optimum falls prevention exercise programmes for community-dwelling older adults using the FITT principle. European Review of Aging and Physical Activity, 10(2), 95-106. https://doi.org/10.1007/s11556-012$\underline{0108-2}$

Williams, D. M. (2008). Exercise, affect, and adherence: An integrated model and a case for self-paced exercise. Journal of Sport \& Exercise Psychology, 30(5), 471-496.

https://doi.org/10.1123/jsep.30.5.471

Wu, J., Loprinzi, P. D., \& Ren, Z. (2019). The Rehabilitative Effects of Virtual Reality Games on Balance Performance among Children with Cerebral Palsy: A Meta-Analysis of Randomized Controlled Trials. International Journal of Environmental Research and Public Health, 16(21), 4161-. https://doi.org/10.3390/ijerph16214161

Sober, S. J., \& Sabes, P. N. (2003). Multisensory Integration during Motor Planning. The Journal of Neuroscience, 23(18), 6982-6992. https://doi.org/10.1523/JNEUROSCI.23-18$\underline{06982.2003}$ 
Dewar, R., Love, S., \& Johnston, L. M. (2015). Exercise interventions improve postural control in children with cerebral palsy: a systematic review. Developmental Medicine and Child Neurology, 57(6), 504-520. https://doi.org/10.1111/dmcn.12660

Ledebt, A., Becher, J., \& Savelsbergh, G. J. . (2005). 14.13 Balance training with visual feedback in children with hemiplegic cerebral palsy. Gait \& Posture, 21, S86-S86. https://doi.org/10.1016/S0966-6362(05)80283-8

El-Shamy, S. M., \& Abd El Kafy, E. M. (2014). Effect of balance training on postural balance control and risk of fall in children with diplegic cerebral palsy. Disability and Rehabilitation, 36(14), 1176-1183. https://doi.org/10.3109/09638288.2013.833312

El-gohary, T. M., Emara, H. A., Al-Shenqiti, A., \& Hegazy, F. A. (2017). Biodex balance training versus conventional balance training for children with spastic diplegia. Journal of Taibah University Medical Sciences, 12(6), 534-540.

https://doi.org/10.1016/j.jtumed.2017.07.002

Abd El-Kafy, E. M., \& El-Basatiny, H. M. Y. M. (2014). Effect of Postural Balance Training on Gait Parameters in Children with Cerebral Palsy. American Journal of Physical Medicine \& Rehabilitation, 93(11), 938-947. https://doi.org/10.1097/PHM.0000000000000109

Kozyavkin, V. I., Kachmar, B. O., Terletskyy, O. I., Kachmar, O. O., \& Ablikova, I. V. (2013). Stepping games with Dance Mat for motor rehabilitation. 2013 International 
Conference on Virtual Rehabilitation (ICVR), 174-175.

https://doi.org/10.1109/ICVR.2013.6662108

Hsieh, H.-C. (2018). Effects of a Gaming Platform on Balance Training for Children With Cerebral Palsy. Pediatric Physical Therapy, 30(4), 303-308.

https://doi.org/10.1097/PEP.0000000000000521

Sandlund, M., Lindh Waterworth, E., \& Häger, C. (2011). Using motion interactive games to promote physical activity and enhance motor performance in children with cerebral palsy. Developmental Neurorehabilitation, 14(1), 15-21.

https://doi.org/10.3109/17518423.2010.533329

Gatica-Rojas, V., Cartes-Velásquez, R., Méndez-Rebolledo, G., Olave-Godoy, F., \& Villalobos-Rebolledo, D. (2016). Change in functional balance after an exercise program with Nintendo Wii in Latino patients with cerebral palsy: a case series. Journal of Physical Therapy Science, 28(8), 2414-2417. https://doi.org/10.1589/jpts.28.2414

Gatica-Rojas, V., Méndez-Rebolledo, G., Guzman-Muñoz, E., Soto-Poblete, A., CartesVelásquez, R., Elgueta-Cancino, E., \& Cofré Lizama, L. E. (2017). Does Nintendo Wii Balance Board improve standing balance? A randomized controlled trial in children with cerebral palsy. European journal of physical and rehabilitation medicine, 53(4), 535-544. https://doi.org/10.23736/S1973-9087.16.04447-6 
Gatica-Rojas, V., Cartes-Velásquez, R., Méndez-Rebolledo, G., Guzman-Muñoz, E., \& Lizama, L. E. C. (2017). Effects of a Nintendo Wii exercise program on spasticity and static standing balance in spastic cerebral palsy. Developmental Neurorehabilitation, 20(6), 388391. https://doi.org/10.1080/17518423.2016.1211770

AlSaif, A. A., \& Alsenany, S. (2015). Effects of interactive games on motor performance in children with spastic cerebral palsy. Journal of Physical Therapy Science, 27(6), 2001-2003. https://doi.org/10.1589/jpts.27.2001

Sajan, J. E., John, J. A., Grace, P., Sabu, S. S., \& Tharion, G. (2017). Wii-based interactive video games as a supplement to conventional therapy for rehabilitation of children with cerebral palsy: A pilot, randomized controlled trial. Developmental Neurorehabilitation, 20(6), 361-367. https://doi.org/10.1080/17518423.2016.1252970

Sharan, D., Ajeesh, P. S., Rameshkumar, R., Mathankumar, M., Paulina, R. J., \& Manjula, M. (2012). Virtual reality based therapy for post operative rehabilitation of children with cerebral palsy. Work (Reading, Mass.), 41 Suppl 1, 3612-3615.

https://doi.org/10.3233/WOR-2012-0667-3612

Deutsch, J. E., Borbely, M., Filler, J., Huhn, K., \& Guarrera-Bowlby, P. (2008). Use of a Low-Cost, Commercially Available Gaming Console (Wii) for Rehabilitation of an Adolescent With Cerebral Palsy. Physical Therapy, 88(10), 1196-1207. https://doi.org/10.2522/ptj.20080062 
Tarakci, D., Ersoz Huseyinsinoglu, B., Tarakci, E., \& Razak Ozdincler, A. (2016). Effects of Nintendo Wii-Fit@ video games on balance in children with mild cerebral palsy. Pediatrics International, 58(10), 1042-1050. https://doi.org/10.1111/ped.12942

Jung, S.-H., Song, S.-H., Kim, S.-D., Lee, K., \& Lee, G.-C. (2018). Does virtual reality training using the Xbox Kinect have a positive effect on physical functioning in children with spastic cerebral palsy? A case series. Journal of Pediatric Rehabilitation Medicine, 11(2), 95101. https://doi.org/10.3233/PRM-160415

Cheung, J., Maron, M., Tatla, S., \& Jarus, T. (2013). Virtual reality as balance rehabilitation for children with brain injury: A case study. Technology and Disability, 25(3), 207-219. https://doi.org/10.3233/TAD-130383

Ayed, I., Ghazel, A., Jaume-i-Capó, A., Moya-Alcover, G., Varona, J., \& Martínez-Bueso, P. (2018). Feasibility of Kinect-Based Games for Balance Rehabilitation: A Case Study. Journal of Healthcare Engineering, 2018, 7574860-7574868. https://doi.org/10.1155/2018/7574860

Chanpimol, S., Seamon, B., Hernandez, H., Harris-Love, M., \& Blackman, M. R. (2017). Using Xbox kinect motion capture technology to improve clinical rehabilitation outcomes for balance and cardiovascular health in an individual with chronic TBI. Archives of Physiotherapy, 7(1), 1-11. https://doi.org/10.1186/s40945-017-0033-9 
Luna-Oliva, L., Ortiz-Gutiérrez, R. M., Cano-de la Cuerda, R., Piédrola, R. M., AlguacilDiego, I. M., Sánchez-Camarero, C., \& Martínez Culebras, M. D. C. (2013). Kinect Xbox 360 as a therapeutic modality for children with cerebral palsy in a school environment: a preliminary study. NeuroRehabilitation (Reading, Mass.), 33(4), 513-521. https://doi.org/10.3233/NRE-131001

Camara Machado, F. R., Antunes, P. P., Souza, J. D. M., Santos, A. C. D., Levandowski, D. C., \& Oliveira, A. A. D. (2017). Motor Improvement Using Motion Sensing Game Devices for Cerebral Palsy Rehabilitation. Journal of Motor Behavior, 49(3), 273-280. https://doi.org/10.1080/00222895.2016.1191422

Grigoriu, A. I., Lempereur, M., Bouvier, S., Padure, L., \& Brochard, S. (2021). Characteristics of newly acquired gait in toddlers with unilateral cerebral palsy: Implications for early rehabilitation. Annals of Physical and Rehabilitation Medicine, 64(3), 101333101333. https://doi.org/10.1016/j.rehab.2019.10.005

Bonnechère, B., Omelina, L., Jansen, B., \& Van Sint Jan, S. (2017). Balance improvement after physical therapy training using specially developed serious games for cerebral palsy children: preliminary results. Disability and Rehabilitation, 39(4), 403-406. https://doi.org/10.3109/09638288.2015.1073373

Leland, A., Tavakol, K., Scholten, J., Mathis, D., Maron, D., \& Bakhshi, S. (2017). The Role of Dual Tasking in the Assessment of Gait, Cognition and Community Reintegration of 
Veterans with Mild Traumatic Brain Injury. Materia Socio-Medica, 29(4), 251-256. https://doi.org/10.5455/msm.2017.29.251-256

Szturm, T., Sakhalkar, V., Boreskie, S., Marotta, J. J., Wu, C., \& Kanitkar, A. (2014). Integrated testing of standing balance and cognition: Test-retest reliability and construct validity. Gait \& Posture, 41(1), 146-152. https://doi.org/10.1016/j.gaitpost.2014.09.023

Bhatt, M. (2018). Evaluation of a computer game based rehabilitation system for assessment of balance and gait impairments in individuals with Parkinson's disease.

Szturm, T. J., Sakhalkar, V. S., Kanitkar, A., \& Nankar, M. (2017). Computerized Dual-Task Testing of Gait and Visuospatial Cognitive Functions; Test-Retest Reliability and Validity. Frontiers in Human Neuroscience, 11, 105-105. https://doi.org/10.3389/fnhum.2017.00105

Nankar, M., Szturm, T., Marotta, J., Shay, B., Beauchet, O., \& Allali, G. (2017). The interacting effects of treadmill walking and different types of visuospatial cognitive task: Discriminating dual task and age effects. Archives of Gerontology and Geriatrics, 73, 50-59. https://doi.org/10.1016/j.archger.2017.07.013

Deutsch, J. E., \& Westcott McCoy, S. (2017). Virtual Reality and Serious Games in Neurorehabilitation of Children and Adults: Prevention, Plasticity, and Participation. 
Pediatric Physical Therapy, 29 Suppl 3(Suppl 3 IV STEP 2016 CONFERENCE

PROCEEDINGS), S23-S36. https://doi.org/10.1097/PEP.0000000000000387

Sincero, S. M. (May 10, 2011). Operant Conditioning. Retrieved from

Explorable.com: https://explorable.com/operant-conditioning

Szturm, T., Betker, A. L., Moussavi, Z., Desai, A., \& Goodman, V. (2011). Effects of an Interactive Computer Game Exercise Regimen on Balance Impairment in Frail CommunityDwelling Older Adults: A Randomized Controlled Trial. Physical Therapy, 91(10), 1449_1462. https://doi.org/10.2522/ptj.20090205

Betker, A. L., Szturm, T., Moussavi, Z. K., \& Nett, C. (2006). Video Game-Based Exercises for Balance Rehabilitation: A Single-Subject Design. Archives of Physical Medicine and Rehabilitation, 87(8), 1141-1149. https://doi.org/10.1016/j.apmr.2006.04.010

Betker, A. L., Desai, A., Nett, C., Kapadia, N., \& Szturm, T. (2007). Game-based Exercises for Dynamic Short-Sitting Balance Rehabilitation of People With Chronic Spinal Cord and Traumatic Brain Injuries. Physical Therapy, 87(10), 1389-1398.

https://doi.org/10.2522/ptj.20060229

Kanitkar, A., Szturm, T., Parmar, S., Gandhi, D. B., Rempel, G. R., Restall, G., Sharma, M., Narayan, A., Pandian, J., Naik, N., Savadatti, R. R., \& Kamate, M. A. (2017). The Effectiveness of a Computer Game-Based Rehabilitation Platform for Children With 
Cerebral Palsy: Protocol for a Randomized Clinical Trial. JMIR Research Protocols, 6(5), e93-e93. https://doi.org/10.2196/resprot.6846

Mansournia, M. A., Higgins, J. P. T., Sterne, J. A. C., \& Hernán, M. A. (2017). Biases in Randomized Trials: A Conversation Between Trialists and Epidemiologists. Epidemiology (Cambridge, Mass.), 28(1), 54-59. https://doi.org/10.1097/EDE.0000000000000564

Bodkin, A. W., Robinson, C., \& Perales, F. P. (2003). Reliability and validity of the gross motor function classification system for cerebral palsy. Pediatric Physical Therapy, 15(4), 247-252. https://doi.org/10.1097/01.PEP.0000096384.19136.02

Mutlu, A., Livanelioglu, A., \& Gunel, M. K. (2008). Reliability of Ashworth and Modified Ashworth scales in children with spastic cerebral palsy. BMC Musculoskeletal Disorders, 9(1), 44-44. https://doi.org/10.1186/1471-2474-9-44

Wang, H.-H., Liao, H.-F., \& Hsieh, C.-L. (2006). Reliability, Sensitivity to Change, and Responsiveness of the Peabody Developmental Motor Scales-Second Edition for Children With Cerebral Palsy. Physical Therapy, 86(10), 1351-1359. https://doi.org/10.2522/ptj.20050259

van Hartingsveldt, M. J., Cup, E. H., \& Oostendorp, R. A. (2005). Reliability and validity of the fine motor scale of the Peabody Developmental Motor Scales-2. Occupational Therapy International, 12(1), 1-13. https://doi.org/10.1002/oti.11 
Zanella, L. W., Valentini, N. C., Copetti, F., \& Nobre, G. C. (2021). Peabody Developmental Motor Scales - Second Edition (PDMS-2): Reliability, content and construct validity evidence for Brazilian children. Research in Developmental Disabilities, 111, 103871103871. https://doi.org/10.1016/j.ridd.2021.103871

Alotaibi, M., Long, T., Kennedy, E., \& Bavishi, S. (2014). The efficacy of GMFM-88 and GMFM-66 to detect changes in gross motor function in children with cerebral palsy (CP): a literature review. Disability and Rehabilitation, 36(8), 617-627. https://doi.org/10.3109/09638288.2013.805820

Wei, S., Su-Juan, W., Yuan-Gui, L., Hong, Y., Xiu-Juan, X., \& Xiao-Mei, S. (2006). Reliability and validity of the GMFM-66 in 0- to 3-year-old children with cerebral palsy. American Journal of Physical Medicine \& Rehabilitation, 85(2), 141-147. https://doi.org/10.1097/01.phm.0000197585.68302.25

Chen, C., Shen, I., Chen, C., Wu, C., Liu, W.-Y., \& Chung, C. (2013). Validity, responsiveness, minimal detectable change, and minimal clinically important change of Pediatric Balance Scale in children with cerebral palsy. Research in Developmental Disabilities, 34(3), 916-922. https://doi.org/10.1016/j.ridd.2012.11.006 
Thomas, D. R. (2006). A General Inductive Approach for Analyzing Qualitative Evaluation Data. The American Journal of Evaluation, 27(2), 237-246.

https://doi.org/10.1177/1098214005283748

Tickle-Degnen, L. (2013). Nuts and bolts of conducting feasibility studies. The American Journal of Occupational Therapy, 67(2), 171-176. https://doi.org/10.5014/ajot.2013.006270

Hadi, M. A., \& Closs, S. J. (2016). Applications of mixed-methods methodology in clinical pharmacy research. International Journal of Clinical Pharmacy, 38(3), 635-640. https://doi.org/10.1007/s11096-015-0231-z

Cope, D. G. (2015). Conducting pilot and feasibility studies. Oncology Nursing Forum, 42(2), 196-197. https://doi.org/10.1188/15.ONF.196-197

Almalki, S. (2016). Integrating Quantitative and Qualitative Data in Mixed Methods Research—Challenges and Benefits. Journal of Education and Learning, 5(3), 288-. https://doi.org/10.5539/jel.v5n3p288

Tsianakas, V., Harris, J., Ream, E., Van Hemelrijck, M., Purushotham, A., Mucci, L., Green, J. S. A., Fewster, J., \& Armes, J. (2017). CanWalk: a feasibility study with embedded randomised controlled trial pilot of a walking intervention for people with recurrent or 
metastatic cancer. BMJ Open, 7(2), e013719-e013719. https://doi.org/10.1136/bmjopen$\underline{2016-013719}$

Driscoll, D. L., Appiah-Yeboah, A., Salib, P., \& Rupert, D.J. (2007) Merging qualitative and quantitative data in mixed methods research?: How to and why not. Ecol Environ Anthropol 3(1):18-28. http://digitalcommons.unl.edu/icwdmeea/18

Malina, M. A., Nørreklit, H. S. ., \& Selto, F. H. (2011). Lessons learned: advantages and disadvantages of mixed method research. Qualitative Research in Accounting and Management, 8(1), 59-71. https://doi.org/10.1108/11766091111124702

Varpio, L., Ajjawi, R., Monrouxe, L. V., O’Brien, B. C., \& Rees, C. E. (2017). Shedding the cobra effect: problematising thematic emergence, triangulation, saturation and member checking. Medical Education, 51(1), 40-50. https://doi.org/10.1111/medu.13124

Oleinik, A. (2011). Mixing quantitative and qualitative content analysis: triangulation at work. Quality \& Quantity, 45(4), 859-873. https://doi.org/10.1007/s11135-010-9399-4

Donker, S. ., Ledebt, A., Roerdink, M., Savelsbergh, G. J. ., \& Beek, P. . (2008). Children with cerebral palsy exhibit greater and more regular postural sway than typically developing children. Experimental Brain Research, 184(3), 363-370. https://doi.org/10.1007/s00221$\underline{007-1105-y}$ 
Desai, A., Goodman, V., Kapadia, N., Shay, B. L., \& Szturm, T. (2010). Relationship Between Dynamic Balance Measures and Functional Performance in Community-Dwelling Elderly People. Physical Therapy, 90(5), 748-760. https://doi.org/10.2522/ptj.20090100

Dewar, R. M., Tucker, K., Claus, A. P., van den Hoorn, W., Ware, R. S., \& Johnston, L. M. (2021). Evaluating validity of the Kids-Balance Evaluation Systems Test (Kids-BESTest) Clinical Test of Sensory Integration of Balance (CTSIB) criteria to categorise stance postural control of ambulant children with CP. Disability and Rehabilitation, 1-8. https://doi.org/10.1080/09638288.2021.1887374

Oeffinger, D., Bagley, A., Rogers, S., Gorton, G., Kryscio, R., Abel, M., Damiano, D., Barnes, D., \& Tylkowski, C. (2008). Outcome tools used for ambulatory children with cerebral palsy: responsiveness and minimum clinically important differences. Developmental Medicine and Child Neurology, 50(12), 918-925. https://doi.org/10.1111/j.1469$\underline{8749.2008 .03150 . x}$

Reid, D. T. (2002). Benefits of a virtual play rehabilitation environment for children with cerebral palsy on perceptions of self-efficacy: a pilot study. Pediatric Rehabilitation, 5(3), 141-148. https://doi.org/10.1080/1363849021000039344 


\section{ELBADAWI IBRAHIM MOHAMMAD ELHINIDI, MARWA MOSTAFA IBRAHIM}

ISMAEEL, \& TAMER MOHAMED EL-SAEED. (2016). Effect of dual-task training on postural stability in children with infantile hemiparesis. Journal of physical therapy science, 28(3), 875-880. https://doi.org/10.1589/jpts.28.875

Roostaei, M., Raji, P., Morone, G., Razi, B., \& Khademi-Kalantari, K. (2021). The effect of dual-task conditions on gait and balance performance in children with cerebral palsy: A systematic review and meta-analysis of observational studies. Journal of Bodywork and Movement Therapies, 26, 448-462. https://doi.org/10.1016/j.jbmt.2020.12.011

Li, K. Z. ., Bherer, L., Mirelman, A., Maidan, I., \& Hausdorff, J. M. (2018). Cognitive involvement in balance, gait, and dual-tasking in aging: A focused review from the neuroscience of aging perspective. Frontiers in Neurology, 9, 913-913. https://doi.org/10.3389/fneur.2018.00913

Yu, H., Wang, Z., Liu, C., Dai, P., Lan, Y., \& Xu, G. (2021). Effect of Cognitive Function on Balance and Posture Control after Stroke. Neural Plasticity, 2021, 6636999-6636999. https://doi.org/10.1155/2021/6636999 
Kelders, S. M., Sommers-Spijkerman, M., \& Goldberg, J. (2018). Investigating the Direct Impact of a Gamified Versus Nongamified Well-Being Intervention: An Exploratory Experiment. Journal of Medical Internet Research, 20(7), e247-e247. https://doi.org/10.2196/jmir.9923

Mahana, B., Bhatt, M., Gupta, A., Hobson, D.E., Ko, J.H., \& Szturm, T. (2019). Does use of a dual task cognitive game based treadmill platform improve balance and gait in Parkinson Disease? A feasibility study. Brain, Body, Cognition 2019;9(4):593-605 https://hdl.handle.net/1993/34269

Kanitkar, A., Parmar, S. T., Szturm, T. J., Restall, G., Rempel, G., \& Sepehri, N. (2021). Parents' Perspectives on a Computer Game-Assisted Rehabilitation Program for Manual Dexterity in Children With Cerebral Palsy: Qualitative Analysis of Expectations, Child Engagement, and Benefits. JMIR Rehabilitation and Assistive Technologies, 8(2), e24337e24337. https://doi.org/10.2196/24337

Jain, M., \& Passi, G. R. (2005). Assessment of a modified Mini-Mental Scale for cognitive functions in children. Indian Pediatrics, 42(9), 907-912. https://search.lib.umanitoba.ca/permalink/01UMB_INST/k6qbb2/cdi_proquest_miscellaneou $\underline{\text { s_68661627 }}$ 
Kang, M., Smith, E., Goldsmith, C. H., Switzer, L., Rosenbaum, P., Wright, F. V., \&

Fehlings, D. (2020). Documenting change with the Canadian Occupational Performance Measure for children with cerebral palsy. Developmental Medicine and Child Neurology, 62(10), 1154-1160. https://doi.org/10.1111/dmcn.14569 